Prepared in cooperation with the Pennsylvania Department of Environmental Protection

\title{
Selected Streamflow Statistics for Streamgage Locations in and near Pennsylvania
}

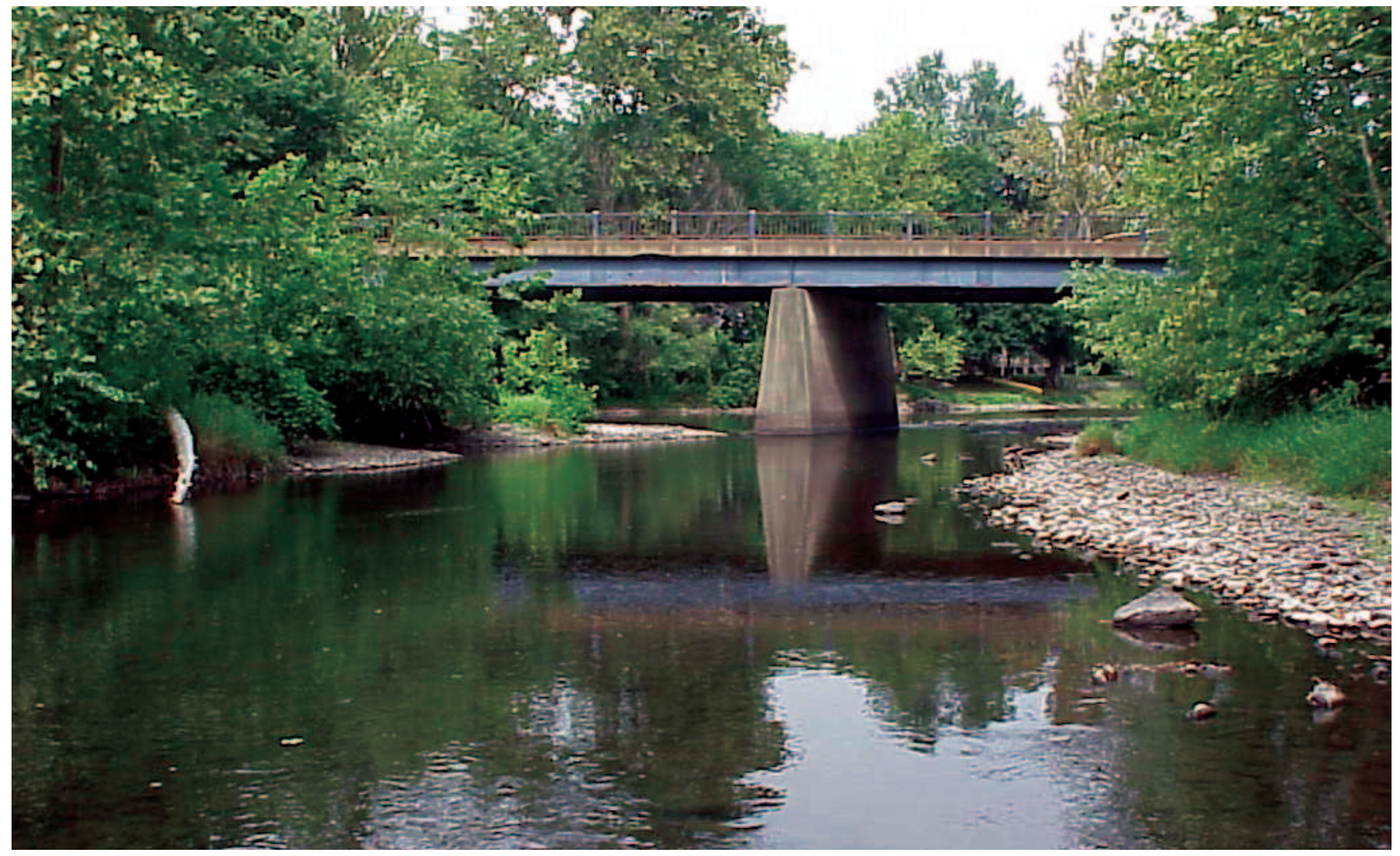

Open-File Report 2011-1070

U.S. Department of the Interior

U.S. Geological Survey 
Cover. Tunkhannock Creek and Highway 6 overpass downstream from U.S. Geological Survey streamgage 01534000 Tunkhannock Creek near Tunkhannock, PA. (Photo by Andrew Reif, USGS) 


\section{Selected Streamflow Statistics for Streamgage Locations in and near Pennsylvania}

By Marla H. Stuckey and Mark A. Roland

Prepared in cooperation with the

Pennsylvania Department of Environmental Protection

Open-File Report 2011-1070 


\title{
U.S. Department of the Interior \\ KEN SALAZAR, Secretary \\ U.S. Geological Survey \\ Marcia K. McNutt, Director
}

\author{
U.S. Geological Survey, Reston, Virginia: 2011
}

For more information on the USGS - the Federal source for science about the Earth, its natural and living resources, natural hazards, and the environment, visit http://www.usgs.gov or call 1-888-ASK-USGS.

For an overview of USGS information products, including maps, imagery, and publications, visit http://www.usgs.gov/pubprod

To order this and other USGS information products, visit http://store.usgs.gov

Any use of trade, product, or firm names is for descriptive purposes only and does not imply endorsement by the U.S. Government.

Although this report is in the public domain, permission must be secured from the individual copyright owners to reproduce any copyrighted materials contained within this report.

Suggested citation:

Stuckey, M.H., and Roland, M.A., 2011, Selected streamflow statistics for streamgage locations in and near Pennsylvania: U.S. Geological Survey Scientific Investigations Report 2011-1070, 88 p. 


\section{Contents}

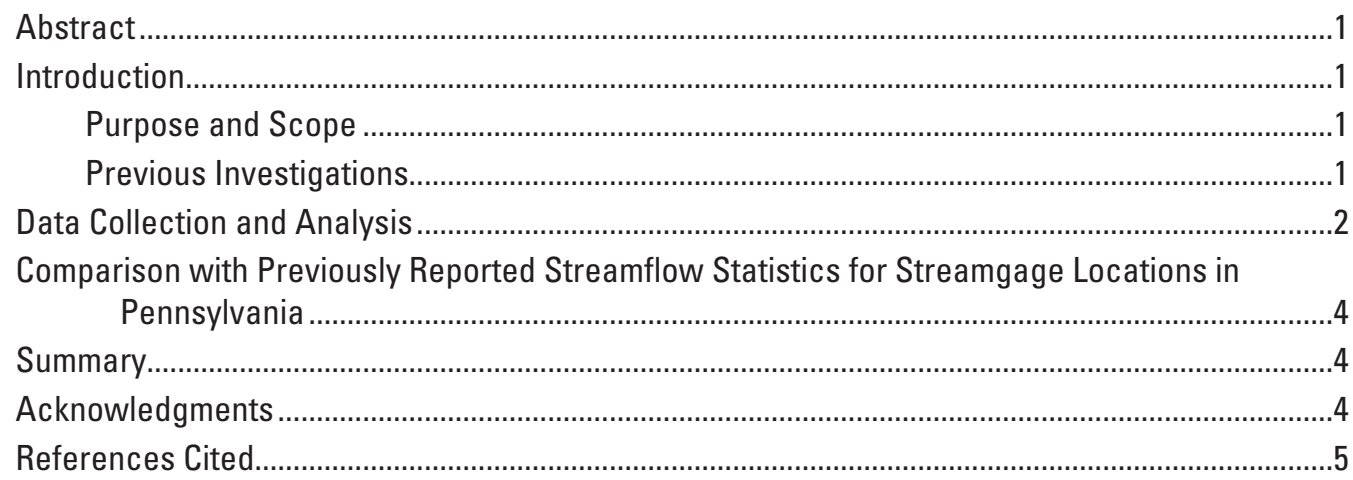

\section{Figures}

1. Map showing location of U.S. Geological Survey streamgage locations with updated streamflow statistics

\section{Tables}

1. List of U.S. Geological Survey streamgage locations in and near Pennsylvania with updated streamflow statistics ............................................................................

2. Selected low-flow statistics for streamgage locations in and near Pennsylvania .........20

3. Selected base-flow statistics for streamgage locations in and near Pennsylvania.......34

4. Selected flood-flow statistics for streamgage locations in and near Pennsylvania .......48

5. Selected mean-flow and flow-duration statistics for streamgage locations in and near Pennsylvania ........................................................................................60

6. Comparison of streamflow statistics for 72 streamgage locations in Pennsylvania to previously reported streamflow statistics ..................................................................88 


\section{Conversion Factors}

\begin{tabular}{lcl}
\multicolumn{1}{c}{ Inch/Pound to SI } & \multicolumn{1}{c}{ By } & \multicolumn{1}{c}{ To obtain } \\
\hline \multicolumn{1}{c}{ Multiply } & Area & \\
\hline $\begin{array}{l}\text { square mile }\left(\mathrm{mi}^{2}\right) \\
\text { square mile }\left(\mathrm{mi}^{2}\right)\end{array}$ & 259.0 & hectare $($ ha) \\
& 2.590 & square kilometer $\left(\mathrm{km}^{2}\right)$ \\
\hline \multicolumn{2}{c}{ Flow rate } & \\
\hline $\begin{array}{l}\text { foot per second }(\mathrm{ft} / \mathrm{s}) \\
\text { cubic foot per second }(\mathrm{ft} / \mathrm{s})\end{array}$ & 0.3048 & meter per second $(\mathrm{m} / \mathrm{s})$ \\
cubic foot per second per square & 0.02832 & cubic meter per second $\left(\mathrm{m}^{3} / \mathrm{s}\right)$ \\
mile $\left[\left(\mathrm{ft}^{3} / \mathrm{s}\right) / \mathrm{mi}^{2}\right]$ & 0.01093 & cubic meter per second per square \\
& & kilometer $\left[\left(\mathrm{m}^{3} / \mathrm{s}\right) / \mathrm{km}^{2}\right]$ \\
\hline
\end{tabular}

Vertical coordinate information is referenced to the North American Vertical Datum of 1988 (NAVD 88).

Horizontal coordinate information is referenced to the North American Datum of 1983 (NAD 83). 


\title{
Selected Streamflow Statistics for Streamgage Locations in and near Pennsylvania
}

\author{
By Marla H. Stuckey and Mark A. Roland
}

\section{Abstract}

Streamflow statistics are used routinely by watershed planners, regulators, engineers, and managers for waterresource projects. Streamflow statistics for a streamgage can change over time as more flow data become available and (or) land use or climate in the watershed changes; consequently, streamflow statistics should be updated periodically. This report, done in cooperation with the Pennsylvania Department of Environmental Protection, presents updated streamflow statistics for 526 U.S. Geological Survey (USGS) continuous-record streamgages in Pennsylvania, New York, New Jersey, Delaware, Maryland, West Virginia, and Ohio using data collected through 2008. The following statistics are presented: low flows (1-day, 10-year; 7-day, 10-year; 7-day, 2-year; 30-day, 10-year; 30-day, 2-year; and 90-day, 10-year low flows), base flows (10-year, 25-year, and 50-year base flows), peak flows (50-, 20-, 10-, 4-, 2-, 1-, and 0.2-percent annual exceedance flood), mean flows (mean annual flow and harmonic mean flow), and flow durations (99-, 95-, 90-, 85-, 80-, 70-, 60-, 50-, 40-, 30-, 20-, 15-, 10-, 5-, and 1-percent exceedance). Streamflow statistics (7-day, 10-year and 30-day, 10-year low flows; harmonic mean; and 95-, 50-, and 5-percent flow duration exceedances) for 72 streamgage locations in Pennsylvania were compared using a Wilcoxon signed-rank test to previously reported statistics. A statistically significant difference was found between the data sets for the 30-day, 10-year low-flow; harmonic mean; 50-percent flow-duration exceedance; and 5-percent flow-duration exceedance statistics. No significant difference was found between the data sets for the 7-day, 10-year low-flow and 95-percent flowduration exceedance statistics. The lower flow statistics show a decrease since 1996, and the mid- to higher flows show an increase since 1996.

\section{Introduction}

Streamflow statistics are used routinely by watershed planners, regulators, engineers, and managers for waterresource projects. Low-flow statistics are used to determine water availability, water-use allocations, assimilative capacities, and aquatic-habitat needs. Flood-flow statistics are used for Flood Insurance Studies (FIS), flood-plain management, and the design of bridges and flood-control structures. Streamflow statistics for a streamgage can change over time as more data become available or as land use or climate in the watershed changes, so streamflow statistics should be updated periodically.

\section{Purpose and Scope}

This report presents updated streamflow statistics for 526 U.S. Geological Survey (USGS) continuous-record streamgage locations in and near Pennsylvania using data collected through the 2008 water yeart. Streamgages from Pennsylvania (306), New York (65), New Jersey (28), Delaware (13), Maryland (68), West Virginia (21), and Ohio (25) were included in this analysis. The following statistics are presented: low flows (1-day, 10-year; 7-day, 10-year; 7-day, 2-year; 30-day, 10-year; 30-day, 2-year; and 90-day, 10-year low flows), base flows (10-year, 25-year, and 50-year base flows), peak flows (50-, 20-, 10-, 4-, 2-, 1-, and 0.2-percent annual exceedance flood), mean flows (mean annual flow and harmonic mean flow), and flow durations (99-, 95-, 90-, 85-, 80-, 70-, 60-, 50-, 40-, 30-, 20-, 15-, 10-, 5-, and 1- percent exceedance). This development of updated streamflow statistics was done in cooperation with the Pennsylvania Department of Environmental Protection (PaDEP). The selection of streamgage locations was determined by the need from PaDEP for updated streamflow information at reference sites to assist in permit application review. Streamflow statistics for streamgages in Pennsylvania will be displayed on the webbased application Pennsylvania StreamStats (Stuckey and Hoffman, 2010).

\section{Previous Investigations}

Streamflow statistics for USGS streamgage locations in Pennsylvania have been published in many USGS reports. Stuckey (2006) developed low-flow, base-flow, and mean-flow

\footnotetext{
${ }^{1}$ Water year is defined as a 12-month period beginning October 1 and ending September 30. The water year is designated by the calendar year in which it ends.
} 
regression equations and reported statistics for 293 continuous-record and partial-record streamgages using data through the 2002 climatic year ${ }^{2}$. Roland and Stuckey (2008) developed flood-flow regression equations and reported flood-flow statistics for 306 continuous-record and crest-stage streamgages using data through the 2005 or 2006 water year. Low-flow and flood-flow statistics for additional streamgages not included in the previously mentioned reports were published by Ehlke and Reed (1999). Flow durations were published by Page and Shaw (1977) for continuous-record and miscellaneous streamgages with sufficient data through the 1972 water year, and Wolock (2003) computed streamflow statistics, including flow durations, for the conterminous United States. Streamflow statistics for Pennsylvania streamgages also may be found in USGS reports published from surrounding states. Guidance on techniques regarding weighting and transference of flood-flow statistics is described by Stuckey and Reed (2000) and for low-flow statistics in documentation found at the USGS-PA Low-Flow Statistics web application (http://paapps.er.usgs.gov/flowstats/).

Similar reports have also been published by surrounding states and present streamflow statistics for gaged locations. Peak-flow statistics for streamgage locations in New York are presented by Lumia and others (2006) and also by Suro and others (2009). Flood magnitude and frequencies were published by Schopp and Firda (2008) for the streamgages on the Delaware River mainstem. Watson and Schopp (2009) presented peak-flow statistics for streamgage locations in New Jersey using data through the 2007 water year. Peakflow statistics for Delaware streamgages were published in 2006 by Ries and Dillow. Peak-flow statistics for Maryland streamgage locations were presented by Dillow (1995). Doheny and Banks (2010) presented low-flow statistics and Ries (2006) presented low-flow and flow-duration statistics for Maryland streamgages. Low-flow and flow-duration statistics for streamgage locations in West Virginia were computed by Wiley (2006) using a common period of record, and peak-flow statistics were presented by Wiley and Atkins (2010). Koltun (2003) presented flood-peak discharges for rural, unregulated streamgages in Ohio.

\section{Data Collection and Analysis}

Streamflow data collected through the 2008 water year for 526 continuous-record streamgages in and near Pennsylvania with a minimum of 9 years of data were selected for this analysis (fig. 1; table 1, at end of report). Streamgages with periods of record affected by upstream regulation were split into pre-regulation and post-regulation periods. Streamflow statistics were determined for both the pre- and

\footnotetext{
${ }^{2}$ Climatic year is defined as 12-month period beginning April 1 and ending March 31. The climatic year is designated by the calendar year in which it ends.
}

post-regulation periods, when the period had at least 9 years of data. Periods of regulation were determined from USGS peakflow files, indicating known regulation of flow, previously published USGS reports, and engineering judgment. Classification of known regulation as noted in the peak flow files generally was used if upstream reservoirs controlled more than 10 percent of the drainage area at the streamgage. Regulation is identified for each streamgage in table 1.

After the appropriate period of record was determined for each streamgage, daily flow values were retrieved from the USGS National Water Information System Web (NWISWeb) (U.S. Geological Survey, 2002) using software described by Granato (2009). A limitation to the version of the software used is that data prior to the year 1900 can not be retrieved. Therefore, data prior to 1900 for two streamgages were not used in the analysis. The files were reviewed for completeness and accuracy and entered into a watershed data management (WDM) file (Lumb and others, 1990).

Peak-flow data for each streamgage were retrieved from NWISWeb using the batch download option, excluding partial peaks. The retrieved peak-flow files contain one peak per year for the systematic record and any peaks recorded outside the systematic record, termed historical peaks. Peak flows recorded on a stream with regulated flow and any historical peaks are identified with codes in the file. The peak-flow files were reviewed for accuracy and completeness, and regulation and historical peak codes were noted. Historical peaks generally were included unless the peak was questionable or was not believed to have been the largest peak during the historical period. Streamgages unaffected by upstream regulation that were included in the recent flood-flow regression analysis for Pennsylvania (Roland and Stuckey, 2008) were not updated because of minimal increase of data. Flood-flow statistics were also not updated for streamgages in New York, New Jersey, Delaware, and West Virginia because of recent publications and revised regional skews used in those states.

Utilizing the WDM file, daily flow values from stream data recorded at each streamgage were entered into the USGS Surface Water Statistics (SWSTAT) software to calculate low-flow n-day, T-year statistics, flow durations, and the mean annual flow. To determine the base-flow statistics, the daily flow values from each streamgage were first separated into base-flow and surface-runoff components using the local-minimum method in the hydrograph separation computer program HYSEP (Sloto and Crouse, 1996). HYSEP limits the number of years of record to 100 years; 19 streamgages had records longer than 100 years, but only data from the most recent 100 years were used in the analysis. The adjusted daily flow value datasets were then entered into SWSTAT to calculate the base-flow statistics. Flood-flow statistics were determined using the USGS Peak Flow Frequency (PKFQWin) software for Windows (Flynn and others, 2006). Harmonic mean was determined using the U.S. Environmental Protection Agency DFLOW software (http://www.epa.gov/waterscience/models/ dflow/) utilizing file-preparation software developed by Granato (2009). 


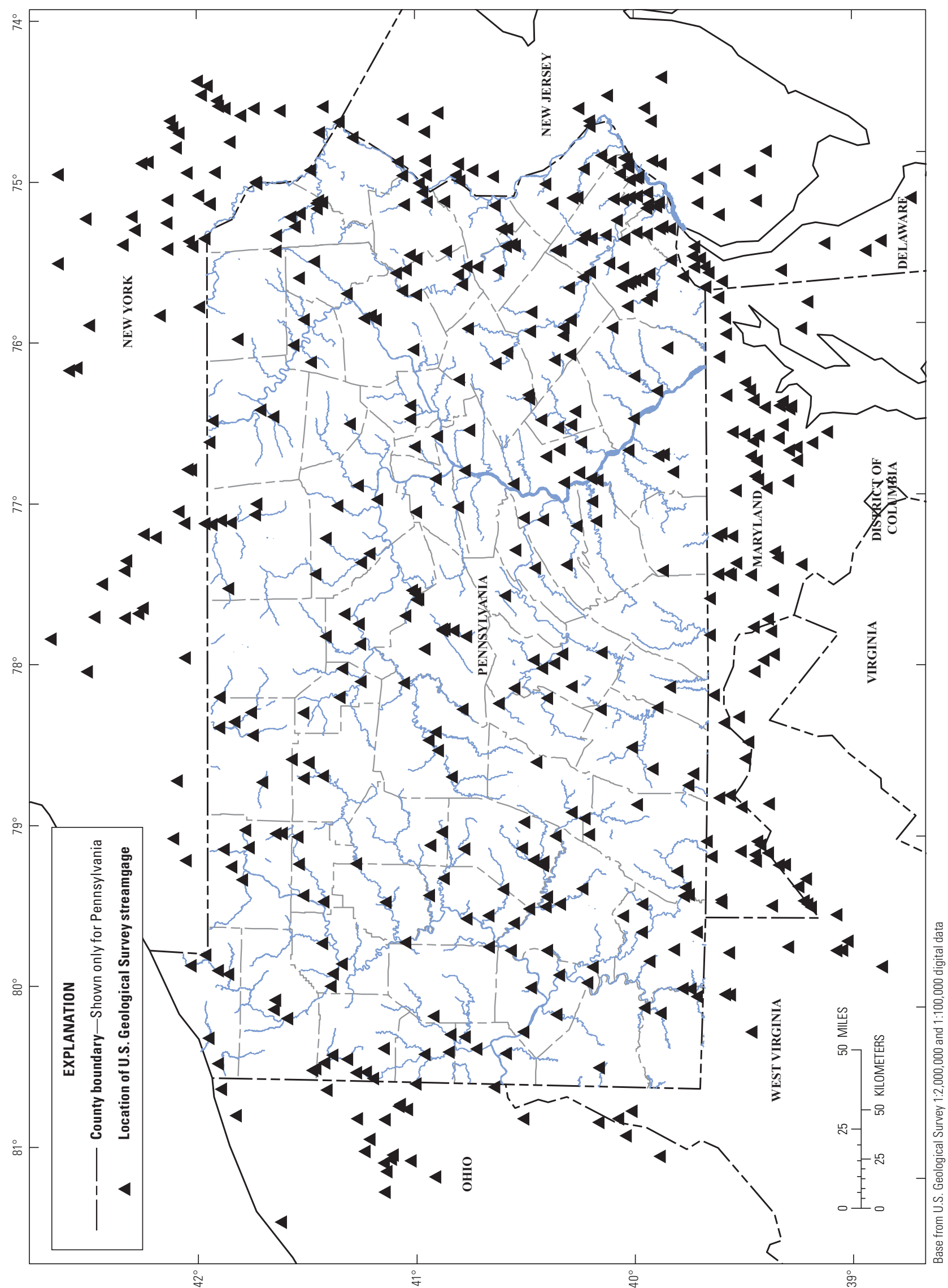


The USGS software described above is publicly available on the web at http://water.usgs.gov/osw/methods.html. Standard options available in these programs were used to generate the statistics. Low-flow statistics were determined using methods described by Riggs (1972) and Matalas (1963). Flood-flow statistics were determined using methods described by the Water Resources Council, Hydrology Committee (1981) and implemented in the PKFQWin software. The weighted skew option within PKFQWin was used to determine the flood-flow frequencies for unregulated streams; the station skew was used to determine flood-flow frequencies for regulated streams. The Log-Pearson Type III frequency distribution was fit to the data using method of moments (mean, standard deviation, and coefficient of skewness) with adjustments for the flood flows for low outliers, high outliers, historic peaks, and generalized skew.

The resultant streamflow statistics for the 526 streamgages are listed in tables $2-5$ (at end of report). If the period of record is split for a streamgage because of regulation, the streamgage is listed more than once in the table. The individual period of record used to compute the different statistics for the same streamgage may differ slightly depending on whether or not historical peak(s) were included in the computation, the type of year (climatic or water) used to compute the statistic, or regulation occurring at different flow regimes.

\section{Comparison with Previously Reported Streamflow Statistics for Streamgage Locations in Pennsylvania}

The streamflow statistics generated from this analysis were compared to previously reported streamflow statistics to determine if there is a significant change and whether the statistics have increased or decreased. Seventy-two current continuous-record streamgages with data through the 2008 water year and unregulated flow were selected for this comparison. The 7-day, 10-year low flow (7Q10), 30-day, 10-year low flow (30Q10), harmonic mean, and 95-, 50-, and 5-percent flow-duration exceedance (P95, P50, and P5, respectively) for the 72 streamgages were compared to flow statistics published by Ehlke and Reed (1999), which used streamflow data through the 1995 or 1996 climatic year. Flood-flow statistics were excluded from this comparison because most of the 72 streamgages were not updated as part of the current analysis.

A Wilcoxon signed-rank test was used to determine if there was a significant change in the median value of the six flow statistics from 1996 to 2008. (Helsel and Hirsch, 1993). The analysis was done using a 95-percent confidence level $($ alpha $=0.05$ ). The change in median streamflow statistics since 1996 and the results of the Wilcoxon signed-rank test are shown in table 6 (at end of report). This change in streamflow characteristic was determined from the median difference between the data sets and is depicted as an increase or decrease in the flow characteristic. A significant difference was found between the data sets for the $30 \mathrm{Q} 10$, harmonic mean, P50, and P5 statistics; no significant difference was found between the data sets for the 7Q10 and P95 statistics. The lower flow statistics (7Q10, 30Q10, and P95) show a decrease since 1996, and the mid- to higher flows (harmonic mean, P50, and P5) show an increase since 1996. This can be partly attributed to the drought conditions experienced by much of Pennsylvania in the late 1990s and early 2000s and the large flood events of 1999, 2004, and 2006.

\section{Summary}

Streamflow statistics are used routinely by water-resource professionals for a variety of purposes, including water-use allocations, aquatic-habitat needs, flood-plain management, and the design of bridges and flood-control structures. Streamflow statistics for a streamgage can change over time as more data become available and (or) as land use or climate in the watershed changes; consequently, streamflow statistics should be updated periodically. In cooperation with the Pennsylvania Department of Environmental Protection, this report presents updated streamflow statistics for 526 U.S. Geological Survey (USGS) continuous-record streamgages in Pennsylvania, New York, New Jersey, Delaware, Maryland, West Virginia, and Ohio. The following streamflow statistics were updated: low flows (1-day, 10-year; 7-day, 10-year; 7-day, 2-year; 30-day, 10-year; 30-day, 2-year; and 90-day, 10-year low flows), base flows (10-year, 25 -year, and 50-year base flows), peak flows (50-, 20-, 10-, 4-, 2-, 1-, and 0.2-percent annual exceedance flood), mean flows (mean annual flow and harmonic mean flow), and flow durations (99-, 95-, 90-, 85-, 80-, 70-, 60-, 50-, 40-, 30-, 20-, 15-, 10-, 5-, and 1-percent exceedance).

Streamflow statistics (7-day, 10-year and 30-day, 10-year low flows; harmonic mean; and 95-, 50-, and 5-percent flow duration exceedances) for 72 streamgage locations in Pennsylvania were compared using a Wilcoxon signed-rank test to previously reported statistics. A significant difference was found between the data sets for the 30-day, 10-year; harmonic mean; 50-percent flow-duration exceedance; and 5-percent flow-duration exceedance statistics; no significant difference was found between the data sets for the 7-day, 10-year lowflow and 95-percent flow-duration exceedance statistics. The lower flow statistics show a decrease since 1996, and the midto higher flows show an increase since 1996.

\section{Acknowledgments}

Special thanks are extended to the Pennsylvania Water Science Center (and New York, New Jersey, Delaware, Maryland, West Virginia, and Ohio) Hydrologic Surveillance Program staffs for their compilation and meticulous review 
of streamgage data used to determine the streamflow statistics. Thanks also to Gregory Granato (Massachusetts Water Science Center) for developing software that assisted with data preparation, Edward Koerkle (Pennsylvania Water Science Center) for script development and statistical expertise, and Scott Hoffman (Pennsylvania Water Science Center) for GIS assistance. Clinton Hittle and Peter Cinotto of the USGS provided technical reviews of the manuscript. Gary Firda, Ed Doheny, Jeff Wiley, and Greg Koltun of the USGS provided review of statistics for streamgages in New York, Maryland and Delaware, West Virginia, and Ohio, respectively.

\section{References Cited}

Doheny, E.J., and Banks, W.S.L., 2010, Selected low-flow frequency statistics for continuous-record streamgage locations in Maryland, 2010: U.S. Geological Survey Open-File Report 2010-1310, 22 p.

Dillow, J.A., 1995, Technique for estimating magnitude and frequency of peak flows in Maryland: U.S. Geological Survey Water Resources Investigations Report 95-4154, 55 p.

Ehlke, M.H., and Reed, L.A., 1999, Comparison of methods for computing streamflow statistics for Pennsylvania streams: U.S. Geological Survey Water-Resources Investigations Report 99-4068, 80 p.

Flynn, K.M., Kirby, W.H., Mason, R.R., and Cohn, T.A., 2006, Estimating magnitude and frequency of floods using the PeakFQ program: U.S. Geological Survey Fact Sheet 2006-3143, 2 p.

Granato, G.E., 2009, Computer programs for obtaining and analyzing daily mean streamflow data from the U.S. Geological Survey National Water Information System Web Site: U.S. Geological Survey Open-File Report 2008-1362, 123 p. on CD-ROM, 5 appendixes.

Helsel, D.R., and Hirsch, R.M., 1993, Statistical methods in water resources: Amsterdam, The Netherlands, Elsevier, Sarah Burgerhartstraat 25, 1000 AE Studies in Environmental Science 49, 529 p.

Koltun, G.F., 2003, Techniques for estimating flood-peak discharges of rural, unregulated streams in Ohio: U.S. Geological Survey Water-Resources Investigations Report 03-4164, $76 \mathrm{p}$.

Lumb, A.M., Kittle, J.L., Jr., and Flynn, K.M., 1990, Users manual for ANNIE, a computer program for interactive hydrologic analyses and data management: U.S. Geological Survey Water-Resources Investigations Report 89-4080, $236 \mathrm{p}$.
Lumia, Richard, Freehafer, D.A., and Smith, M.J., 2006, Magnitude and frequency of floods in New York: U.S. Geological Survey Scientific Investigations Report 2006-5112, $152 \mathrm{p}$.

Matalas, N.C., 1963, Probability distribution of low flows: U.S. Geological Survey Professional Paper 434-A, 27 p.

Page, L.V., and Shaw, L.C., 1977, Low-flow statistics of Pennsylvania streams: Pennsylvania Department of Environmental Resources, Water Resources Bulletin No. 12, 441 p.

Riggs, H.C., 1972, Low-flow investigations: U.S. Geological Survey Techniques of Water-Resources Investigations, book 4, chap. B1, 18 p.

Ries, K.G., III, and Dillow, J.J.A., 2006, Magnitude and frequency of floods on nontidal streams in Delaware: U.S. Geological Survey Scientific Investigations Report 20065146, 59 p.

Roland, M.A., and Stuckey, M.H., 2008, Regression equations for estimating magnitude and frequency of peak flows for ungaged streams in Pennsylvania: U.S. Geological Survey Scientific Investigations Report 2008-5102, 76 p.

Schopp, R.D., and Firda, G.D., 2008, Flood magnitude and frequency of the Delaware River in New Jersey, New York, and Pennsylvania: U.S. Geological Survey Open-File Report 2008-1203, 7 p.

Sloto, R.A., and Crouse, M.Y., 1996, HYSEP-A computer program for streamflow hydrograph separation and analysis: U.S. Geological Survey Water-Resources Investigations Report 96-4040, 46 p.

Stuckey, M.H., and Hoffman, S.A., 2010, Pennsylvania StreamStats: A web-based application for obtaining waterresource-related information: U.S. Geological Survey Fact Sheet 2010-3086, 2 p.

Stuckey, M.H., 2006, Low-flow, base-flow, and mean-flow regression equations for Pennsylvania streams: U.S. Geological Survey Scientific Investigations Report 2006-5130, $84 \mathrm{p}$.

Stuckey, M.H., and Reed, L.A., 2000, Techniques for estimating magnitude and frequency of peak flows for Pennsylvania streams: U.S. Geological Survey Water-Resources Investigations Report 00-4189, 43 p.

Suro, T.P., Firda, G.D., and Szabo, C.O., 2009, Flood of June 26-29, 2006, Mohawk, Delaware, and Susquehanna River Basins, New York: U.S. Geological Survey Open-File Report 2009-1063, 354 p.

U.S. Geological Survey, 2002, NWISWeb-New site for the Nation's water data: U.S. Geological Survey Fact Sheet 128-02, 2 p. 
Water Resources Council, Hydrology Committee, 1981, Guidelines for determining flood flow frequencies: Washington, D.C., Bulletin 17B, variously paged.

Watson, K.M., and Schopp, R.D., 2009, Methodology for estimation of flood magnitude and frequency for New Jersey streams: U.S. Geological Survey Scientific Investigations Report 2009-5167, $51 \mathrm{p}$.

Wiley, J.B., and Atkins, J.T., Jr., 2010, Estimation of floodfrequency discharges for rural, unregulated streams in West Virginia: U.S. Geological Survey Scientific Investigations Report 2010-5033, 78 p.

Wiley, J.B., 2006, Low-flow analysis and selected flow statistics representative of 1930-2002 for streamflow-gaging stations in or near West Virginia: U.S. Geological Survey Scientific Investigations Report 2006-5002, 190 p.

Wolock, D.M., 2003, Flow characteristics at U.S. Geological Survey streamgages in the conterminous United States: U.S. Geological Survey Open-File Report 03-146, digital data set, available at http://water.usgs.gov/lookup/ getspatial?qsitesdd. 
Tables 1-6 
Table 1. List of U.S. Geological Survey streamgage locations in and near Pennsylvania with updated streamflow statistics.

[Latitude and Longitude in decimal degrees; $\mathrm{mi}^{2}$, square miles]

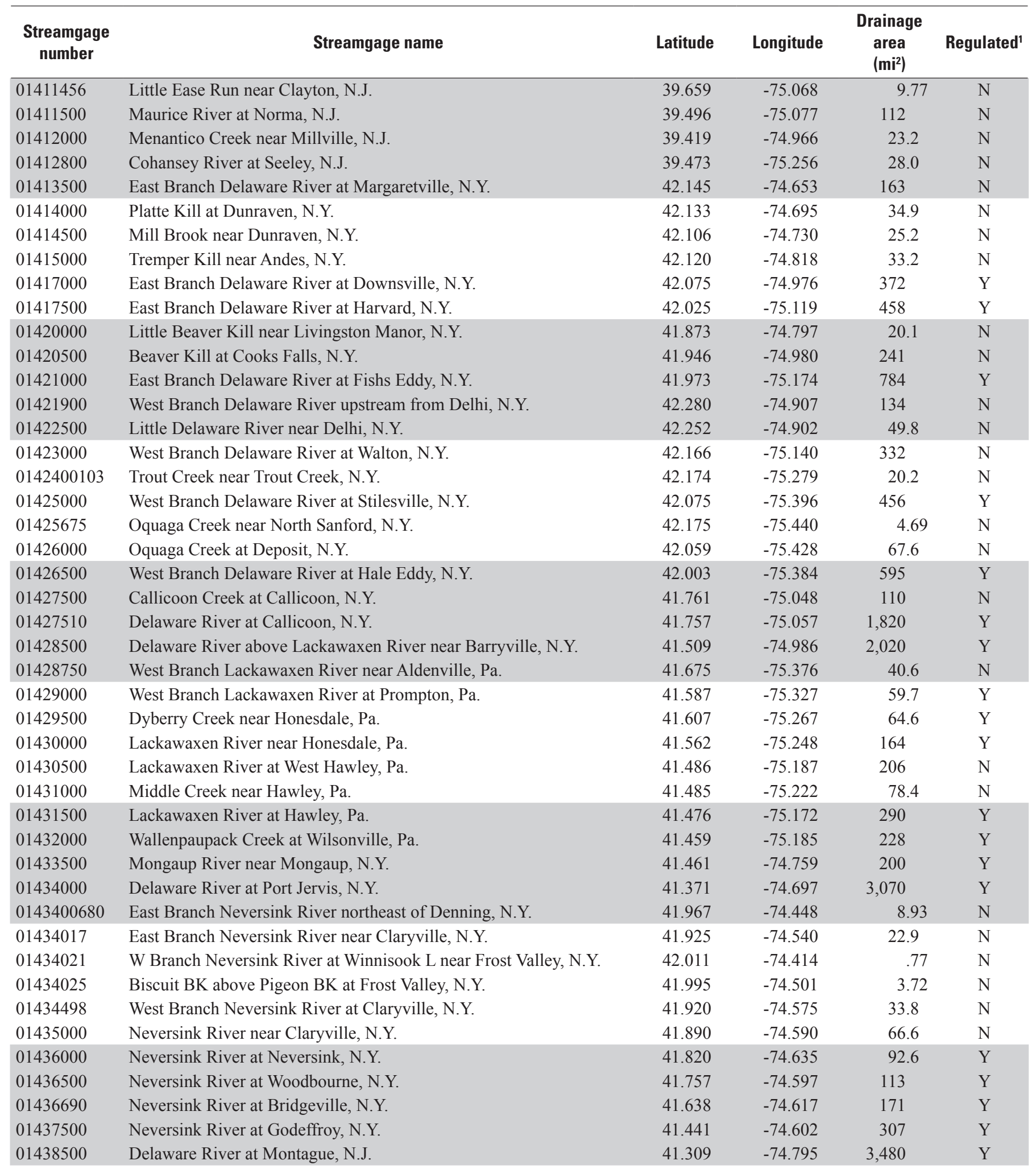


Table 1. List of U.S. Geological Survey streamgage locations in and near Pennsylvania with updated streamflow statistics.-Continued [Latitude and Longitude in decimal degrees; $\mathrm{mi}^{2}$, square miles]

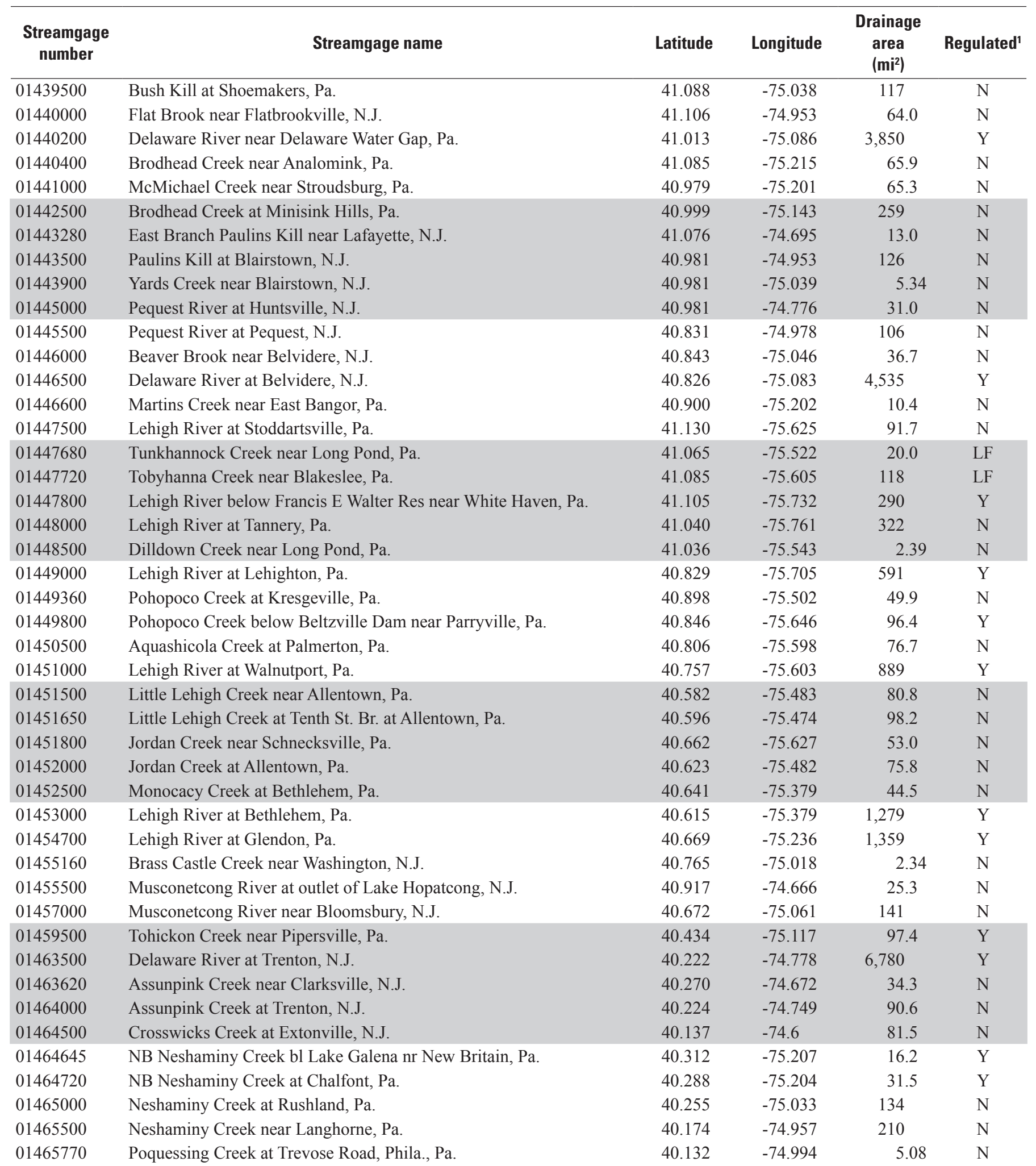


Table 1. List of U.S. Geological Survey streamgage locations in and near Pennsylvania with updated streamflow statistics.-Continued [Latitude and Longitude in decimal degrees; $\mathrm{mi}^{2}$, square miles]

\begin{tabular}{|c|c|c|c|c|c|}
\hline $\begin{array}{c}\text { Streamgage } \\
\text { number }\end{array}$ & Streamgage name & Latitude & Longitude & $\begin{array}{c}\text { Drainage } \\
\text { area } \\
\left(\mathrm{mi}^{2}\right)\end{array}$ & Regulated $^{1}$ \\
\hline 01465780 & Poquessing Creek above Byberry Creek at Phila., Pa. & 40.070 & -74.975 & 13.2 & $\mathrm{~N}$ \\
\hline 01465798 & Poquessing Creek at Grant Ave. at Philadelphia, Pa. & 40.057 & -74.985 & 21.4 & $\mathrm{~N}$ \\
\hline 01465850 & South Branch Rancocas Creek at Vincentown, N.J. & 39.94 & -74.763 & 64.5 & $\mathrm{~N}$ \\
\hline 01466500 & McDonalds Branch in Byrne State Forest, N.J. & 39.885 & -74.505 & 2.35 & $\mathrm{~N}$ \\
\hline 01467048 & Pennypack Creek at Lower Rhawn St Bdg, Phila., Pa. & 40.050 & -75.033 & 49.8 & $\mathrm{~N}$ \\
\hline 01467050 & Wooden Bridge Run at Philadelphia, Pa. & 40.055 & -75.022 & 3.35 & $\mathrm{~N}$ \\
\hline 01467081 & South Branch Pennsauken Creek at Cherry Hill, N.J. & 39.942 & -75.001 & 8.98 & $\mathrm{~N}$ \\
\hline 01467086 & Tacony Creek ab Adams Avenue, Philadelphia, Pa. & 40.047 & -75.111 & 16.7 & $\mathrm{~N}$ \\
\hline 01467087 & Frankford Creek at Castor Ave, Philadelphia, Pa. & 40.016 & -75.097 & 30.4 & $\mathrm{~N}$ \\
\hline 01468500 & Schuylkill River at Landingville, Pa. & 40.629 & -76.125 & 133 & $\mathrm{~N}$ \\
\hline 01469500 & Little Schuylkill River at Tamaqua, $\mathrm{Pa}$. & 40.807 & -75.972 & 42.9 & $\mathrm{~N}$ \\
\hline 01470500 & Schuylkill River at Berne, Pa. & 40.523 & -75.998 & 355 & $\mathrm{~N}$ \\
\hline 01470756 & Maiden Creek at Virginville, Pa. & 40.514 & -75.883 & 159 & $\mathrm{~N}$ \\
\hline 01470779 & Tulpehocken Creek near Bernville, Pa. & 40.413 & -76.172 & 66.5 & $\mathrm{~N}$ \\
\hline 01470853 & Furnace Creek at Robesonia, Pa. & 40.340 & -76.143 & 4.18 & $\mathrm{~N}$ \\
\hline 01470960 & Tulpehocken Creek at Blue Marsh Damsite near Reading, Pa. & 40.371 & -76.025 & 175 & Y \\
\hline 01471000 & Tulpehocken Creek near Reading, Pa. & 40.369 & -75.979 & 211 & $\mathrm{Y}$ \\
\hline 01471510 & Schuylkill River at Reading, $\mathrm{Pa}$. & 40.335 & -75.936 & 880 & $\mathrm{Y}$ \\
\hline 01471875 & Manatawny Creek near Spangsville, $\mathrm{Pa}$. & 40.340 & -75.742 & 56.9 & $\mathrm{~N}$ \\
\hline 01472620 & East Branch Perkiomen Creek near Dublin, Pa. & 40.404 & -75.234 & 4.05 & LF \\
\hline 01472810 & East Branch Perkiomen Creek near Schwenksville, Pa. & 40.259 & -75.429 & 58.7 & LF \\
\hline 01473000 & Perkiomen Creek at Graterford, $\mathrm{Pa}$. & 40.230 & -75.452 & 279 & LF \\
\hline 01473120 & Skippack Creek near Collegeville, $\mathrm{Pa}$. & 40.165 & -75.433 & 53.7 & $\mathrm{~N}$ \\
\hline 01473169 & Valley Creek at Pa. Turnpike Br near Valley Forge, $\mathrm{Pa}$. & 40.079 & -75.461 & 20.8 & $\mathrm{~N}$ \\
\hline 01473500 & Schuylkill River at Norristown, Pa. & 40.111 & -75.347 & 1,760 & $\mathrm{~N}$ \\
\hline 01473900 & Wissahickon Creek at Fort Washington, Pa. & 40.124 & -75.220 & 40.8 & $\mathrm{~N}$ \\
\hline 01473950 & Wissahickon Creek at Bells Mill Rd, Phila., Pa. & 40.080 & -75.226 & 53.6 & $\mathrm{~N}$ \\
\hline 01473980 & Wissahickon Creek at Livezey Lane, Phila., Pa. & 40.050 & -75.214 & 59.2 & $\mathrm{~N}$ \\
\hline 01474000 & Wissahickon Creek at Mouth, Philadelphia, Pa. & 40.015 & -75.207 & 64.0 & $\mathrm{~N}$ \\
\hline 01474500 & Schuylkill River at Philadelphia, Pa. & 39.968 & -75.189 & 1,893 & $\mathrm{~N}$ \\
\hline 01475000 & Mantua Creek at Pitman, N.J. & 39.737 & -75.113 & 6.05 & $\mathrm{~N}$ \\
\hline 01475300 & Darby Creek at Waterloo Mills near Devon, Pa. & 40.023 & -75.422 & 5.15 & $\mathrm{~N}$ \\
\hline 01475510 & Darby Creek near Darby, Pa. & 39.929 & -75.272 & 37.4 & $\mathrm{~N}$ \\
\hline
\end{tabular}


Table 1. List of U.S. Geological Survey streamgage locations in and near Pennsylvania with updated streamflow statistics. - Continued [Latitude and Longitude in decimal degrees; $\mathrm{mi}^{2}$, square miles]

\begin{tabular}{|c|c|c|c|c|c|}
\hline $\begin{array}{c}\text { Streamgage } \\
\text { number }\end{array}$ & Streamgage name & Latitude & Longitude & $\begin{array}{c}\text { Drainage } \\
\text { area } \\
\left(\mathrm{mi}^{2}\right)\end{array}$ & Regulated $^{1}$ \\
\hline 01475530 & Cobbs Creek at U.S. Highway No. 1 at Philadelphia, Pa. & 39.975 & -75.280 & 4.78 & $\mathrm{~N}$ \\
\hline 01475550 & Cobbs Creek at Darby, Pa. & 39.917 & -75.247 & 22.0 & $\mathrm{~N}$ \\
\hline 01475850 & Crum Creek near Newtown Square, Pa. & 39.976 & -75.437 & 15.8 & $\mathrm{~N}$ \\
\hline 01477000 & Chester Creek near Chester, Pa. & 39.869 & -75.408 & 61.1 & $\mathrm{~N}$ \\
\hline 01477120 & Raccoon Creek near Swedesboro, N.J. & 39.741 & -75.259 & 26.9 & $\mathrm{~N}$ \\
\hline 01477800 & Shellpot Creek at Wilmington, Del. & 39.761 & -75.519 & 7.46 & $\mathrm{~N}$ \\
\hline 01478000 & Christina River at Coochs Bridge, Del. & 39.637 & -75.728 & 20.5 & $\mathrm{LF}$ \\
\hline 01478500 & White Clay Creek above Newark, Del. & 39.714 & -75.759 & 66.7 & $\mathrm{~N}$ \\
\hline 01480015 & Red Clay Creek near Stanton, Del. & 39.716 & -75.640 & 52.4 & $\mathrm{~N}$ \\
\hline 01480100 & Little Mill Creek at Elsmere, Del. & 39.735 & -75.587 & 6.70 & $\mathrm{~N}$ \\
\hline 01480300 & West Branch Brandywine Creek near Honey Brook, Pa. & 40.073 & -75.861 & 18.7 & $\mathrm{~N}$ \\
\hline 01480500 & West Branch Brandywine Creek at Coatesville, Pa. & 39.986 & -75.827 & 45.8 & $\mathrm{LF}$ \\
\hline 01480617 & West Branch Brandywine Creek at Modena, Pa. & 39.962 & -75.801 & 55.0 & $\mathrm{~N}$ \\
\hline 01480675 & Marsh Creek near Glenmoore, Pa. & 40.098 & -75.742 & 8.57 & $\mathrm{~N}$ \\
\hline 01480680 & Marsh Creek near Lyndell, Pa. & 40.066 & -75.727 & 17.8 & $\mathrm{~N}$ \\
\hline 01480685 & Marsh Creek near Downingtown, Pa. & 40.055 & -75.716 & 20.3 & $\mathrm{Y}$ \\
\hline 01480700 & East Branch Brandywine Creek near Downingtown, Pa. & 40.035 & -75.709 & 60.6 & $\mathrm{Y}$ \\
\hline 01480800 & East Branch Brandywine Creek at Downingtown, Pa. & 40.006 & -75.705 & 81.6 & $\mathrm{~N}$ \\
\hline 01484100 & Beaverdam Branch at Houston, Del. & 38.906 & -75.513 & 3.02 & $\mathrm{~N}$ \\
\hline 01484270 & Beaverdam Creek near Milton, Del. & 38.762 & -75.267 & 6.85 & $\mathrm{~N}$ \\
\hline 01493000 & Unicorn Branch near Millington, Md. & 39.250 & -75.861 & 19.7 & $\mathrm{~N}$ \\
\hline 01493500 & Morgan Creek near Kennedyville, Md. & 39.280 & -76.015 & 12.7 & $\mathrm{~N}$ \\
\hline 01495000 & Big Elk Creek at Elk Mills, Md. & 39.657 & -75.822 & 52.6 & $\mathrm{~N}$ \\
\hline 01496000 & NorthEast Creek at Leslie, Md. & 39.628 & -75.944 & 24.3 & $\mathrm{~N}$ \\
\hline 01496200 & Principio Creek near Principio Furnace, Md. & 39.626 & -76.041 & 9.03 & $\mathrm{~N}$ \\
\hline 01496500 & Oaks Creek at Index, N.Y. & 42.666 & -74.960 & 102 & $\mathrm{~N}$ \\
\hline 01500000 & Ouleout Creek at East Sidney, N.Y. & 42.333 & -75.235 & 103 & $\mathrm{Y}$ \\
\hline 01500500 & Susquehanna River at Unadilla, N.Y. & 42.321 & -75.317 & 982 & $\mathrm{~N}$ \\
\hline 01502000 & Butternut Creek at Morris, N.Y. & 42.545 & -75.239 & 59.7 & $\mathrm{~N}$ \\
\hline 01502500 & Unadilla River at Rockdale, N.Y. & 42.378 & -75.406 & 520 & $\mathrm{~N}$ \\
\hline 01503000 & Susquehanna River at Conklin, N.Y. & 42.035 & -75.803 & 2,232 & $\mathrm{~N}$ \\
\hline 01505000 & Chenango River at Sherburne, N.Y. & 42.679 & -75.510 & 263 & $\mathrm{~N}$ \\
\hline
\end{tabular}


Table 1. List of U.S. Geological Survey streamgage locations in and near Pennsylvania with updated streamflow statistics. - Continued [Latitude and Longitude in decimal degrees; $\mathrm{mi}^{2}$, square miles]

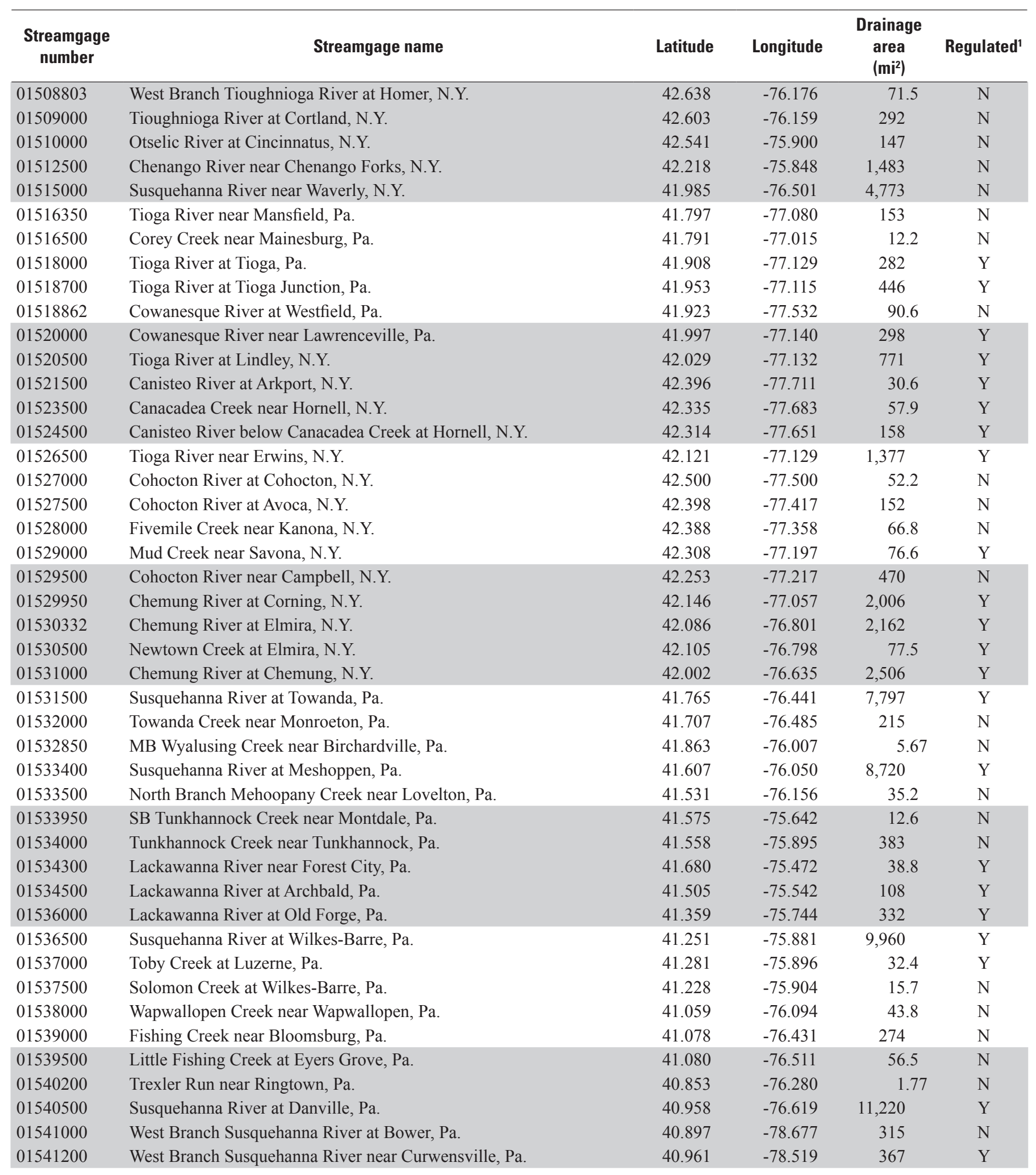


Table 1. List of U.S. Geological Survey streamgage locations in and near Pennsylvania with updated streamflow statistics. - Continued [Latitude and Longitude in decimal degrees; $\mathrm{mi}^{2}$, square miles]

\begin{tabular}{|c|c|c|c|c|c|}
\hline $\begin{array}{c}\text { Streamgage } \\
\text { number }\end{array}$ & Streamgage name & Latitude & Longitude & $\begin{array}{c}\text { Drainage } \\
\text { area } \\
\left(\mathrm{mi}^{2}\right)\end{array}$ & Regulated $^{1}$ \\
\hline 01541303 & West Branch Susquehanna River at Hyde, Pa. & 41.005 & -78.457 & 474 & $\mathrm{Y}$ \\
\hline 01541500 & Clearfield Creek at Dimeling, $\mathrm{Pa}$. & 40.972 & -78.406 & 371 & Y \\
\hline 01542000 & Moshannon Creek at Osceola Mills, Pa. & 40.850 & -78.268 & 68.8 & $\mathrm{~N}$ \\
\hline 01543000 & Driftwood Branch Sinnemahoning Creek at Sterling Run, Pa. & 41.413 & -78.197 & 272 & $\mathrm{~N}$ \\
\hline 01543500 & Sinnemahoning Creek at Sinnemahoning, Pa. & 41.317 & -78.103 & 685 & $\mathrm{~N}$ \\
\hline 01544000 & First Fork Sinnemahoning Creek near Sinnemahoning, $\mathrm{Pa}$. & 41.402 & -78.024 & 245 & $\mathrm{Y}$ \\
\hline 01544500 & Kettle Creek at Cross Fork, Pa. & 41.476 & -77.826 & 136 & $\mathrm{~N}$ \\
\hline 01545000 & Kettle Creek near Westport, Pa. & 41.320 & -77.874 & 233 & $\mathrm{Y}$ \\
\hline 01546400 & Spring Creek at Houserville, Pa. & 40.834 & -77.828 & 58.5 & $\mathrm{~N}$ \\
\hline 01546500 & Spring Creek near Axemann, Pa. & 40.890 & -77.794 & 87.2 & $\mathrm{~N}$ \\
\hline 01547100 & Spring Creek at Milesburg, Pa. & 40.932 & -77.786 & 142 & $\mathrm{~N}$ \\
\hline 01547200 & Bald Eagle Creek below Spring Creek at Milesburg, $\mathrm{Pa}$. & 40.943 & -77.786 & 265 & $\mathrm{~N}$ \\
\hline 01547500 & Bald Eagle Creek at Blanchard, Pa. & 41.052 & -77.604 & 339 & $\mathrm{Y}$ \\
\hline 01547700 & Marsh Creek at Blanchard, Pa. & 41.060 & -77.606 & 44.1 & $\mathrm{~N}$ \\
\hline 01547800 & South Fork Beech Creek near Snow Shoe, Pa. & 41.024 & -77.904 & 12.2 & $\mathrm{~N}$ \\
\hline 01547950 & Beech Creek at Monument, Pa. & 41.112 & -77.702 & 152 & $\mathrm{~N}$ \\
\hline 01548005 & Bald Eagle Creek near Beech Creek Station, Pa. & 41.081 & -77.549 & 562 & $\mathrm{Y}$ \\
\hline 01548500 & Pine Creek at Cedar Run, $\mathrm{Pa}$. & 41.522 & -77.447 & 604 & $\mathrm{~N}$ \\
\hline 01553130 & Sand Spring Run near White Deer, Pa. & 41.059 & -77.077 & 4.93 & $\mathrm{~N}$ \\
\hline 01553500 & West Branch Susquehanna River at Lewisburg, $\mathrm{Pa}$. & 40.968 & -76.876 & 6,847 & $\mathrm{Y}$ \\
\hline 01553700 & Chillisquaque Creek at Washingtonville, $\mathrm{Pa}$. & 41.062 & -76.680 & 51.3 & $\mathrm{~N}$ \\
\hline 01554000 & Susquehanna River at Sunbury, Pa. & 40.835 & -76.827 & 18,300 & $\mathrm{Y}$ \\
\hline 01554500 & Shamokin Creek near Shamokin, Pa. & 40.810 & -76.584 & 54.2 & $\mathrm{~N}$ \\
\hline 01555000 & Penns Creek at Penns Creek, Pa. & 40.867 & -77.048 & 301 & $\mathrm{~N}$ \\
\hline 01555500 & East Mahantango Creek near Dalmatia, Pa. & 40.611 & -76.912 & 162 & $\mathrm{~N}$ \\
\hline 01556000 & Frankstown Branch Juniata River at Williamsburg, Pa. & 40.463 & -78.200 & 291 & $\mathrm{~N}$ \\
\hline 01557500 & Bald Eagle Creek at Tyrone, Pa. & 40.684 & -78.234 & 44.1 & $\mathrm{~N}$ \\
\hline 01558000 & Little Juniata River at Spruce Creek, Pa. & 40.613 & -78.141 & 220 & $\mathrm{~N}$ \\
\hline 01559000 & Juniata River at Huntingdon, Pa. & 40.485 & -78.019 & 816 & $\mathrm{LF}$ \\
\hline 01559500 & Standing Stone Creek near Huntingdon, Pa. & 40.524 & -77.971 & 128 & $\mathrm{~N}$ \\
\hline 01559700 & Sulphur Springs Creek near Manns Choice, $\mathrm{Pa}$. & 39.978 & -78.619 & 5.28 & $\mathrm{~N}$ \\
\hline 01560000 & Dunning Creek at Belden, $\mathrm{Pa}$. & 40.072 & -78.493 & 172 & $\mathrm{~N}$ \\
\hline
\end{tabular}


Table 1. List of U.S. Geological Survey streamgage locations in and near Pennsylvania with updated streamflow statistics.-Continued [Latitude and Longitude in decimal degrees; $\mathrm{mi}^{2}$, square miles]

\begin{tabular}{|c|c|c|c|c|c|}
\hline $\begin{array}{c}\text { Streamgage } \\
\text { number }\end{array}$ & Streamgage name & Latitude & Longitude & $\begin{array}{c}\text { Drainage } \\
\text { area } \\
\left(\mathrm{mi}^{2}\right)\end{array}$ & Regulated $^{1}$ \\
\hline 01561000 & Brush Creek at Gapsville, Pa. & 39.956 & -78.254 & 36.8 & $\mathrm{~N}$ \\
\hline 01562000 & Raystown Branch Juniata River at Saxton, Pa. & 40.216 & -78.265 & 756 & $\mathrm{~N}$ \\
\hline 01562500 & Great Trough Creek near Marklesburg, Pa. & 40.350 & -78.130 & 84.6 & $\mathrm{~N}$ \\
\hline 01563200 & Raystown Branch Juniata River below Rays Dam nr Huntingdon, Pa. & 40.429 & -77.991 & 960 & $\mathrm{Y}$ \\
\hline 01565000 & Kishacoquillas Creek at Reedsville, Pa. & 40.655 & -77.583 & 164 & $\mathrm{~N}$ \\
\hline 01565700 & Little Lost Creek at Oakland Mills, Pa. & 40.605 & -77.311 & 6.52 & $\mathrm{~N}$ \\
\hline 01566000 & Tuscarora Creek near Port Royal, Pa. & 40.515 & -77.419 & 214 & $\mathrm{~N}$ \\
\hline 01566500 & Cocolamus Creek near Millerstown, $\mathrm{Pa}$. & 40.566 & -77.118 & 57.2 & $\mathrm{~N}$ \\
\hline 01567000 & Juniata River at Newport, $\mathrm{Pa}$. & 40.478 & -77.129 & 3,354 & $\mathrm{Y}$ \\
\hline 01569000 & Stony Creek nr Dauphin, Pa. & 40.380 & -76.907 & 33.2 & $\mathrm{~N}$ \\
\hline 01569800 & Letort Spring Run near Carlisle, Pa. & 40.235 & -77.139 & 21.6 & $\mathrm{~N}$ \\
\hline 01570000 & Conodoguinet Creek near Hogestown, Pa. & 40.252 & -77.021 & 470 & $\mathrm{LF}$ \\
\hline 01570500 & Susquehanna River at Harrisburg, $\mathrm{Pa}$. & 40.255 & -76.886 & 24,100 & $\mathrm{Y}$ \\
\hline 01571000 & Paxton Creek near Penbrook, Pa. & 40.308 & -76.850 & 11.2 & $\mathrm{~N}$ \\
\hline 01571500 & Yellow Breeches Creek near Camp Hill, Pa. & 40.225 & -76.898 & 213 & $\mathrm{~N}$ \\
\hline 01572000 & Lower Little Swatara Creek at Pine Grove, Pa. & 40.538 & -76.377 & 34.3 & $\mathrm{~N}$ \\
\hline 01572025 & Swatara Creek near Pine Grove, Pa. & 40.533 & -76.402 & 116 & $\mathrm{~N}$ \\
\hline 01572190 & Swatara Creek near Inwood, $\mathrm{Pa}$. & 40.479 & -76.531 & 167 & $\mathrm{~N}$ \\
\hline 01573000 & Swatara Creek at Harper Tavern, Pa. & 40.403 & -76.577 & 337 & $\mathrm{~N}$ \\
\hline 01575500 & Codorus Creek near York, $\mathrm{Pa}$ & 39.946 & -76.755 & 222 & $\mathrm{Y}$ \\
\hline 01576000 & Susquehanna River at Marietta, Pa. & 40.055 & -76.531 & 25,990 & $\mathrm{Y}$ \\
\hline 01576085 & Little Conestoga Creek near Churchtown, Pa. & 40.145 & -75.989 & 5.82 & $\mathrm{~N}$ \\
\hline 01576500 & Conestoga River at Lancaster, $\mathrm{Pa}$. & 40.050 & -76.277 & 324 & $\mathrm{~N}$ \\
\hline 01576754 & Conestoga River at Conestoga, $\mathrm{Pa}$. & 39.946 & -76.368 & 470 & $\mathrm{~N}$ \\
\hline 01578310 & Susquehanna River at Conowingo, Md. & 39.658 & -76.174 & 27,100 & $\mathrm{Y}$ \\
\hline 01578400 & Bowery Run near Quarryville, Pa. & 39.895 & -76.114 & 5.98 & $\mathrm{~N}$ \\
\hline 01580000 & Deer Creek at Rocks, Md. & 39.630 & -76.403 & 94.4 & $\mathrm{~N}$ \\
\hline 01581500 & Bynum Run at Bel Air, Md. & 39.541 & -76.330 & 8.52 & $\mathrm{~N}$ \\
\hline 01581700 & Winters Run near Benson, Md. & 39.520 & -76.373 & 34.8 & $\mathrm{~N}$ \\
\hline 01582000 & Little Falls at Blue Mount, Md. & 39.604 & -76.620 & 52.9 & $\mathrm{~N}$ \\
\hline 01582500 & Gunpowder Falls at Glencoe, Md. & 39.550 & -76.636 & 160 & $\mathrm{Y}$ \\
\hline 01583000 & Slade Run near Glyndon, Md. & 39.495 & -76.795 & 2.09 & $\mathrm{~N}$ \\
\hline 01583100 & Piney Run at Dover, Md. & 39.521 & -76.767 & 12.3 & $\mathrm{~N}$ \\
\hline
\end{tabular}


Table 1. List of U.S. Geological Survey streamgage locations in and near Pennsylvania with updated streamflow statistics.—Continued [Latitude and Longitude in decimal degrees; $\mathrm{mi}^{2}$, square miles]

\begin{tabular}{|c|c|c|c|c|c|}
\hline $\begin{array}{c}\text { Streamgage } \\
\text { number }\end{array}$ & Streamgage name & Latitude & Longitude & $\begin{array}{c}\text { Drainage } \\
\text { area } \\
\left(\mathrm{mi}^{2}\right)\end{array}$ & Regulated ${ }^{1}$ \\
\hline 01583500 & Western Run at Western Run, Md. & 39.511 & -76.677 & 59.8 & $\mathrm{~N}$ \\
\hline 01584050 & Long Green Creek at Glen Arm, Md. & 39.455 & -76.479 & 9.40 & $\mathrm{~N}$ \\
\hline 01584500 & Little Gunpowder Falls at Laurel Brook, Md. & 39.505 & -76.432 & 36.1 & $\mathrm{~N}$ \\
\hline 01585200 & West Branch Herring Run at Idlewylde, Md. & 39.374 & -76.584 & 2.13 & $\mathrm{~N}$ \\
\hline 01585300 & Stemmers Run at Rossville, Md. & 39.341 & -76.488 & 4.46 & $\mathrm{~N}$ \\
\hline 01585400 & Brien Run at Stemmers Run, Md. & 39.334 & -76.473 & 1.97 & $\mathrm{~N}$ \\
\hline 01585500 & Cranberry Branch near Westminster, Md. & 39.593 & -76.968 & 3.29 & LF \\
\hline 01586000 & North Branch Patapsco River at Cedarhurst, Md. & 39.504 & -76.885 & 56.6 & LF \\
\hline 01589000 & Patapsco River at Hollofield, Md. & 39.310 & -76.792 & 285 & $\mathrm{Y}$ \\
\hline 01589100 & East Branch Herbert Run at Arbutus, Md. & 39.24 & -76.692 & 2.47 & $\mathrm{~N}$ \\
\hline 01589300 & Gwynns Falls at Villa Nova, Md. & 39.346 & -76.733 & 32.5 & $\mathrm{~N}$ \\
\hline 01589330 & Dead Run at Franklintown, Md. & 39.311 & -76.717 & 5.52 & $\mathrm{~N}$ \\
\hline 01589440 & Jones Falls at Sorrento, Md. & 39.392 & -76.661 & 25.2 & $\mathrm{~N}$ \\
\hline 01589500 & Sawmill Creek at Glen Burnie, Md. & 39.17 & -76.631 & 4.97 & LF \\
\hline 01594930 & Laurel Run at Dobbin Road near Wilson, Md. & 39.244 & -79.428 & 8.23 & $\mathrm{~N}$ \\
\hline 01594936 & North Fork Sand Run near Wilson, Md. & 39.260 & -79.410 & 1.91 & $\mathrm{~N}$ \\
\hline 01594950 & McMillan F near Fort Pendleton, Md. & 39.277 & -79.390 & 2.30 & $\mathrm{~N}$ \\
\hline 01595000 & North Branch Potomac River at Steyer, Md. & 39.302 & -79.307 & 73.1 & $\mathrm{~N}$ \\
\hline 01598500 & North Branch Potomac River at Luke, Md. & 39.479 & -79.064 & 406 & $\mathrm{Y}$ \\
\hline 01599000 & Georges Creek at Franklin, Md. & 39.494 & -79.045 & 72.4 & $\mathrm{~N}$ \\
\hline 01600000 & North Branch Potomac River at Pinto, Md. & 39.567 & -78.840 & 607 & $\mathrm{Y}$ \\
\hline 01601000 & Wills Creek below Hyndman, Pa. & 39.812 & -78.716 & 146 & $\mathrm{~N}$ \\
\hline 01601500 & Wills Creek near Cumberland, Md. & 39.670 & -78.788 & 247 & $\mathrm{~N}$ \\
\hline 01603000 & North Branch Potomac River near Cumberland, Md. & 39.622 & -78.773 & 877 & $\mathrm{Y}$ \\
\hline 01603500 & Evitts Creek near Centerville, $\mathrm{Pa}$. & 39.790 & -78.646 & 30.2 & $\mathrm{~N}$ \\
\hline 01604500 & Patterson Creek near Headsville, W.Va. & 39.443 & -78.822 & 221 & $\mathrm{~N}$ \\
\hline 01609000 & Town Creek near Oldtown, Md. & 39.553 & -78.555 & 148 & $\mathrm{~N}$ \\
\hline 01610000 & Potomac River at Paw Paw, W.Va. & 39.539 & -78.456 & 3,129 & $\mathrm{~N}$ \\
\hline 01610155 & Sideling Hill Creek near Bellegrove, Md. & 39.650 & -78.344 & 102 & $\mathrm{~N}$ \\
\hline 01611500 & Cacapon River near Great Cacapon, W.Va. & 39.582 & -78.310 & 675 & $\mathrm{~N}$ \\
\hline 01613000 & Potomac River at Hancock, Md. & 39.698 & -78.178 & 4,064 & $\mathrm{~N}$ \\
\hline 01613050 & Tonoloway Creek near Needmore, Pa. & 39.898 & -78.132 & 10.7 & $\mathrm{~N}$ \\
\hline
\end{tabular}


Table 1. List of U.S. Geological Survey streamgage locations in and near Pennsylvania with updated streamflow statistics.-Continued [Latitude and Longitude in decimal degrees; $\mathrm{mi}^{2}$, square miles]

\begin{tabular}{|c|c|c|c|c|c|}
\hline $\begin{array}{c}\text { Streamgage } \\
\text { number }\end{array}$ & Streamgage name & Latitude & Longitude & $\begin{array}{c}\text { Drainage } \\
\text { area } \\
\left(\mathrm{mi}^{2}\right)\end{array}$ & Regulated $^{1}$ \\
\hline 01614000 & Back Creek near Jones Springs, W.Va. & 39.512 & -78.037 & 235 & $\mathrm{~N}$ \\
\hline 01614090 & Conococheague Creek near Fayetteville, Pa. & 39.930 & -77.439 & 5.05 & $\mathrm{~N}$ \\
\hline 01614500 & Conococheague Creek at Fairview, Md. & 39.716 & -77.825 & 494 & $\mathrm{~N}$ \\
\hline 01616500 & Opequon Creek near Martinsburg, W.Va. & 39.424 & -77.939 & 273 & $\mathrm{~N}$ \\
\hline 01618000 & Potomac River at Shepherdstown, W.Va. & 39.435 & -77.801 & 5,939 & $\mathrm{~N}$ \\
\hline 01619000 & Antietam Creek near Waynesboro, Pa. & 39.716 & -77.607 & 93.5 & $\mathrm{~N}$ \\
\hline 01619500 & Antietam Creek near Sharpsburg, Md. & 39.450 & -77.730 & 281 & $\mathrm{LF}$ \\
\hline 01637500 & Catoctin Creek near Middletown, Md. & 39.427 & -77.556 & 66.9 & $\mathrm{~N}$ \\
\hline 01639000 & Monocacy River at Bridgeport, Md. & 39.679 & -77.235 & 173 & $\mathrm{~N}$ \\
\hline 01640965 & Hunting Creek near Foxville, Md. & 39.620 & -77.466 & 2.14 & $\mathrm{~N}$ \\
\hline 01640970 & Hunting Creek Tributary near Foxville, Md. & 39.628 & -77.462 & 4.01 & $\mathrm{~N}$ \\
\hline 01641000 & Hunting Creek at Jimtown, Md. & 39.594 & -77.397 & 18.4 & $\mathrm{LF}$ \\
\hline 01641500 & Fishing Creek near Lewistown, Md. & 39.527 & -77.467 & 7.29 & $\mathrm{~N}$ \\
\hline 01642500 & Linganore Creek near Frederick, Md. & 39.415 & -77.333 & 82.3 & LF \\
\hline 01643000 & Monocacy River at Jug Bridge near Frederick, Md. & 39.403 & -77.366 & 817 & $\mathrm{~N}$ \\
\hline 01643500 & Bennett Creek at Park Mills, Md. & 39.294 & -77.407 & 62.8 & $\mathrm{~N}$ \\
\hline 03007800 & Allegheny River at Port Allegany, Pa. & 41.819 & -78.293 & 248 & $\mathrm{~N}$ \\
\hline 03008000 & Newell Creek near Port Allegany, $\mathrm{Pa}$. & 41.895 & -78.349 & 7.79 & $\mathrm{~N}$ \\
\hline 03009680 & Potato Creek at Smethport, Pa. & 41.810 & -78.430 & 160 & $\mathrm{~N}$ \\
\hline 03015000 & Conewango Creek at Russell, $\mathrm{Pa}$. & 41.938 & -79.133 & 816 & $\mathrm{Y}$ \\
\hline 03015280 & Jackson Run near North Warren, Pa. & 41.903 & -79.238 & 12.8 & $\mathrm{~N}$ \\
\hline 03015500 & Brokenstraw Creek at Youngsville, $\mathrm{Pa}$. & 41.853 & -79.317 & 321 & $\mathrm{~N}$ \\
\hline 03016000 & Allegheny River at West Hickory, Pa. & 41.571 & -79.408 & 3,660 & $\mathrm{Y}$ \\
\hline 03017500 & Tionesta Creek at Lynch, Pa. & 41.602 & -79.050 & 233 & $\mathrm{~N}$ \\
\hline 03020000 & Tionesta Creek at Tionesta Creek Dam, Pa. & 41.478 & -79.444 & 479 & $\mathrm{Y}$ \\
\hline 03020500 & Oil Creek at Rouseville, Pa. & 41.482 & -79.695 & 283 & $\mathrm{~N}$ \\
\hline 03021350 & French Creek near Wattsburg, Pa. & 42.015 & -79.783 & 92.0 & $\mathrm{~N}$ \\
\hline 03021410 & West Branch French Creek near Lowville, Pa. & 42.082 & -79.850 & 52.3 & $\mathrm{~N}$ \\
\hline 03021500 & French Creek at Carters Corners, Pa. & 41.956 & -79.877 & 208 & $\mathrm{~N}$ \\
\hline 03021520 & French Creek near Union City, Pa. & 41.908 & -79.897 & 221 & $\mathrm{Y}$ \\
\hline 03022540 & Woodcock Creek at Blooming Valley, Pa. & 41.691 & -80.048 & 31.1 & $\mathrm{~N}$ \\
\hline 03022554 & Woodcock Creek at Woodcock Creek Dam, Pa. & 41.696 & -80.108 & 45.6 & $\mathrm{Y}$ \\
\hline 03023100 & French Creek at Meadville, Pa. & 41.633 & -80.160 & 788 & Y \\
\hline
\end{tabular}


Table 1. List of U.S. Geological Survey streamgage locations in and near Pennsylvania with updated streamflow statistics. - Continued [Latitude and Longitude in decimal degrees; $\mathrm{mi}^{2}$, square miles]

\begin{tabular}{|c|c|c|c|c|c|}
\hline $\begin{array}{c}\text { Streamgage } \\
\text { number }\end{array}$ & Streamgage name & Latitude & Longitude & $\begin{array}{c}\text { Drainage } \\
\text { area } \\
\left(\mathrm{mi}^{2}\right)\end{array}$ & Regulated $^{1}$ \\
\hline 03024000 & French Creek at Utica, Pa. & 41.438 & -79.956 & 1,028 & $\mathrm{Y}$ \\
\hline 03025500 & Allegheny River at Franklin, Pa. & 41.390 & -79.820 & 5,982 & $\mathrm{Y}$ \\
\hline 03026500 & Sevenmile Run near Rasselas, $\mathrm{Pa}$. & 41.631 & -78.577 & 7.84 & $\mathrm{~N}$ \\
\hline 03028500 & Clarion River at Johnsonburg, Pa. & 41.486 & -78.678 & 204 & $\mathrm{Y}$ \\
\hline 03029500 & Clarion River at Cooksburg, $\mathrm{Pa}$. & 41.331 & -79.209 & 807 & LF \\
\hline 03030500 & Clarion River near Piney, $\mathrm{Pa}$. & 41.193 & -79.440 & 951 & $\mathrm{Y}$ \\
\hline 03031500 & Allegheny River at Parker, $\mathrm{Pa}$. & 41.101 & -79.681 & 7,671 & $\mathrm{Y}$ \\
\hline 03031950 & Big Run nr Sprankle Mills, Pa. & 40.992 & -79.090 & 7.38 & $\mathrm{~N}$ \\
\hline 03036000 & Mahoning Creek at Mahoning Creek Dam, Pa. & 40.928 & -79.291 & 344 & $\mathrm{Y}$ \\
\hline 03036500 & Allegheny River at Kittanning, $\mathrm{Pa}$. & 40.820 & -79.531 & 8,973 & Y \\
\hline 03038000 & Crooked Creek at Idaho, Pa. & 40.655 & -79.349 & 191 & LF \\
\hline 03039000 & Crooked Creek at Crooked Creek Dam near Ford City, Pa. & 40.720 & -79.511 & 278 & $\mathrm{Y}$ \\
\hline 03039200 & Clear Run near Buckstown, Pa. & 40.047 & -78.833 & 3.68 & $\mathrm{~N}$ \\
\hline 03039925 & North Fork Bens Creek at North Fork Reservoir, Pa. & 40.266 & -79.017 & 3.45 & $\mathrm{~N}$ \\
\hline 03040000 & Stonycreek River at Ferndale, $\mathrm{Pa}$. & 40.286 & -78.921 & 451 & $\mathrm{~N}$ \\
\hline 03041000 & Little Conemaugh River at East Conemaugh, Pa. & 40.346 & -78.883 & 183 & $\mathrm{~N}$ \\
\hline 03041500 & Conemaugh River at Seward, $\mathrm{Pa}$. & 40.419 & -79.026 & 715 & $\mathrm{~N}$ \\
\hline 03042000 & Blacklick Creek at Josephine, Pa. & 40.473 & -79.183 & 192 & $\mathrm{~N}$ \\
\hline 03047000 & Loyalhanna Creek at Loyalhanna Dam, Pa. & 40.459 & -79.449 & 290 & $\mathrm{Y}$ \\
\hline 03047500 & Kiskiminetas River at Avonmore, Pa. & 40.535 & -79.465 & 1,723 & $\mathrm{~N}$ \\
\hline 03048500 & Kiskiminetas River at Vandergrift, $\mathrm{Pa}$. & 40.605 & -79.552 & 1,825 & $\mathrm{Y}$ \\
\hline 03049000 & Buffalo Creek near Freeport, Pa. & 40.716 & -79.699 & 137 & $\mathrm{~N}$ \\
\hline 03049500 & Allegheny River at Natrona, Pa. & 40.615 & -79.718 & 11,410 & $\mathrm{Y}$ \\
\hline 03049800 & Little Pine Creek near Etna, Pa. & 40.520 & -79.938 & 5.78 & $\mathrm{~N}$ \\
\hline 03061500 & Buffalo Creek at Barrackville, W.Va. & 39.504 & -80.172 & 116 & $\mathrm{~N}$ \\
\hline 03062400 & Cobun Creek at Morgantown, W.Va. & 39.608 & -79.955 & 11.0 & $\mathrm{~N}$ \\
\hline 03062500 & Deckers Creek at Morgantown, W.Va. & 39.629 & -79.953 & 63.2 & $\mathrm{~N}$ \\
\hline 03065000 & Dry Fork at Hendricks, W.Va. & 39.072 & -79.623 & 349 & $\mathrm{~N}$ \\
\hline 03066000 & Blackwater River at Davis, W.Va. & 39.127 & -79.468 & 85.9 & $\mathrm{~N}$ \\
\hline 03068800 & Shavers Fork below Bowden, W.Va. & 38.913 & -79.770 & 151 & $\mathrm{~N}$ \\
\hline 03069000 & Shavers Fork at Parsons, W.Va. & 39.096 & -79.677 & 213 & $\mathrm{~N}$ \\
\hline 03069500 & Cheat River near Parsons, W.Va. & 39.123 & -79.681 & 722 & $\mathrm{~N}$ \\
\hline
\end{tabular}


Table 1. List of U.S. Geological Survey streamgage locations in and near Pennsylvania with updated streamflow statistics.-Continued [Latitude and Longitude in decimal degrees; $\mathrm{mi}^{2}$, square miles]

\begin{tabular}{|c|c|c|c|c|c|}
\hline $\begin{array}{c}\text { Streamgage } \\
\text { number }\end{array}$ & Streamgage name & Latitude & Longitude & $\begin{array}{c}\text { Drainage } \\
\text { area } \\
\left(\mathrm{mi}^{2}\right)\end{array}$ & Regulated $^{1}$ \\
\hline 03070000 & Cheat River at Rowlesburg, W.Va. & 39.346 & -79.665 & 939 & $\mathrm{~N}$ \\
\hline 03070500 & Big Sandy Creek at Rockville, W.Va. & 39.616 & -79.705 & 200 & $\mathrm{~N}$ \\
\hline 03072000 & Dunkard Creek at Shannopin, Pa. & 39.759 & -79.971 & 229 & $\mathrm{~N}$ \\
\hline 03073000 & South Fork Tenmile Creek at Jefferson, Pa. & 39.923 & -80.073 & 180 & $\mathrm{~N}$ \\
\hline 03074300 & Lick Run at Hopwood, Pa. & 39.868 & -79.694 & 3.80 & $\mathrm{~N}$ \\
\hline 03074500 & Redstone Creek at Waltersburg, $\mathrm{Pa}$. & 39.980 & -79.764 & 73.7 & $\mathrm{~N}$ \\
\hline 03075070 & Monongahela River at Elizabeth, Pa. & 40.262 & -79.901 & 5,340 & $\mathrm{Y}$ \\
\hline 03075500 & Youghiogheny River near Oakland, Md. & 39.422 & -79.424 & 134 & $\mathrm{~N}$ \\
\hline 03078000 & Casselman River at Grantsville, Md. & 39.702 & -79.136 & 62.5 & $\mathrm{~N}$ \\
\hline 03078500 & Big Piney Run near Salisbury, Pa. & 39.726 & -79.048 & 24.5 & $\mathrm{~N}$ \\
\hline 03079000 & Casselman River at Markleton, $\mathrm{Pa}$. & 39.860 & -79.228 & 382 & $\mathrm{~N}$ \\
\hline 03080000 & Laurel Hill Creek at Ursina, Pa. & 39.820 & -79.321 & 121 & $\mathrm{~N}$ \\
\hline 03081000 & Youghiogheny River below Confluence, $\mathrm{Pa}$. & 39.828 & -79.373 & 1,029 & $\mathrm{Y}$ \\
\hline 03082200 & Poplar Run near Normalville, $\mathrm{Pa}$. & 40.016 & -79.426 & 9.27 & $\mathrm{~N}$ \\
\hline 03082500 & Youghiogheny River at Connellsville, Pa. & 40.018 & -79.594 & 1,326 & Y \\
\hline 03083000 & Green Lick Run at Green Lick Reservoir, Pa. & 40.105 & -79.500 & 3.07 & $\mathrm{~N}$ \\
\hline 03083500 & Youghiogheny River at Sutersville, $\mathrm{Pa}$. & 40.240 & -79.806 & 1,715 & $\mathrm{Y}$ \\
\hline 03084000 & Abers Creek near Murrysville, $\mathrm{Pa}$. & 40.450 & -79.714 & 4.39 & $\mathrm{~N}$ \\
\hline 03092090 & West Branch Mahoning River near Ravenna, Ohio & 41.161 & -81.197 & 21.8 & $\mathrm{~N}$ \\
\hline 03092460 & West Branch Mahoning River at Wayland, Ohio & 41.157 & -81.072 & 81.7 & $\mathrm{Y}$ \\
\hline 03092500 & West Branch Mahoning River near Newton Falls, Ohio & 41.172 & -81.021 & 96.3 & Y \\
\hline 03093000 & Eagle Creek at Phalanx Station, Ohio & 41.261 & -80.954 & 97.6 & $\mathrm{~N}$ \\
\hline 03094000 & Mahoning River at Leavittsburg, Ohio & 41.239 & -80.881 & 575 & Y \\
\hline 03095500 & Mosquito Creek below Mosquito Creek Dam near Cortland, Ohio & 41.300 & -80.758 & 97.5 & $\mathrm{Y}$ \\
\hline 03097550 & Mahoning River at Ohio Edison P Plt at Niles, Ohio & 41.173 & -80.757 & 854 & $\mathrm{Y}$ \\
\hline 03098000 & Mahoning River at Youngstown, Ohio & 41.111 & -80.673 & 898 & $\mathrm{Y}$ \\
\hline 03098500 & Mill Creek at Youngstown, Ohio & 41.072 & -80.690 & 66.3 & $\mathrm{~N}$ \\
\hline 03098600 & Mahoning River below West Ave at Youngstown, Ohio & 41.105 & -80.663 & 978 & Y \\
\hline 03099500 & Mahoning River at Lowellville, Ohio & 41.037 & -80.536 & 1,073 & $\mathrm{Y}$ \\
\hline 03100000 & Shenango River near Turnersville, Pa. & 41.513 & -80.471 & 152 & $\mathrm{~N}$ \\
\hline 03101500 & Shenango River at Pymatuning Dam, Pa. & 41.498 & -80.460 & 167 & Y \\
\hline 03102000 & Shenango River near Jamestown, Pa. & 41.458 & -80.425 & 181 & $\mathrm{Y}$ \\
\hline
\end{tabular}


Table 1. List of U.S. Geological Survey streamgage locations in and near Pennsylvania with updated streamflow statistics. - Continued [Latitude and Longitude in decimal degrees; $\mathrm{mi}^{2}$, square miles]

\begin{tabular}{|c|c|c|c|c|c|}
\hline $\begin{array}{c}\text { Streamgage } \\
\text { number }\end{array}$ & Streamgage name & Latitude & Longitude & $\begin{array}{c}\text { Drainage } \\
\text { area } \\
\left(\mathrm{mi}^{2}\right)\end{array}$ & Regulated $^{1}$ \\
\hline 03102500 & Little Shenango River at Greenville, $\mathrm{Pa}$. & 41.422 & -80.376 & 104 & $\mathrm{~N}$ \\
\hline 03102950 & Pymatuning Creek at Kinsman, Ohio & 41.443 & -80.588 & 96.7 & $\mathrm{~N}$ \\
\hline 03103000 & Pymatuning Creek near Orangeville, $\mathrm{Pa}$. & 41.311 & -80.478 & 169 & $\mathrm{~N}$ \\
\hline 03104500 & Shenango River at New Castle, Pa. & 41.000 & -80.356 & 792 & $\mathrm{Y}$ \\
\hline 03104760 & Harthegig Run near Greenfield, Pa. & 41.186 & -80.327 & 2.26 & $\mathrm{~N}$ \\
\hline 03105500 & Beaver River at Wampum, Pa. & 40.889 & -80.337 & 2,235 & $\mathrm{Y}$ \\
\hline 03106000 & Connoquenessing Creek near Zelienople, $\mathrm{Pa}$. & 40.817 & -80.242 & 356 & $\mathrm{~N}$ \\
\hline 03106300 & Muddy Creek near Portersville, $\mathrm{Pa}$. & 40.963 & -80.125 & 51.2 & Y \\
\hline 03109500 & Little Beaver Creek near East Liverpool, Ohio & 40.676 & -80.541 & 496 & $\mathrm{~N}$ \\
\hline 03110000 & Yellow Creek near Hammondsville, Ohio & 40.538 & -80.725 & 147 & $\mathrm{~N}$ \\
\hline 03111150 & Brush Run near Buffalo, Pa. & 40.198 & -80.408 & 10.3 & $\mathrm{~N}$ \\
\hline 03111500 & Short Creek near Dillonvale, Ohio & 40.193 & -80.734 & 123 & $\mathrm{~N}$ \\
\hline 03111534 & Ohio River at Martins Ferry, Ohio & 40.105 & -80.708 & 24,620 & $\mathrm{~N}$ \\
\hline 03111548 & Wheeling Creek below Blaine, Ohio & 40.067 & -80.808 & 97.7 & $\mathrm{~N}$ \\
\hline 03112000 & Wheeling Creek at Elm Grove, W.Va. & 40.045 & -80.661 & 281 & $\mathrm{Y}$ \\
\hline 03114000 & Captina Creek at Armstrongs Mills, Ohio & 39.909 & -80.924 & 134 & $\mathrm{~N}$ \\
\hline 04209000 & Chagrin River at Willoughby, Ohio & 41.631 & -81.403 & 246 & $\mathrm{~N}$ \\
\hline 04212500 & Ashtabula River near Ashtabula, Ohio & 41.856 & -80.762 & 121 & $\mathrm{~N}$ \\
\hline
\end{tabular}

${ }^{1}$ Refers to regulation of streamflow during period of record of streamgage; N, no regulation during period of record; Y, regulation during period of record; $L F$, regulation for low flows during period of record. 
Table 2. Selected low-flow statistics for streamgage locations in and near Pennsylvania.

$\left[\mathrm{ft}^{3} / \mathrm{s}\right.$; cubic feet per second; - , statistic not computed; <, less than]

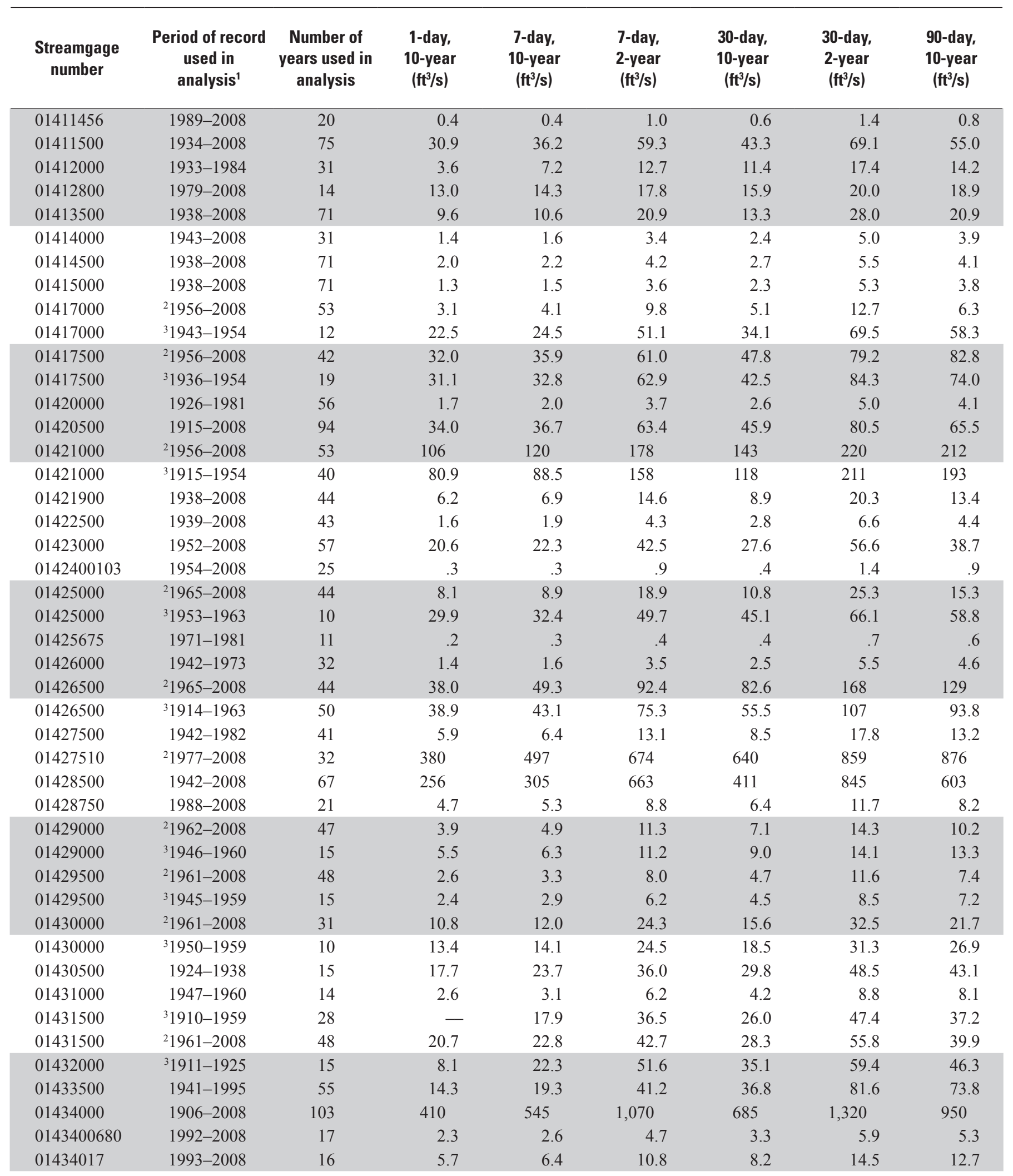


Table 2. Selected low-flow statistics for streamgage locations in and near Pennsylvania.-Continued

$\left[\mathrm{ft}^{3} / \mathrm{s}\right.$; cubic feet per second; - , statistic not computed; <, less than]

\begin{tabular}{|c|c|c|c|c|c|c|c|c|}
\hline 01434021 & $1992-2008$ & 17 & .1 & .1 & .2 & .1 & .3 & .3 \\
\hline 01434025 & 1985-2008 & 24 & .3 & .4 & 1.0 & .6 & 1.5 & 1.2 \\
\hline 01434498 & 1993-2008 & 16 & 7.0 & 7.4 & 15.2 & 9.4 & 19.5 & 15.2 \\
\hline 01436000 & ${ }^{3} 1943-1953$ & 11 & 18.3 & 19.9 & 33.6 & 25.2 & 43.4 & 42.2 \\
\hline 01436500 & ${ }^{2} 1955-1993$ & 34 & 11.6 & 13.7 & 20.4 & 16.9 & 26.4 & 24.3 \\
\hline 01436500 & ${ }^{3} 1939-1953$ & 15 & 20.4 & 22.0 & 37.4 & 29.1 & 49.2 & 44.4 \\
\hline 01436690 & 1994-2008 & 15 & 30.4 & 35.0 & 61.5 & 44.1 & 82.4 & 58.9 \\
\hline 01437500 & ${ }^{2} 1955-2008$ & 54 & 47.4 & 54.6 & 87.6 & 72.3 & 112 & 98.8 \\
\hline 01440000 & $1925-2008$ & 84 & 6.6 & 7.4 & 13.2 & 9.2 & 17.0 & 12.8 \\
\hline 01440200 & 1966-1995 & 30 & 1,030 & 1,200 & 1,830 & 1,440 & 2,110 & 1,660 \\
\hline 01440400 & 1959-2008 & 50 & 6.9 & 7.4 & 13.5 & 9.1 & 18.1 & 12.3 \\
\hline 01441000 & 1913-1938 & 26 & 13.3 & 16.2 & 24.3 & 19.1 & 29.8 & 23.3 \\
\hline 01442500 & $1952-2008$ & 57 & 44.7 & 48.4 & 80.6 & 57.0 & 100 & 73.6 \\
\hline 01443280 & 1994-2008 & 15 & 2.0 & 2.6 & 6.9 & 3.0 & 8.1 & 4.4 \\
\hline 01443500 & 1923-2008 & 84 & 11.6 & 16.2 & 28.3 & 20.3 & 36.8 & 26.8 \\
\hline 01443900 & 1968-2008 & 41 & .2 & .6 & 1.0 & .9 & 1.4 & 1.1 \\
\hline 01445000 & 1941-2008 & 27 & 1.9 & 2.1 & 5.3 & 2.8 & 7.2 & 4.8 \\
\hline 01445500 & 1923-2008 & 86 & 18.2 & 19.5 & 33.5 & 22.0 & 39.0 & 27.2 \\
\hline 01447800 & 1959-2008 & 50 & 39.5 & 46.8 & 81.4 & 62.4 & 124 & 94.1 \\
\hline 01448000 & 1918-1959 & 41 & 49.0 & 66.7 & 108 & 79.0 & 136 & 116 \\
\hline 01448500 & 1950-1996 & 47 & .4 & .4 & .8 & .5 & 1.0 & .7 \\
\hline 01449000 & 1984-2008 & 25 & 135 & 148 & 237 & 180 & 315 & 236 \\
\hline 01449360 & 1968-2008 & 41 & 14.4 & 15.1 & 23.7 & 17.3 & 27.7 & 21.7 \\
\hline 01449800 & 1969-2008 & 40 & 14.2 & 16.5 & 31.0 & 19.9 & 38.1 & 29.4 \\
\hline 01450500 & 1941-2008 & 68 & 15.1 & 17.0 & 29.3 & 20.0 & 35.6 & 26.7 \\
\hline 01451000 & ${ }^{2} 1962-2008$ & 47 & 185 & 203 & 337 & 252 & 430 & 322 \\
\hline 01451000 & ${ }^{3} 1948-1960$ & 13 & 203 & 213 & 311 & 253 & 391 & 343 \\
\hline 01451500 & 1947-2008 & 62 & 27.6 & 28.8 & 43.4 & 32.0 & 48.1 & 35.4 \\
\hline 01451650 & 1988-2008 & 21 & 29.8 & 36.2 & 52.7 & 41.9 & 60.7 & 50.0 \\
\hline 01451800 & $1967-2008$ & 42 & 1.7 & 2.2 & 7.0 & 3.7 & 11.0 & 7.5 \\
\hline 01452000 & 1946-2008 & 63 & 3.0 & 3.0 & 12.0 & 4.4 & 13.9 & 7.6 \\
\hline 01452500 & 1950-2008 & 59 & 12.1 & 13.2 & 22.9 & 14.8 & 25.9 & 16.8 \\
\hline 01453000 & ${ }^{2} 1929-2008$ & 81 & 340 & 371 & 579 & 439 & 702 & 546 \\
\hline
\end{tabular}


Table 2. Selected low-flow statistics for streamgage locations in and near Pennsylvania._Continued

$\left[\mathrm{ft}^{3} / \mathrm{s}\right.$; cubic feet per second; - , statistic not computed; <, less than]

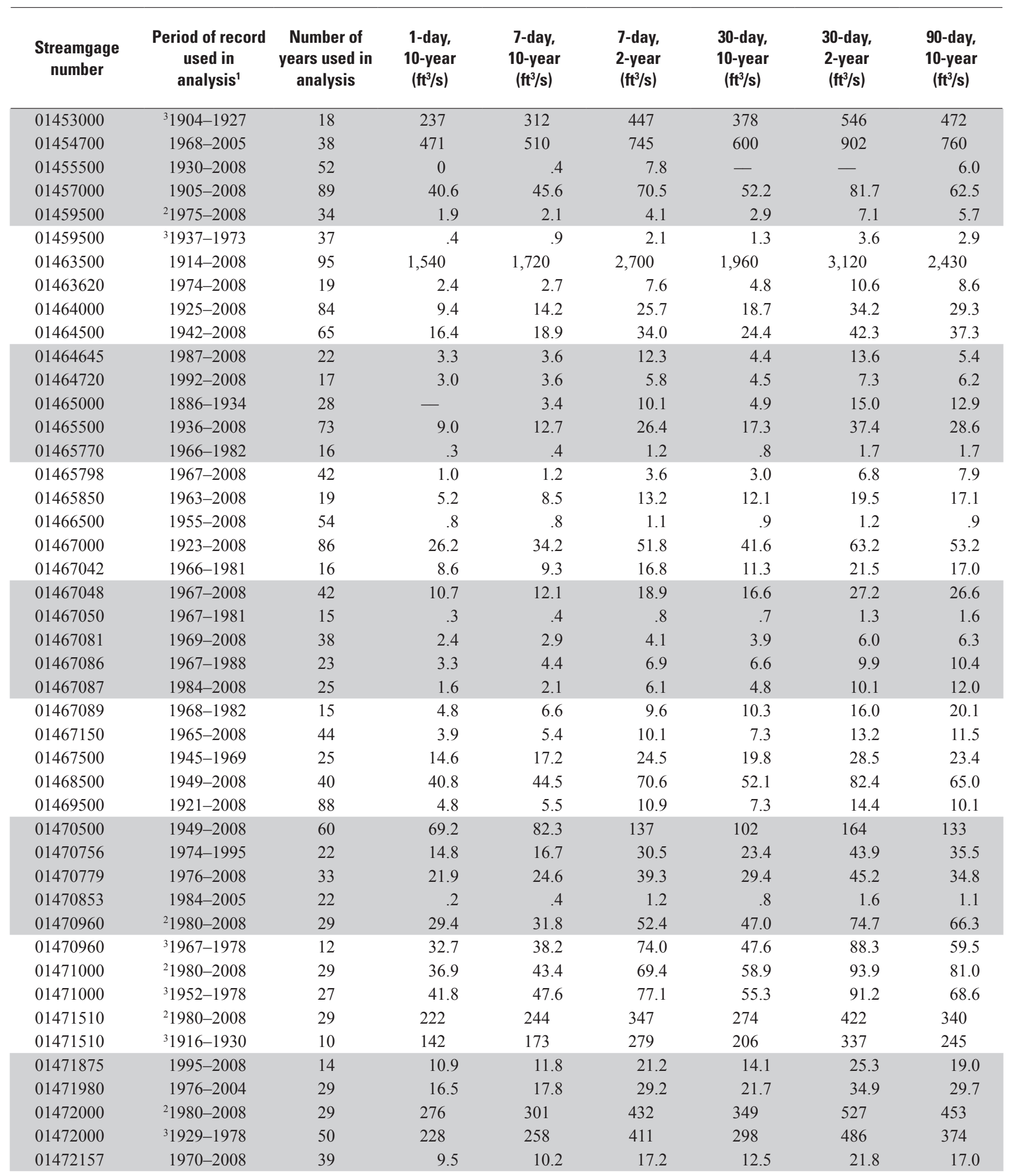


Table 2. Selected low-flow statistics for streamgage locations in and near Pennsylvania.-Continued

$\left[\mathrm{ft}^{3} / \mathrm{s}\right.$; cubic feet per second; - , statistic not computed; <, less than]

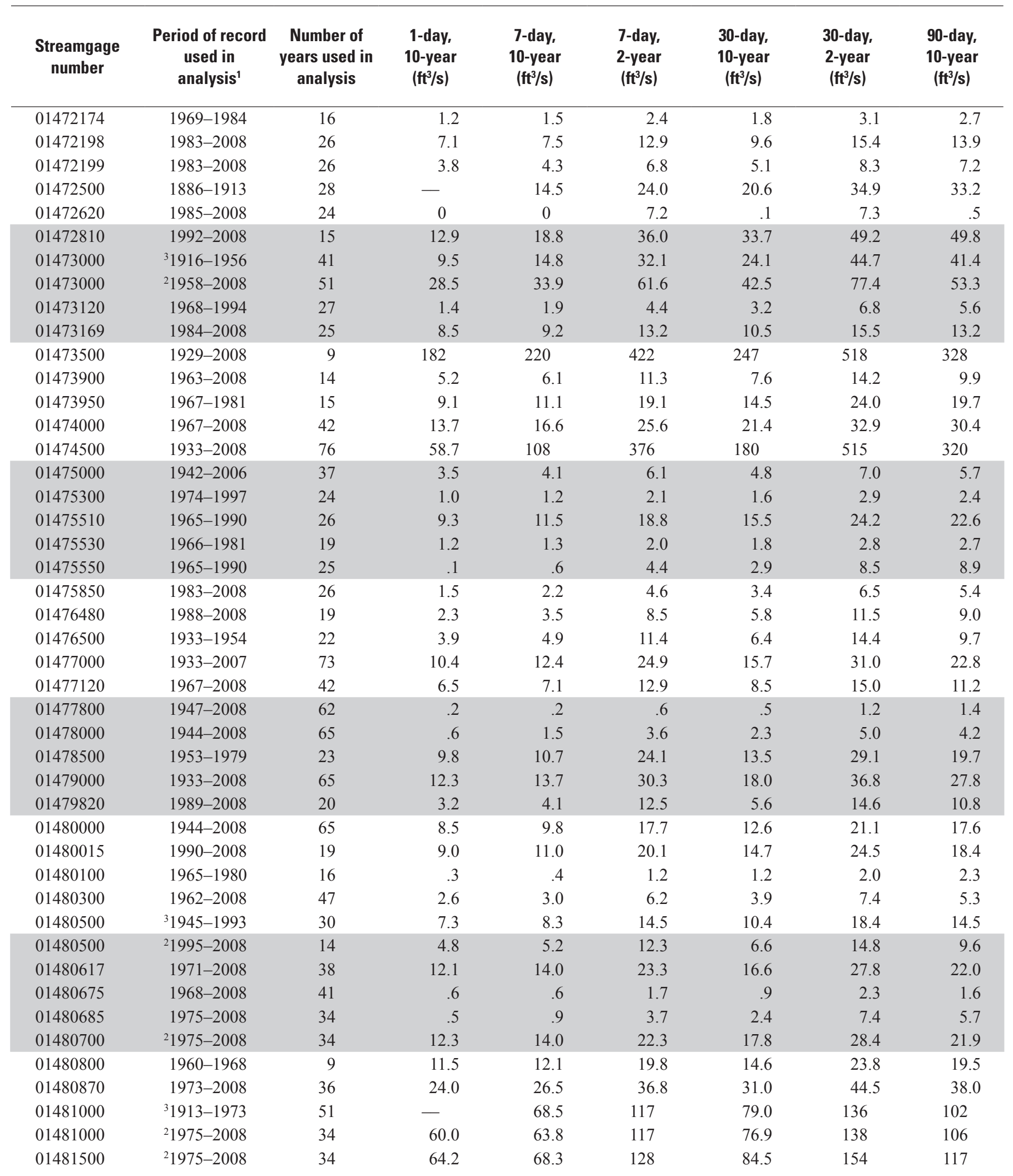


Table 2. Selected low-flow statistics for streamgage locations in and near Pennsylvania._Continued

$\left[\mathrm{ft}^{3} / \mathrm{s}\right.$; cubic feet per second; - , statistic not computed; <, less than]

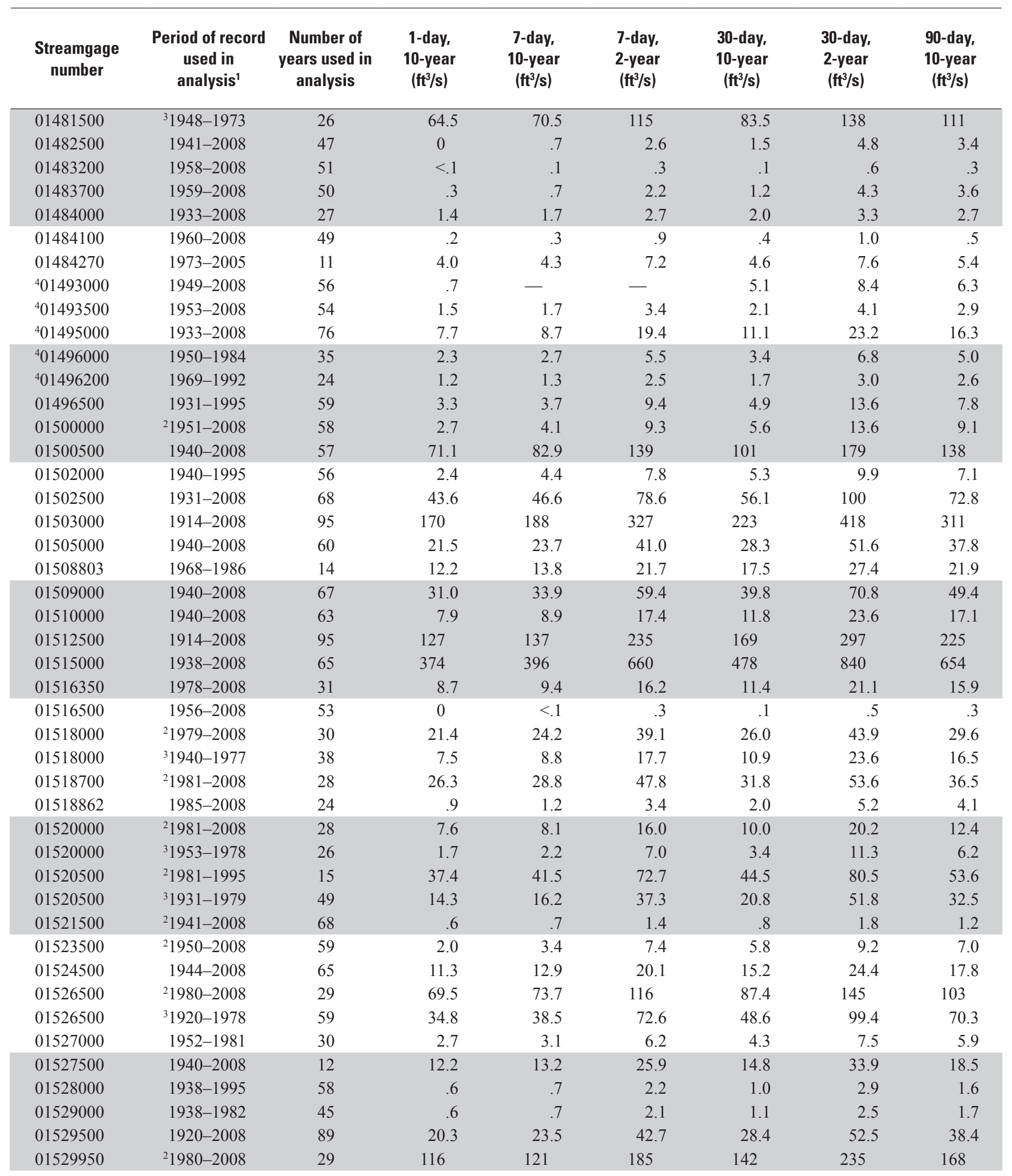


Table 2. Selected low-flow statistics for streamgage locations in and near Pennsylvania.-Continued

$\left[\mathrm{ft}^{3} / \mathrm{s}\right.$; cubic feet per second; - , statistic not computed; <, less than]

\begin{tabular}{|c|c|c|c|c|c|c|c|c|}
\hline 01530500 & 1940-2008 & 69 & 5.0 & 6.1 & 11.0 & 7.6 & 13 & 9.0 \\
\hline 01531000 & ${ }^{2} 1981-2008$ & 28 & 138 & 147 & 237 & 169 & 296 & 203 \\
\hline 01531000 & ${ }^{3} 1905-1979$ & 68 & 86.3 & 97.0 & 175 & 116 & 219 & 161 \\
\hline 01532000 & 1915-2008 & 94 & 2.2 & 2.8 & 9.7 & 4.6 & 14.4 & 9.4 \\
\hline 01532850 & 1967-1979 & 13 & .1 & .2 & .4 & .3 & .8 & .7 \\
\hline 01533400 & ${ }^{2} 1981-2008$ & 28 & 602 & 648 & 1,110 & 790 & 1,430 & 1,060 \\
\hline 01533500 & 1942-1958 & 17 & .4 & .6 & 1.5 & .8 & 2.0 & 1.7 \\
\hline 01533950 & 1962-1978 & 17 & .2 & .3 & 1.0 & .6 & 1.4 & 1.0 \\
\hline 01534500 & ${ }^{3} 1941-1959$ & 19 & 18.8 & 23.0 & 33.3 & 25.6 & 39.2 & 34.9 \\
\hline 01536000 & ${ }^{2} 1961-2008$ & 48 & 28.7 & 32.7 & 51.7 & 40.8 & 68.1 & 54.3 \\
\hline 01536000 & ${ }^{3} 1940-1959$ & 20 & 77.8 & 93.9 & 119 & 105 & 138 & 124 \\
\hline 01536500 & ${ }^{2} 1981-2008$ & 28 & 828 & 872 & 1,450 & 1,030 & 1,830 & 1,350 \\
\hline 01536500 & ${ }^{3} 1901-1979$ & 79 & 778 & 811 & 1,350 & 927 & 1,640 & 1,260 \\
\hline 01537000 & 1943-1993 & 51 & 1.3 & 2.0 & 4.9 & 3.1 & 6.4 & 4.7 \\
\hline 01537500 & 1941-1990 & 50 & .2 & .3 & 1.9 & .5 & 3.1 & 1.6 \\
\hline 01538000 & 1921-2008 & 88 & 3.1 & 3.6 & 7.1 & 5.0 & 9.3 & 7.5 \\
\hline 01539000 & 1940-2008 & 69 & 15.4 & 16.8 & 36.8 & 21.1 & 51.1 & 36.8 \\
\hline 01539500 & $1942-1958$ & 17 & .1 & .3 & 1.4 & 1.0 & 3.3 & 2.3 \\
\hline 01541308 & 1969-1979 & 11 & 1.3 & 1.3 & 1.9 & 1.6 & 2.4 & 2.1 \\
\hline 01541500 & ${ }^{2} 1962-2008$ & 47 & 39.0 & 41.9 & 66.5 & 51.9 & 86.3 & 70.6 \\
\hline 01541500 & ${ }^{3} 1915-1960$ & 46 & 14.9 & 21.3 & 41.9 & 28.5 & 55.0 & 42.9 \\
\hline 01542000 & 1942-1993 & 52 & 8.1 & 9.1 & 14.8 & 11.3 & 17.8 & 14.6 \\
\hline 01542500 & ${ }^{2} 1967-2008$ & 33 & 216 & 235 & 326 & 285 & 435 & 402 \\
\hline 01542500 & ${ }^{3} 1941-1965$ & 20 & - & 131 & 189 & 152 & 243 & 221 \\
\hline 01542810 & 1966-2008 & 43 & .1 & .1 & .3 & .2 & .5 & .3 \\
\hline 01543000 & 1915-2008 & 94 & 2.9 & 4.2 & 16.0 & 9.6 & 27.4 & 19.2 \\
\hline 01543500 & 1940-2008 & 69 & 10.7 & 14.5 & 44.9 & 26.6 & 74.9 & 50.5 \\
\hline 01544000 & ${ }^{2} 1957-2008$ & 52 & 3.3 & 6.9 & 19.0 & 11.2 & 31.1 & 19.0 \\
\hline 01544500 & 1942-2008 & 67 & 4.2 & 4.9 & 12.5 & 7.5 & 17.4 & 11.7 \\
\hline 01545000 & ${ }^{2} 1964-2008$ & 45 & 6.8 & 8.2 & 21.2 & 12.0 & 32.7 & 20.7 \\
\hline 01545500 & ${ }^{2} 1963-2008$ & 46 & 217 & 238 & 446 & 306 & 629 & 428 \\
\hline 01545500 & ${ }^{3} 1909-1961$ & 53 & 125 & 141 & 278 & 190 & 387 & 296 \\
\hline 01545600 & 1966-2008 & 43 & 1.2 & 1.5 & 4.4 & 2.4 & 6.7 & 4.2 \\
\hline
\end{tabular}


Table 2. Selected low-flow statistics for streamgage locations in and near Pennsylvania._Continued

$\left[\mathrm{ft}^{3} / \mathrm{s}\right.$; cubic feet per second; - , statistic not computed; <, less than]

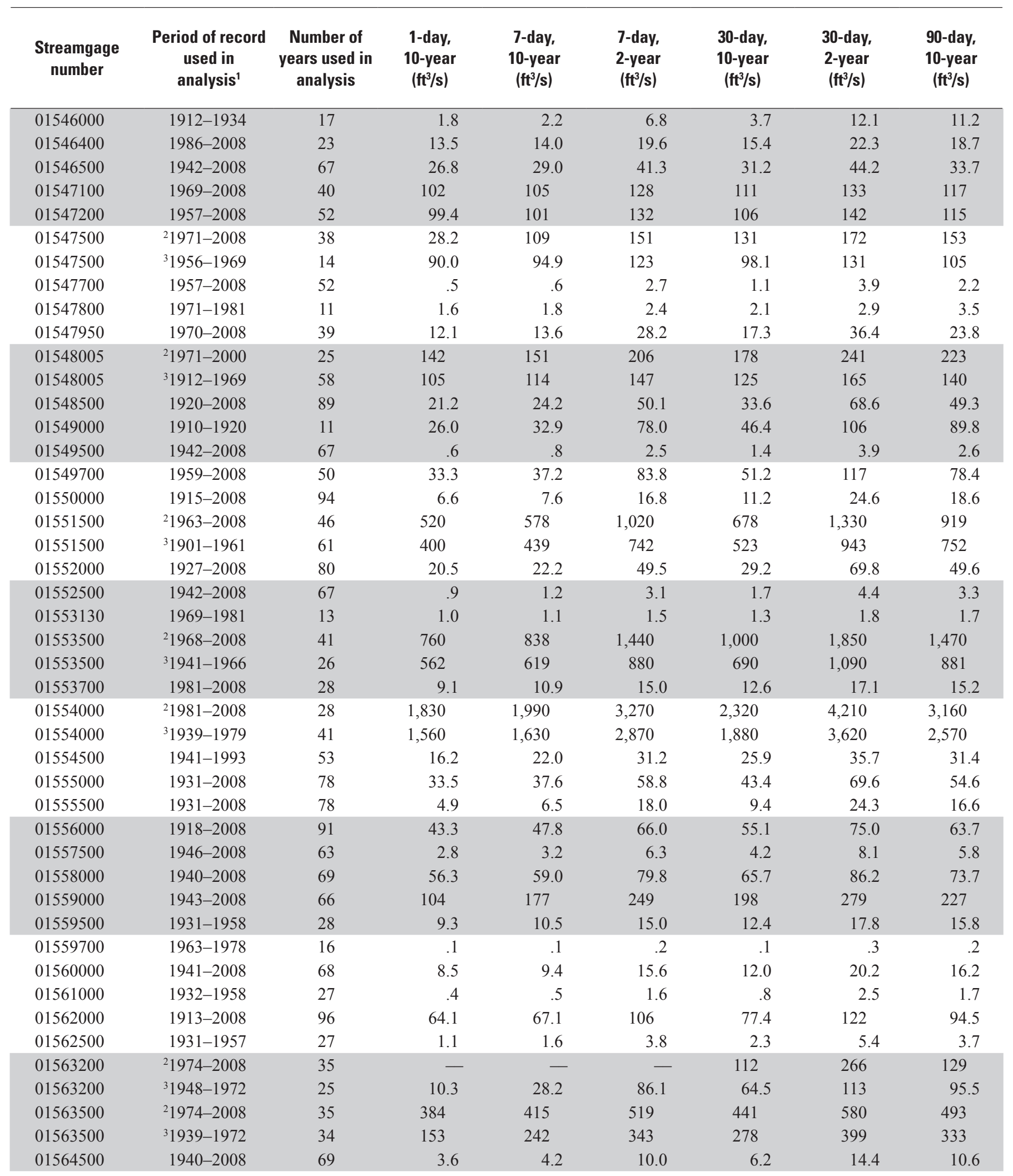


Table 2. Selected low-flow statistics for streamgage locations in and near Pennsylvania.-Continued

$\left[\mathrm{ft}^{3} / \mathrm{s}\right.$; cubic feet per second; - , statistic not computed; <, less than]

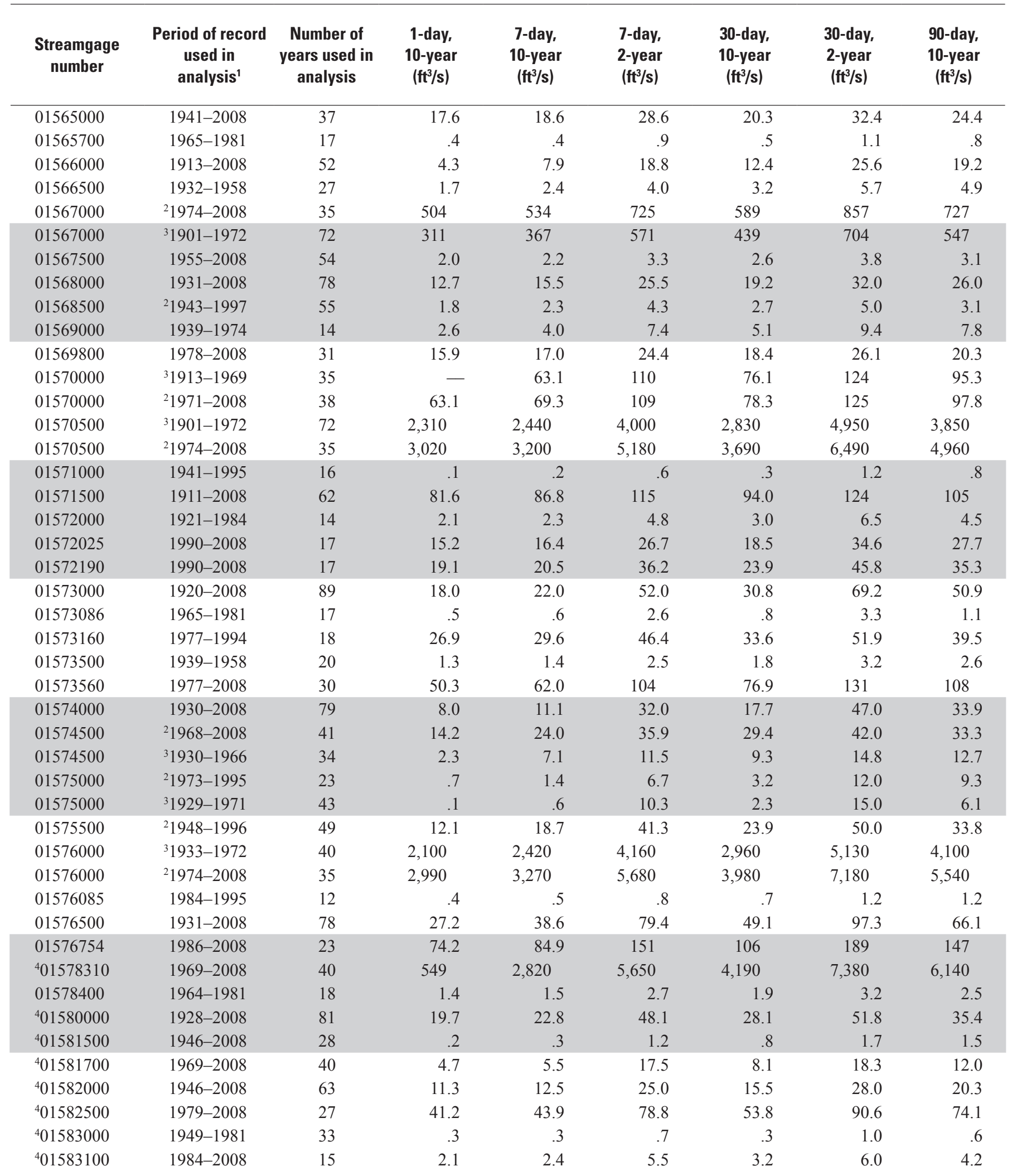


Table 2. Selected low-flow statistics for streamgage locations in and near Pennsylvania._Continued

$\left[\mathrm{ft}^{3} / \mathrm{s}\right.$; cubic feet per second; - , statistic not computed; <, less than]

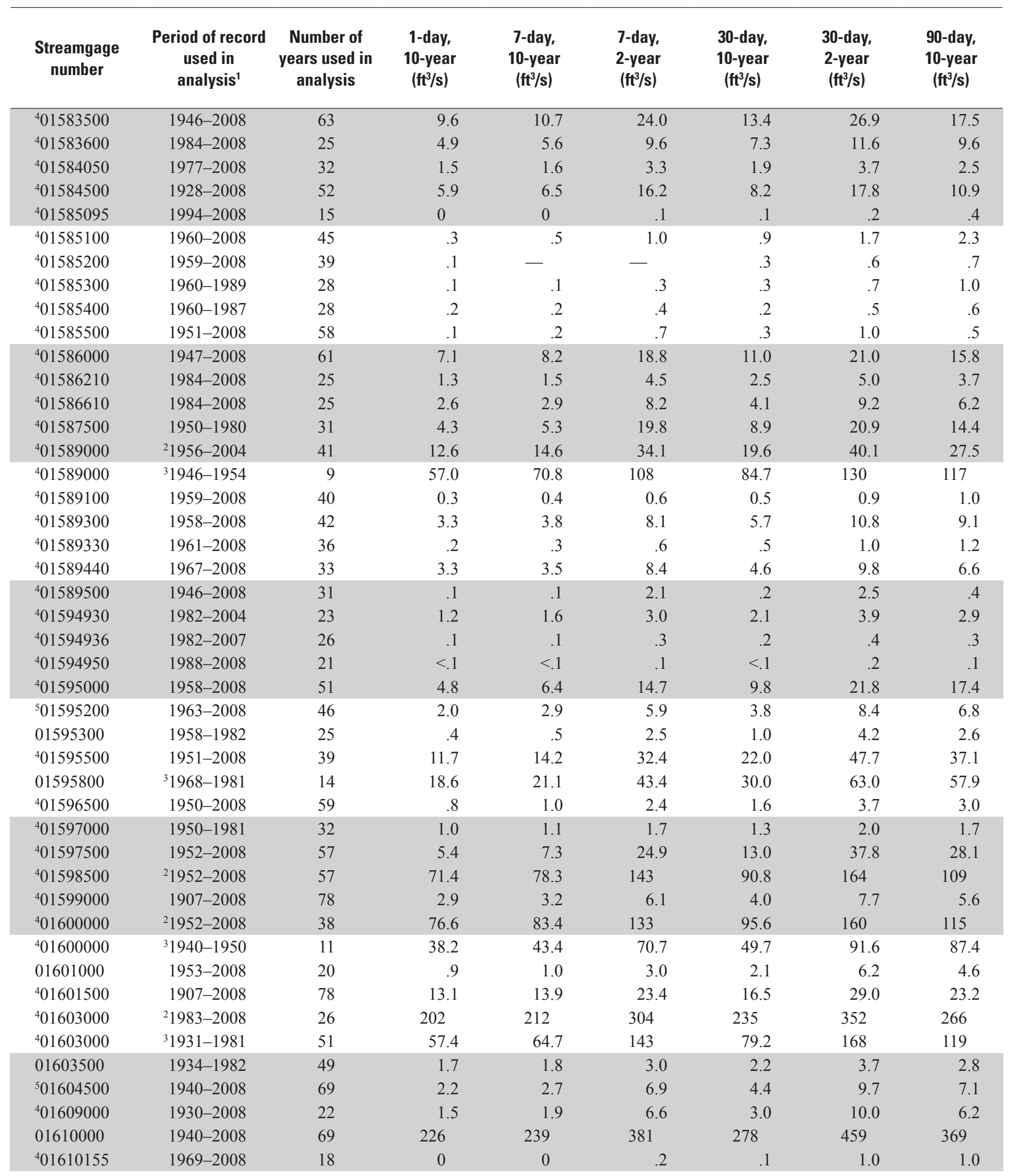


Table 2. Selected low-flow statistics for streamgage locations in and near Pennsylvania.-Continued

$\left[\mathrm{ft}^{3} / \mathrm{s}\right.$; cubic feet per second; - , statistic not computed; <, less than]

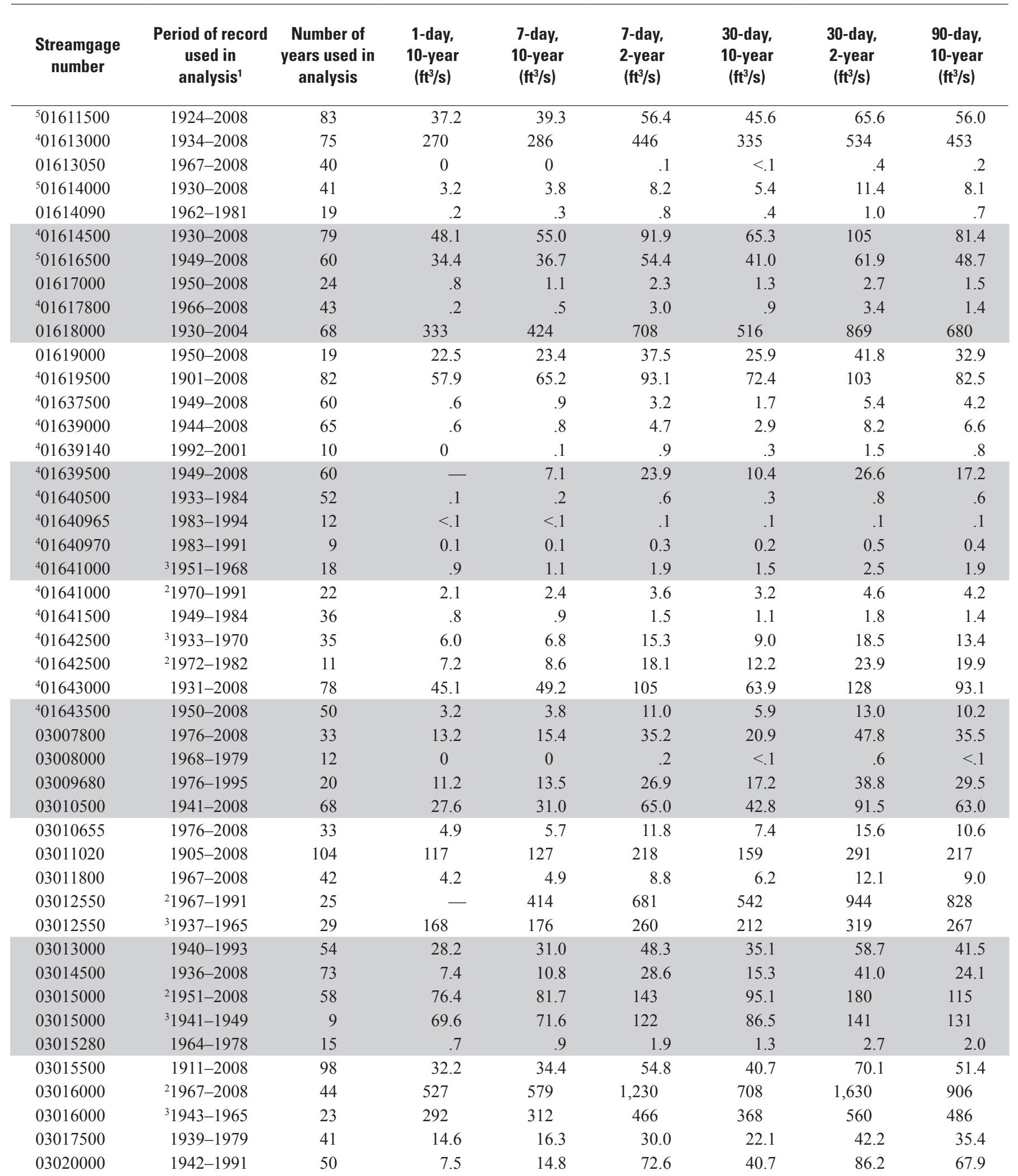


Table 2. Selected low-flow statistics for streamgage locations in and near Pennsylvania._Continued

$\left[\mathrm{ft}^{3} / \mathrm{s}\right.$; cubic feet per second; - , statistic not computed; <, less than]

\begin{tabular}{|c|c|c|c|c|c|c|c|c|}
\hline 03020500 & 1934-2008 & 75 & 27.4 & 30.1 & 50.2 & 37.4 & 66.0 & 48.4 \\
\hline 03021350 & 1976-2008 & 33 & 3.8 & 4.5 & 10.7 & 7.6 & 19.2 & 12.9 \\
\hline 03021410 & 1976-1993 & 18 & 2.3 & 2.8 & 5.6 & 4.6 & 10.6 & 8.6 \\
\hline 03021520 & 1973-1991 & 19 & 20.0 & 23.1 & 35.3 & 31.8 & 52.2 & 49.8 \\
\hline 03022540 & 1976-1995 & 16 & 2.5 & 2.8 & 4.9 & 3.8 & 7.1 & 5.6 \\
\hline 03022554 & 1976-1991 & 16 & 5.2 & 5.7 & 9.1 & 6.6 & 10.5 & 10.5 \\
\hline 03023100 & 1990-2008 & 19 & 50.4 & 55.3 & 110 & 67.6 & 161 & 104 \\
\hline 03024000 & ${ }^{2} 1972-2008$ & 37 & 77.4 & 85.4 & 166 & 109 & 233 & 176 \\
\hline 03026500 & 1953-2008 & 56 & .2 & .2 & .7 & .4 & 1.1 & .7 \\
\hline 03027500 & ${ }^{2} 1954-1991$ & 38 & - & 15.7 & 27.0 & 19.7 & 35.4 & 27.9 \\
\hline 03028000 & 1955-2008 & 54 & 6.0 & 6.6 & 12.3 & 8.4 & 16.6 & 12.0 \\
\hline 03028500 & ${ }^{2} 1954-1995$ & 42 & 55.5 & 65.2 & 109 & 79.2 & 139 & 112 \\
\hline 03029500 & ${ }^{3} 1940-1952$ & 13 & 51.9 & 57.0 & 96.4 & 69.9 & 123 & 103 \\
\hline 03029500 & ${ }^{2} 1954-2008$ & 55 & 151 & 171 & 282 & 195 & 328 & 244 \\
\hline 03030500 & 1949-2008 & 60 & 16.9 & 76.8 & 232 & 148 & 366 & 210 \\
\hline 03031500 & ${ }^{2} 1967-2008$ & 42 & 1,670 & 2,050 & 3,020 & 2,390 & 3,620 & 2,790 \\
\hline 03031500 & ${ }^{3} 1934-1965$ & 32 & 565 & 669 & 990 & 837 & 2,350 & 1,150 \\
\hline 03031950 & 1965-1981 & 17 & .3 & .4 & 1.0 & .7 & 1.4 & 1.1 \\
\hline 03038000 & ${ }^{3} 1939-1968$ & 30 & 3.5 & 5.5 & 9.6 & 7.6 & 14.4 & 11.6 \\
\hline 03039000 & ${ }^{2} 1941-1991$ & 51 & 4.7 & 6.6 & 17.6 & 12.0 & 28.9 & 23.1 \\
\hline 03039000 & ${ }^{3} 1920-1939$ & 40 & 1.6 & 3.0 & 11.5 & 6.2 & 21.8 & 14.3 \\
\hline 03039200 & 1966-1978 & 13 & 0 & .1 & .2 & .2 & .4 & .2 \\
\hline 03039925 & 1986-1998 & 10 & .2 & .3 & .8 & .4 & 1.2 & .6 \\
\hline 03040000 & 1915-2008 & 89 & 15.6 & 17.7 & 45.3 & 24.4 & 63.1 & 39.9 \\
\hline 03041000 & 1940-2006 & 56 & 6.8 & 8.6 & 27.7 & 11.7 & 37.8 & 21.1 \\
\hline 03041500 & 1940-2008 & 69 & 156 & 167 & 231 & 190 & 269 & 223 \\
\hline 03042000 & $1953-2008$ & 56 & 23.2 & 26.6 & 45.4 & 32.6 & 58.5 & 42.1 \\
\hline 03042200 & 1962-1988 & 18 & .2 & .3 & .6 & .4 & 1.0 & .6 \\
\hline 03042280 & ${ }^{2} 1973-2008$ & 36 & 5.1 & 6.0 & 9.8 & 7.5 & 12.2 & 9.9 \\
\hline 03042500 & ${ }^{2} 1970-2008$ & 39 & 34.2 & 39.0 & 50.7 & 45.6 & 62.9 & 55.4 \\
\hline 03042500 & ${ }^{3} 1953-1968$ & 16 & 9.2 & 11.3 & 15.0 & 13.3 & 22.0 & 16.6 \\
\hline 03043000 & 1909-1951 & 43 & 16.8 & 19.5 & 44.0 & 28.3 & 59.6 & 48.4 \\
\hline 03044000 & ${ }^{2} 1953-1991$ & 39 & - & - & - & 273 & 444 & 340 \\
\hline
\end{tabular}


Table 2. Selected low-flow statistics for streamgage locations in and near Pennsylvania.-Continued

[ $\mathrm{ft}^{3} / \mathrm{s}$; cubic feet per second; - , statistic not computed; <, less than]

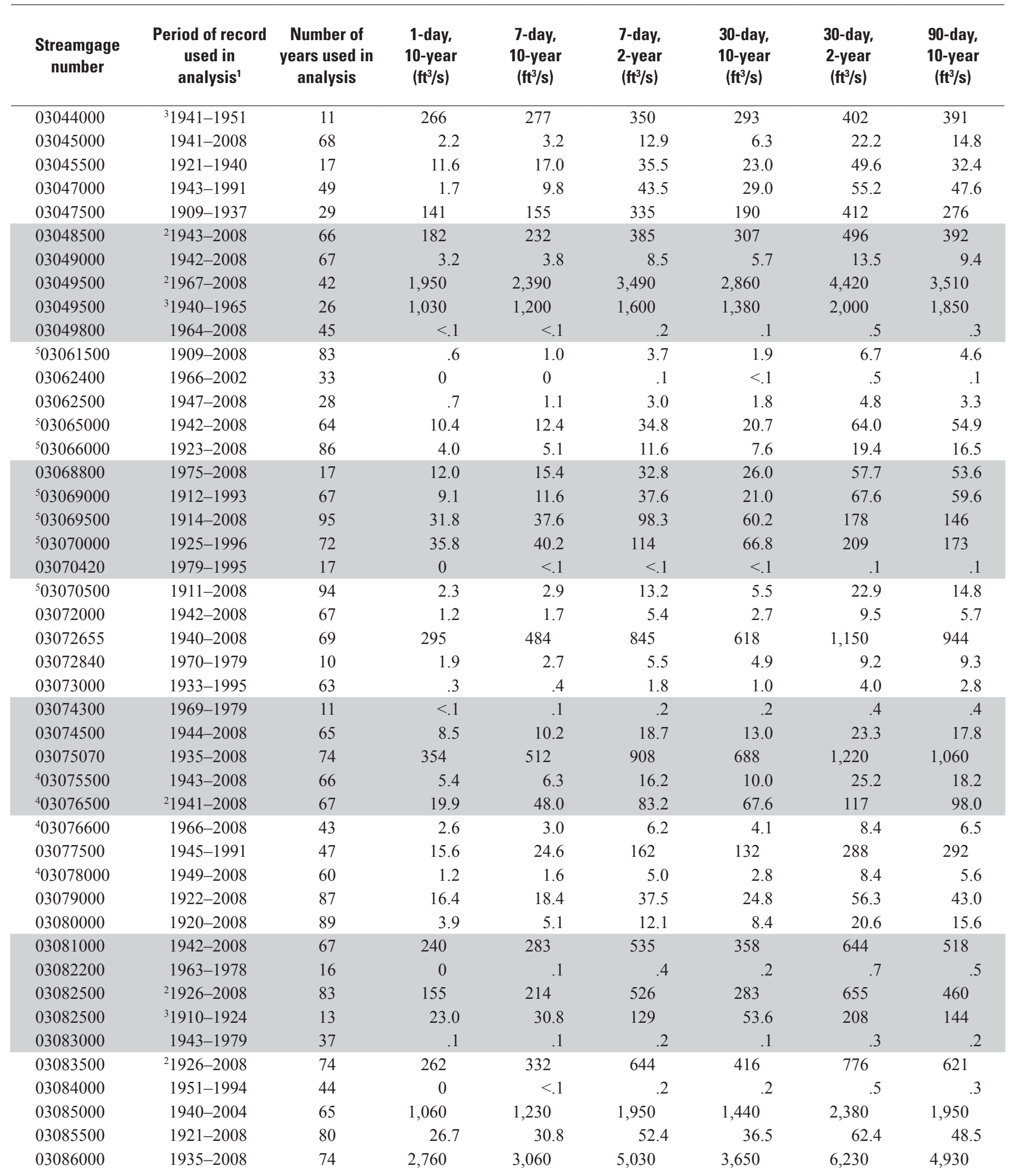


Table 2. Selected low-flow statistics for streamgage locations in and near Pennsylvania._-Continued

$\left[\mathrm{ft}^{3} / \mathrm{s}\right.$; cubic feet per second; - , statistic not computed; <, less than]

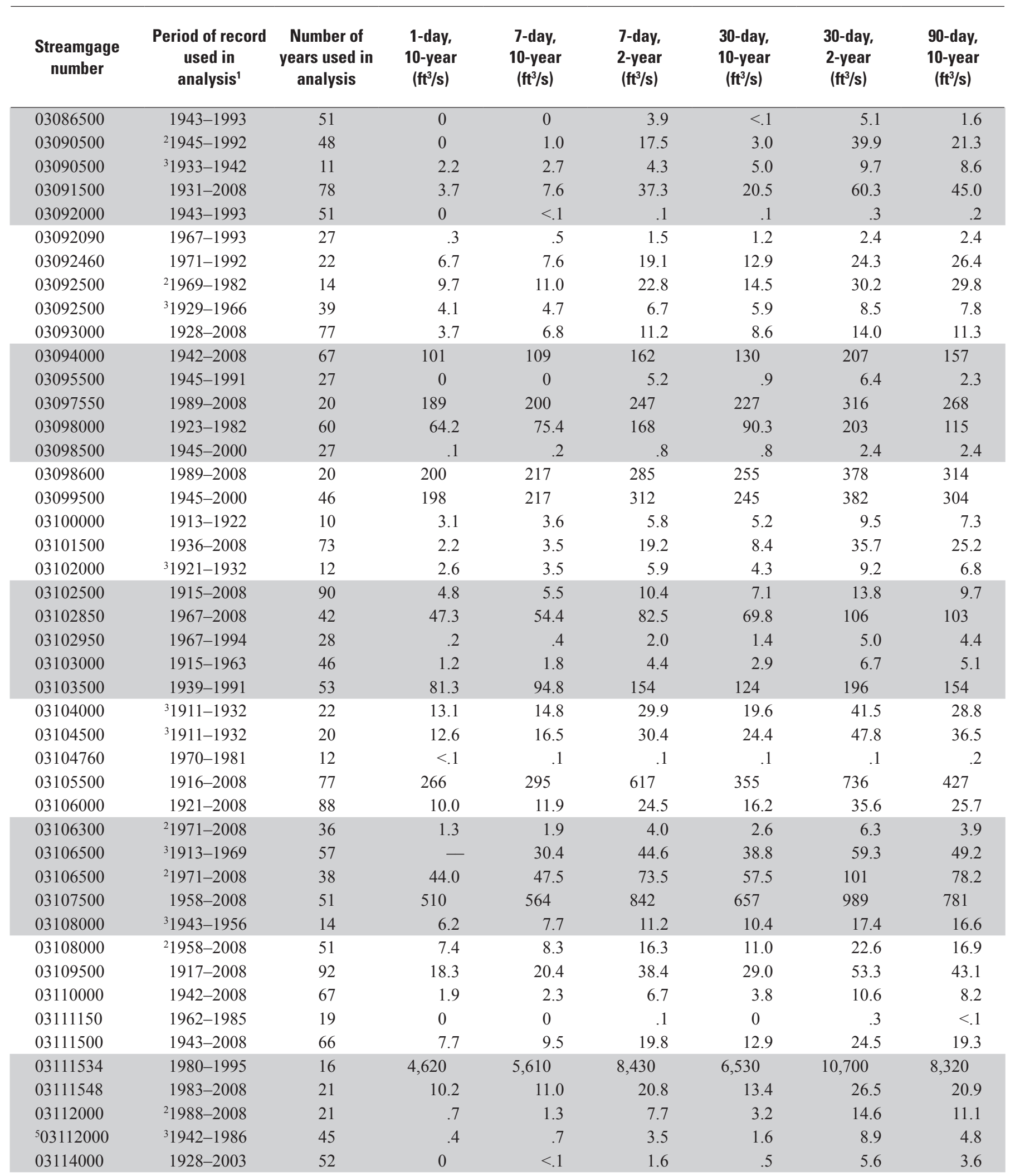


Table 2. Selected low-flow statistics for streamgage locations in and near Pennsylvania.-Continued

$\left[\mathrm{ft}^{3} / \mathrm{s}\right.$; cubic feet per second; - , statistic not computed; <, less than]

\begin{tabular}{|c|c|c|c|c|c|c|c|c|}
\hline $\begin{array}{c}\text { Streamgage } \\
\text { number }\end{array}$ & $\begin{array}{c}\text { Period of record } \\
\text { used in } \\
\text { analysis }\end{array}$ & $\begin{array}{l}\text { Number of } \\
\text { years used in } \\
\text { analysis }\end{array}$ & $\begin{array}{c}\text { 1-day, } \\
\text { 10-year } \\
\left(\mathrm{ft}^{3} / \mathrm{s}\right)\end{array}$ & $\begin{array}{l}\text { 7-day, } \\
\text { 10-year } \\
\left(\mathrm{ft}^{3} / \mathrm{s}\right)\end{array}$ & $\begin{array}{l}\text { 7-day, } \\
\text { 2-year } \\
\left(\mathrm{ft}^{3} / \mathrm{s}\right)\end{array}$ & $\begin{array}{c}\text { 30-day, } \\
\text { 10-year } \\
\left(\mathrm{ft}^{3} / \mathrm{s}\right)\end{array}$ & $\begin{array}{c}\text { 30-day, } \\
\text { 2-year } \\
\left(\mathrm{ft}^{3} / \mathrm{s}\right)\end{array}$ & $\begin{array}{c}\text { 90-day, } \\
10 \text {-year } \\
\left(\mathrm{ft}^{3} / \mathrm{s}\right)\end{array}$ \\
\hline 04209000 & 1927-2008 & 68 & 11.3 & 14.3 & 30.8 & 21.7 & 45.1 & 30.0 \\
\hline 04212500 & 1926-1979 & 47 & 0 & 0 & 0 & 0 & .4 & .2 \\
\hline 04213000 & 1924-2008 & 70 & 1.6 & 2.4 & 7.4 & 4.4 & 12.4 & 7.4 \\
\hline 04213075 & 1988-2008 & 21 & .3 & .3 & .7 & .5 & 1.0 & .8 \\
\hline 04221000 & $1957-2008$ & 37 & 15.6 & 17.2 & 30.7 & 21.6 & 41.0 & 29.8 \\
\hline 04223000 & 1910-2008 & 99 & 46.2 & 57.8 & 102 & 74.6 & 137 & 110 \\
\hline 04224775 & 1976-2008 & 33 & 7.2 & 7.6 & 11.5 & 8.4 & 14.6 & 10.4 \\
\hline 04227000 & $1960-2008$ & 44 & 19.6 & 21.8 & 37.0 & 25.7 & 45.6 & 30.3 \\
\hline
\end{tabular}

${ }^{1}$ Climatic year is defined as 12-month period beginning April 1 and ending March 31. The climatic year is designated by the calendar year in which it ends.

${ }^{2}$ Period of record for post-regulation period.

${ }^{3}$ Period of record for pre-regulation period.

${ }^{4}$ Low-flow statistics also presented in Doheny and Banks (2010).

${ }^{5}$ Low-flow statistics computed from a common period of record can be found in Wiley (2006). 
Table 3. Selected base-flow statistics for streamgage locations in and near Pennsylvania.

$\left[\mathrm{ft}^{3} / \mathrm{s}\right.$; cubic feet per second; - , statistic not computed]

\begin{tabular}{|c|c|c|c|c|c|}
\hline $\begin{array}{c}\text { Streamgage } \\
\text { number }\end{array}$ & $\begin{array}{l}\text { Period of } \\
\text { record used } \\
\text { in analysis }\end{array}$ & $\begin{array}{l}\text { Number of } \\
\text { years used } \\
\text { in analysis }\end{array}$ & $\begin{array}{c}10 \text {-year } \\
\text { base flow } \\
\left(\mathrm{ft}^{3} / \mathrm{s}\right)\end{array}$ & $\begin{array}{c}25-y e a r \\
\text { base flow } \\
\left(\mathrm{ft}^{3} / \mathrm{s}\right)\end{array}$ & $\begin{array}{c}\text { 50-year } \\
\text { base flow } \\
\left(\mathrm{ft}^{3} / \mathrm{s}\right)\end{array}$ \\
\hline 01411456 & 1989-2008 & 20 & 4.3 & 3.6 & 3.2 \\
\hline 01411500 & 1934-2008 & 75 & 93.4 & 82.6 & 76.2 \\
\hline 01412000 & 1933-1984 & 31 & 20.8 & 18.1 & 16.4 \\
\hline 01412800 & 1979-2008 & 14 & 22.6 & 21.1 & 20.2 \\
\hline 01413500 & 1938-2008 & 71 & 134 & 116 & 105 \\
\hline 01414000 & 1943-2008 & 31 & 29.1 & 26.2 & 24.4 \\
\hline 01414500 & 1938-2008 & 71 & 26.6 & 23.5 & 21.6 \\
\hline 01415000 & 1938-2008 & 71 & 24.8 & 21.8 & 20.0 \\
\hline 01417000 & ${ }^{2} 1956-2008$ & 53 & 35.5 & 25.4 & 20.3 \\
\hline 01417000 & ${ }^{3} 1943-1954$ & 12 & 318 & 296 & 283 \\
\hline 01417500 & ${ }^{2} 1956-2008$ & 42 & 127 & 112 & 104 \\
\hline 01417500 & ${ }^{3} 1936-1954$ & 19 & 367 & 334 & 313 \\
\hline 01420000 & 1926-1981 & 56 & 18.1 & 15.9 & 14.5 \\
\hline 01420500 & 1915-2008 & 94 & 247 & 214 & 193 \\
\hline 01421000 & ${ }^{2} 1956-2008$ & 53 & 459 & 415 & 389 \\
\hline 01421000 & ${ }^{3} 1915-1954$ & 40 & 683 & 581 & 518 \\
\hline 01421900 & 1938-2008 & 44 & 101 & 85.0 & 75.4 \\
\hline 01422500 & 1939-2008 & 43 & 38.4 & 33.5 & 30.5 \\
\hline 01423000 & 1952-2008 & 57 & 219 & 189 & 170 \\
\hline 0142400103 & 1954-2008 & 25 & 13.7 & 11.9 & 10.8 \\
\hline 01425000 & ${ }^{2} 1965-2008$ & 44 & 113 & 69.6 & 49.0 \\
\hline 01425000 & ${ }^{3} 1953-1963$ & 10 & 72.9 & 18.7 & 6.2 \\
\hline 01425675 & 1971-1981 & 11 & 4.4 & 4.0 & 3.8 \\
\hline 01426000 & 1942-1973 & 32 & 39.6 & 34.1 & 30.8 \\
\hline 01426500 & ${ }^{2} 1965-2008$ & 44 & 233 & 179 & 150 \\
\hline 01426500 & ${ }^{3} 1914-1963$ & 50 & 406 & 340 & 298 \\
\hline 01427500 & 1942-1982 & 41 & 65.0 & 56.0 & 50.6 \\
\hline 01427510 & $1977-2008$ & 32 & 1,040 & 938 & 876 \\
\hline 01428500 & 1942-2008 & 67 & 1,210 & 1,050 & 948 \\
\hline 01428750 & 1988-2008 & 21 & 30.4 & 25.4 & 22.4 \\
\hline 01429000 & ${ }^{2} 1962-2008$ & 47 & 44.6 & 38.0 & 34.1 \\
\hline 01429000 & ${ }^{3} 1946-1960$ & 15 & 48.4 & 42.3 & 38.5 \\
\hline 01429500 & ${ }^{2} 1961-2008$ & 48 & 42.2 & 35.9 & 32.2 \\
\hline 01429500 & ${ }^{3} 1945-1959$ & 15 & 44.8 & 40.1 & 37.2 \\
\hline 01430000 & ${ }^{2} 1961-2008$ & 31 & 110 & 93.0 & 82.6 \\
\hline 01430000 & ${ }^{3} 1950-1959$ & 10 & 130 & 120 & 113 \\
\hline 01430500 & 1924-1938 & 15 & 144 & 121 & 107 \\
\hline 01431000 & 1947-1960 & 14 & 52.3 & 48.9 & 46.9 \\
\hline 01431500 & ${ }^{2} 1961-2008$ & 48 & 178 & 152 & 136 \\
\hline 01431500 & ${ }^{3} 1910-1959$ & 28 & 177 & 156 & 144 \\
\hline 01432000 & ${ }^{3} 1911-1925$ & 15 & 206 & 192 & 183 \\
\hline 01433500 & 1941-1995 & 55 & 99.1 & 76.7 & 64.3 \\
\hline 01434000 & 1910-2008 & 99 & 2,080 & 1,810 & 1,640 \\
\hline 0143400680 & 1992-2008 & 17 & 15.0 & 13.7 & 12.9 \\
\hline 01434017 & 1993-2008 & 16 & 37.9 & 35.2 & 33.7 \\
\hline
\end{tabular}


Table 3. Selected base-flow statistics for streamgage locations in and near Pennsylvania.-Continued

[ft $\mathrm{ft}^{3} / \mathrm{s}$; cubic feet per second; - , statistic not computed]

\begin{tabular}{|c|c|c|c|c|c|}
\hline $\begin{array}{c}\text { Streamgage } \\
\text { number }\end{array}$ & $\begin{array}{c}\text { Period of } \\
\text { record used } \\
\text { in analysis }\end{array}$ & $\begin{array}{l}\text { Number of } \\
\text { years used } \\
\text { in analysis }\end{array}$ & $\begin{array}{c}10 \text {-year } \\
\text { base flow } \\
\left(\mathrm{ft}^{3} / \mathrm{s}\right)\end{array}$ & $\begin{array}{c}25 \text {-year } \\
\text { base flow } \\
\left(\mathrm{ft}^{3} / \mathrm{s}\right)\end{array}$ & $\begin{array}{c}\text { 50-year } \\
\text { base flow } \\
\left(\mathrm{ft}^{3} / \mathrm{s}\right)\end{array}$ \\
\hline 01434021 & $1992-2008$ & 17 & 1.0 & .9 & .8 \\
\hline 01434025 & 1985-2008 & 24 & 5.0 & 4.6 & 4.3 \\
\hline 01434498 & 1993-2008 & 16 & 52.8 & 48.8 & 46.3 \\
\hline 01435000 & 1939-2008 & 67 & 87.6 & 78.0 & 72.0 \\
\hline 01436000 & ${ }^{2} 1955-2008$ & 54 & 15.4 & 11.5 & 9.5 \\
\hline 01436000 & ${ }^{3} 1943-1953$ & 11 & 134 & 124 & 118 \\
\hline 01436500 & ${ }^{2} 1955-1993$ & 34 & 38.3 & 34.1 & 31.7 \\
\hline 01436500 & ${ }^{3} 1939-1953$ & 15 & 136 & 119 & 109 \\
\hline 01436690 & 1994-2008 & 15 & 106 & 97.1 & 91.9 \\
\hline 01437500 & ${ }^{2} 1955-2008$ & 54 & 194 & 172 & 159 \\
\hline 01437500 & ${ }^{3} 1939-1953$ & 15 & 267 & 230 & 209 \\
\hline 01438500 & 1941-2008 & 68 & 2,230 & 1,940 & 1,770 \\
\hline 01439500 & 1910-2008 & 99 & 111 & 95.4 & 85.8 \\
\hline 01440000 & 1925-2008 & 84 & 49.5 & 42.5 & 38.3 \\
\hline 01440200 & 1966-1995 & 30 & 2,110 & 1,650 & 1,380 \\
\hline 01440400 & 1959-2008 & 50 & 62.3 & 54.4 & 49.5 \\
\hline 01441000 & 1913-1938 & 26 & 56.9 & 48.7 & 43.7 \\
\hline 01442500 & $1952-2008$ & 57 & 237 & 206 & 188 \\
\hline 01443280 & 1994-2008 & 15 & 12.5 & 11.1 & 10.2 \\
\hline 01443500 & 1923-2008 & 84 & 87.5 & 73.5 & 65.3 \\
\hline 01443900 & 1968-2008 & 41 & 5.0 & 4.2 & 3.7 \\
\hline 01445000 & 1941-2008 & 27 & 25.2 & 20.9 & 18.4 \\
\hline 01445500 & 1923-2008 & 86 & 73.9 & 60.7 & 53.0 \\
\hline 01446000 & 1924-2008 & 42 & 24.0 & 20.5 & 18.5 \\
\hline 01446500 & 1924-2008 & 85 & 3,220 & 2,800 & 2,550 \\
\hline 01446600 & 1963-1978 & 16 & 4.7 & 3.7 & 3.2 \\
\hline 01447500 & 1945-2008 & 64 & 92.0 & 81.2 & 74.5 \\
\hline 01447680 & ${ }^{2} 1971-2008$ & 38 & 22.0 & 18.4 & 16.3 \\
\hline 01447720 & ${ }^{2} 1987-2008$ & 22 & 125 & 108 & 97.5 \\
\hline 01447720 & ${ }^{3} 1963-1985$ & 23 & 120 & 107 & 99.0 \\
\hline 01447800 & 1959-2008 & 50 & 255 & 221 & 200 \\
\hline 01448000 & 1918-1959 & 41 & 307 & 265 & 238 \\
\hline 01448500 & 1950-1996 & 47 & 2.4 & 2.1 & 1.9 \\
\hline 01449000 & 1984-2008 & 25 & 605 & 528 & 482 \\
\hline 01449360 & 1968-2008 & 41 & 53.5 & 46.2 & 41.8 \\
\hline 01449800 & 1969-2008 & 40 & 76.0 & 64.9 & 58.2 \\
\hline 01450500 & 1941-2008 & 68 & 70.7 & 61.9 & 56.6 \\
\hline 01451000 & ${ }^{2} 1962-2008$ & 47 & 847 & 739 & 675 \\
\hline 01451000 & ${ }^{3} 1948-1960$ & 13 & 1,000 & 913 & 856 \\
\hline 01451500 & 1947-2008 & 62 & 49.7 & 40.8 & 35.8 \\
\hline 01451650 & 1988-2008 & 21 & 68.2 & 59.6 & 54.7 \\
\hline 01451800 & 1967-2008 & 42 & 32.0 & 27.4 & 24.7 \\
\hline 01452000 & 1946-2008 & 63 & 38.5 & 31.7 & 27.7 \\
\hline 01452500 & 1950-2008 & 59 & 24.3 & 19.4 & 16.6 \\
\hline 01453000 & ${ }^{2} 1929-2008$ & 81 & 1,120 & 992 & 913 \\
\hline
\end{tabular}


Table 3. Selected base-flow statistics for streamgage locations in and near Pennsylvania._Continued

$\left[\mathrm{ft}^{3} / \mathrm{s}\right.$; cubic feet per second; - , statistic not computed]

\begin{tabular}{|c|c|c|c|c|c|}
\hline $\begin{array}{c}\text { Streamgage } \\
\text { number }\end{array}$ & $\begin{array}{l}\text { Period of } \\
\text { record used } \\
\text { in analysis }\end{array}$ & $\begin{array}{l}\text { Number of } \\
\text { years used } \\
\text { in analysis }\end{array}$ & $\begin{array}{c}10 \text {-year } \\
\text { base flow } \\
\left(\mathrm{ft}^{3} / \mathrm{s}\right)\end{array}$ & $\begin{array}{c}25 \text {-year } \\
\text { base flow } \\
\left(\mathrm{ft}^{3} / \mathrm{s}\right)\end{array}$ & $\begin{array}{c}\text { 50-year } \\
\text { base flow } \\
\left(\mathrm{ft}^{3} / \mathrm{s}\right)\end{array}$ \\
\hline 01453000 & ${ }^{3} 1904-1927$ & 18 & 1,030 & 944 & 890 \\
\hline 01454700 & 1968-2005 & 38 & 1,360 & 1,210 & 1,120 \\
\hline 01455500 & 1930-2008 & 52 & 21.0 & 15.8 & 12.9 \\
\hline 01457000 & 1905-2008 & 89 & 121 & 101 & 89.9 \\
\hline 01459500 & ${ }^{2} 1975-2008$ & 34 & 33.7 & 27.6 & 24.0 \\
\hline 01459500 & ${ }^{3} 1937-1973$ & 37 & 26.4 & 23.5 & 21.9 \\
\hline 01463500 & 1914-2008 & 95 & 5,130 & 4,530 & 4,170 \\
\hline 01463620 & 1974-2008 & 19 & 22.3 & 18.5 & 16.3 \\
\hline 01464000 & 1925-2008 & 84 & 52.2 & 43.9 & 39.0 \\
\hline 01464500 & $1942-2008$ & 65 & 60.4 & 54.4 & 50.7 \\
\hline 01464645 & 1987-2008 & 22 & 11.1 & 7.8 & 6.1 \\
\hline 01464720 & 1992-2008 & 17 & 13.2 & 11.3 & 10.2 \\
\hline 01465000 & 1886-1934 & 28 & 56.2 & 47.1 & 41.8 \\
\hline 01465500 & 1936-2008 & 73 & 89.5 & 75.3 & 67.0 \\
\hline 01465770 & 1966-1982 & 16 & 2.1 & 1.7 & 1.5 \\
\hline 01465798 & 1967-2008 & 42 & 7.1 & 6.2 & 5.6 \\
\hline 01465850 & 1963-2008 & 19 & 39.7 & 32.7 & 28.6 \\
\hline 01466500 & 1955-2008 & 54 & 1.4 & 1.2 & 1.1 \\
\hline 01467000 & 1923-2008 & 86 & 92.2 & 81.7 & 75.5 \\
\hline 01467042 & 1966-1981 & 16 & 24.0 & 20.0 & 17.7 \\
\hline 01467048 & $1967-2008$ & 42 & 31.1 & 27.9 & 26.1 \\
\hline 01467050 & 1967-1981 & 15 & 1.1 & 1.0 & .9 \\
\hline 01467081 & 1969-2008 & 38 & 6.5 & 5.8 & 5.4 \\
\hline 01467086 & 1967-1988 & 23 & 11.0 & 10.0 & 9.4 \\
\hline 01467087 & 1984-2008 & 25 & 10.3 & 9.3 & 8.7 \\
\hline 01467089 & 1968-1982 & 15 & 14.7 & 13.1 & 12.1 \\
\hline 01467150 & 1965-2008 & 44 & 12.4 & 10.7 & 9.7 \\
\hline 01467500 & 1945-1969 & 25 & 48.2 & 42.6 & 39.4 \\
\hline 01468500 & 1949-2008 & 40 & 140 & 121 & 110 \\
\hline 01469500 & 1921-2008 & 88 & 36.6 & 31.4 & 28.3 \\
\hline 01470500 & 1949-2008 & 60 & 303 & 264 & 241 \\
\hline 01470756 & 1974-1995 & 22 & 98.3 & 81.9 & 72.0 \\
\hline 01470779 & 1976-2008 & 33 & 56.9 & 48.1 & 42.8 \\
\hline 01470853 & 1984-2005 & 22 & 3.1 & 2.6 & 2.3 \\
\hline 01470960 & ${ }^{2} 1980-2008$ & 29 & 115 & 98.2 & 88.3 \\
\hline 01470960 & ${ }^{3} 1967-1978$ & 12 & 116 & 93.0 & 79.4 \\
\hline 01471000 & ${ }^{2} 1980-2008$ & 29 & 139 & 117 & 105 \\
\hline 01471000 & ${ }^{3} 1952-1978$ & 27 & 135 & 112 & 98.9 \\
\hline 01471510 & ${ }^{2} 1980-2008$ & 29 & 699 & 605 & 549 \\
\hline 01471510 & ${ }^{3} 1916-1930$ & 10 & 698 & 651 & 622 \\
\hline 01471875 & 1995-2008 & 14 & 40.6 & 36.5 & 34.2 \\
\hline 01471980 & 1976-2004 & 29 & 55.8 & 49.6 & 45.9 \\
\hline 01472000 & ${ }^{2} 1980-2008$ & 29 & 830 & 727 & 665 \\
\hline 01472000 & ${ }^{3} 1929-1978$ & 50 & 752 & 630 & 557 \\
\hline 01472157 & 1970-2008 & 39 & 36.7 & 31.7 & 28.8 \\
\hline
\end{tabular}


Table 3. Selected base-flow statistics for streamgage locations in and near Pennsylvania.-Continued

[ft $\mathrm{ft}^{3} / \mathrm{s}$; cubic feet per second; - , statistic not computed]

\begin{tabular}{|c|c|c|c|c|c|}
\hline $\begin{array}{c}\text { Streamgage } \\
\text { number }\end{array}$ & $\begin{array}{l}\text { Period of } \\
\text { record used } \\
\text { in analysis }{ }^{1}\end{array}$ & $\begin{array}{l}\text { Number of } \\
\text { years used } \\
\text { in analysis }\end{array}$ & $\begin{array}{c}10 \text {-year } \\
\text { base flow } \\
\left(\mathrm{ft}^{3} / \mathrm{s}\right)\end{array}$ & $\begin{array}{c}25 \text {-year } \\
\text { base flow } \\
\left(\mathrm{ft}^{3} / \mathrm{s}\right)\end{array}$ & $\begin{array}{c}50 \text {-year } \\
\text { base flow } \\
\left(\mathrm{ft}^{3} / \mathrm{s}\right)\end{array}$ \\
\hline 01472174 & 1969-1984 & 16 & 4.2 & 3.5 & 3.1 \\
\hline 01472198 & 1983-2008 & 26 & 26.8 & 23.8 & 22.1 \\
\hline 01472199 & 1983-2008 & 26 & 17.2 & 15.2 & 14.0 \\
\hline 01472500 & $1886-1913$ & 28 & 68.7 & 58.2 & 52.0 \\
\hline 01472620 & 1985-2008 & 24 & 2.6 & 1.0 & .5 \\
\hline 01472810 & 1992-2008 & 15 & 53.2 & 48.4 & 45.2 \\
\hline 01473000 & ${ }^{2} 1958-2008$ & 51 & 112 & 93.8 & 82.8 \\
\hline 01473000 & ${ }^{3} 1916-1956$ & 41 & 94.2 & 77.3 & 67.5 \\
\hline 01473120 & 1968-1994 & 27 & 17.3 & 14.3 & 12.6 \\
\hline 01473169 & 1984-2008 & 25 & 18.0 & 16.7 & 15.9 \\
\hline 01473500 & 1929-2008 & 9 & 767 & 578 & 474 \\
\hline 01473900 & 1963-2008 & 14 & 17.8 & 15.0 & 13.5 \\
\hline 01473950 & $1967-1981$ & 15 & 29.9 & 25.1 & 22.3 \\
\hline 01474000 & 1967-2008 & 42 & 41.4 & 36.6 & 33.7 \\
\hline 01474500 & 1933-2008 & 76 & 912 & 748 & 653 \\
\hline 01475000 & $1942-2006$ & 37 & 7.7 & 6.9 & 6.4 \\
\hline 01475300 & 1974-1997 & 24 & 3.9 & 3.5 & 3.3 \\
\hline 01475510 & 1965-1990 & 26 & 26.5 & 23.1 & 21.2 \\
\hline 01475530 & 1966-1981 & 19 & 2.9 & 2.6 & 2.3 \\
\hline 01475550 & 1965-1990 & 25 & 5.0 & 3.1 & 2.2 \\
\hline 01475850 & 1983-2008 & 26 & 10.6 & 9.8 & 9.3 \\
\hline 01476480 & 1988-2008 & 19 & 19.0 & 17.0 & 15.9 \\
\hline 01476500 & 1933-1954 & 22 & 21.5 & 18.8 & 17.1 \\
\hline 01477000 & 1933-2007 & 73 & 38.0 & 33.2 & 30.5 \\
\hline 01477120 & 1967-2008 & 42 & 20.1 & 18.5 & 17.5 \\
\hline 01477800 & $1947-2008$ & 62 & 1.9 & 1.6 & 1.4 \\
\hline 01478000 & 1944-2008 & 65 & 8.0 & 6.9 & 6.3 \\
\hline 01478500 & 1953-1979 & 23 & 34.8 & 29.4 & 26.4 \\
\hline 01479000 & 1933-2008 & 65 & 47.3 & 41.2 & 37.7 \\
\hline 01479820 & 1989-2008 & 20 & 18.4 & 16.7 & 15.8 \\
\hline 01480000 & 1944-2008 & 65 & 27.3 & 24.1 & 22.3 \\
\hline 01480015 & 1990-2008 & 19 & 31.1 & 27.7 & 25.9 \\
\hline 01480100 & $1965-1980$ & 16 & 2.7 & 2.4 & 2.2 \\
\hline 01480300 & 1962-2008 & 47 & 10.2 & 8.8 & 8.0 \\
\hline 01480500 & ${ }^{2} 1995-2008$ & 30 & 23.7 & 21.4 & 20.2 \\
\hline 01480500 & ${ }^{3} 1945-1993$ & 14 & 26.5 & 22.9 & 20.8 \\
\hline 01480617 & 1971-2008 & 38 & 36.5 & 32.2 & 29.7 \\
\hline 01480675 & 1968-2008 & 41 & 4.8 & 4.2 & 3.8 \\
\hline 01480685 & 1975-2008 & 34 & 13.7 & 11.8 & 10.6 \\
\hline 01480700 & ${ }^{2} 1975-2008$ & 34 & 41.0 & 36.0 & 33.1 \\
\hline 01480800 & 1960-1968 & 9 & 37.4 & 34.0 & 32.1 \\
\hline 01480870 & 1973-2008 & 36 & 66.8 & 59.1 & 54.7 \\
\hline 01481000 & ${ }^{3} 1913-1973$ & 51 & 164 & 141 & 128 \\
\hline 01481000 & ${ }^{2} 1975-2008$ & 34 & 189 & 169 & 158 \\
\hline 01481500 & ${ }^{2} 1975-2008$ & 34 & 214 & 190 & 177 \\
\hline
\end{tabular}


Table 3. Selected base-flow statistics for streamgage locations in and near Pennsylvania._Continued

$\left[\mathrm{ft}^{3} / \mathrm{s}\right.$; cubic feet per second; - , statistic not computed]

\begin{tabular}{|c|c|c|c|c|c|}
\hline $\begin{array}{c}\text { Streamgage } \\
\text { number }\end{array}$ & $\begin{array}{c}\text { Period of } \\
\text { record used } \\
\text { in analysis }\end{array}$ & $\begin{array}{l}\text { Number of } \\
\text { years used } \\
\text { in analysis }\end{array}$ & $\begin{array}{c}10 \text {-year } \\
\text { base flow } \\
\left(\mathrm{ft}^{3} / \mathrm{s}\right)\end{array}$ & $\begin{array}{c}25 \text {-year } \\
\text { base flow } \\
\left(\mathrm{ft}^{3} / \mathrm{s}\right)\end{array}$ & $\begin{array}{c}50 \text {-year } \\
\text { base flow } \\
\left(\mathrm{ft}^{3} / \mathrm{s}\right)\end{array}$ \\
\hline 01481500 & ${ }^{3} 1948-1973$ & 26 & 195 & 169 & 155 \\
\hline 01482500 & 1941-2008 & 47 & 7.6 & 6.5 & 5.9 \\
\hline 01483200 & 1958-2008 & 51 & 1.7 & 1.4 & 1.2 \\
\hline 01483700 & 1959-2008 & 50 & 11.8 & 9.5 & 8.2 \\
\hline 01484000 & 1933-2008 & 27 & 7.4 & 6.3 & 5.7 \\
\hline 01484100 & 1960-2008 & 49 & 1.8 & 1.5 & 1.4 \\
\hline 01484270 & 1973-2005 & 11 & 8.6 & 7.9 & 7.5 \\
\hline 01493000 & 1949-2008 & 56 & 11.6 & 10.0 & 9.2 \\
\hline 01493500 & 1953-2008 & 54 & 4.0 & 3.4 & 3.1 \\
\hline 01495000 & 1933-2008 & 76 & 28.5 & 24.5 & 22.2 \\
\hline 01496000 & 1950-1984 & 35 & 11.1 & 9.1 & 7.9 \\
\hline 01496200 & 1969-1992 & 24 & 4.8 & 4.2 & 3.9 \\
\hline 01496500 & 1931-1995 & 59 & 86.7 & 71.8 & 62.7 \\
\hline 01500000 & ${ }^{2} 1951-2008$ & 58 & 61.8 & 53.5 & 48.4 \\
\hline 01500500 & 1940-2008 & 57 & 664 & 569 & 512 \\
\hline 01502000 & 1940-1995 & 56 & 43.2 & 37.3 & 33.6 \\
\hline 01502500 & 1931-2008 & 68 & 350 & 296 & 263 \\
\hline 01503000 & 1914-2008 & 95 & 1,390 & 1,170 & 1,040 \\
\hline 01505000 & 1940-2008 & 60 & 174 & 151 & 137 \\
\hline 01508803 & 1968-1986 & 14 & 67.9 & 61.5 & 57.7 \\
\hline 01509000 & $1940-2008$ & 67 & 202 & 157 & 130 \\
\hline 01510000 & 1940-2008 & 63 & 117 & 104 & 95.8 \\
\hline 01512500 & 1914-2008 & 95 & 923 & 798 & 723 \\
\hline 01515000 & 1938-2008 & 65 & 2,800 & 2,450 & 2,250 \\
\hline 01516350 & 1978-2008 & 31 & 75.9 & 65.0 & 58.7 \\
\hline 01516500 & 1956-2008 & 53 & 3.8 & 3.0 & 2.5 \\
\hline 01518000 & ${ }^{2} 1979-2008$ & 30 & 133 & 114 & 104 \\
\hline 01518000 & ${ }^{3} 1940-1977$ & 38 & 99.9 & 83.7 & 74.0 \\
\hline 01518700 & ${ }^{2} 1981-2008$ & 28 & 155 & 132 & 119 \\
\hline 01518862 & 1985-2008 & 24 & 32.1 & 26.1 & 22.6 \\
\hline 01520000 & ${ }^{2} 1981-2008$ & 28 & 74.3 & 62.3 & 55.5 \\
\hline 01520000 & ${ }^{3} 1953-1978$ & 26 & 76.8 & 66.8 & 61.0 \\
\hline 01520500 & ${ }^{2} 1981-1995$ & 15 & 223 & 189 & 170 \\
\hline 01520500 & ${ }^{3} 1931-1979$ & 49 & 233 & 197 & 175 \\
\hline 01521500 & ${ }^{2} 1941-2008$ & 68 & 11.6 & 10.2 & 9.4 \\
\hline 01523500 & ${ }^{2} 1950-2008$ & 59 & 20.7 & 18.4 & 17.0 \\
\hline 01524500 & 1944-2008 & 65 & 55.3 & 48.6 & 44.7 \\
\hline 01526500 & ${ }^{2} 1980-2008$ & 29 & 405 & 346 & 312 \\
\hline 01526500 & ${ }^{3} 1920-1978$ & 59 & 329 & 209 & 145 \\
\hline 01527000 & 1952-1981 & 30 & 25.6 & 23.1 & 21.7 \\
\hline 01527500 & 1940-2008 & 12 & 84.2 & 70.9 & 63.0 \\
\hline 01528000 & 1938-1995 & 58 & 23.7 & 20.2 & 18.2 \\
\hline 01529000 & 1938-1982 & 45 & 11.1 & 9.6 & 8.7 \\
\hline 01529500 & 1920-2008 & 89 & 165 & 144 & 131 \\
\hline 01529950 & ${ }^{2} 1980-2008$ & 29 & 623 & 527 & 472 \\
\hline
\end{tabular}


Table 3. Selected base-flow statistics for streamgage locations in and near Pennsylvania.-Continued

[ft $\mathrm{ft}^{3} / \mathrm{s}$; cubic feet per second; - , statistic not computed]

\begin{tabular}{|c|c|c|c|c|c|}
\hline $\begin{array}{c}\text { Streamgage } \\
\text { number }\end{array}$ & $\begin{array}{c}\text { Period of } \\
\text { record used } \\
\text { in analysis }\end{array}$ & $\begin{array}{l}\text { Number of } \\
\text { years used } \\
\text { in analysis }\end{array}$ & $\begin{array}{c}10 \text {-year } \\
\text { base flow } \\
\left(\mathrm{ft}^{3} / \mathrm{s}\right)\end{array}$ & $\begin{array}{c}25 \text {-year } \\
\text { base flow } \\
\left(\mathrm{ft}^{3} / \mathrm{s}\right)\end{array}$ & $\begin{array}{c}50 \text {-year } \\
\text { base flow } \\
\left(\mathrm{ft}^{3} / \mathrm{s}\right)\end{array}$ \\
\hline 01530500 & 1940-2008 & 69 & 30.2 & 25.8 & 23.3 \\
\hline 01531000 & ${ }^{2} 1981-2008$ & 28 & - & - & - \\
\hline 01531000 & ${ }^{3} 1905-1979$ & 68 & 758 & 657 & 598 \\
\hline 01531500 & ${ }^{2} 1981-2008$ & 28 & 3,830 & 3,300 & 2,990 \\
\hline 01531500 & ${ }^{3} 1915-1979$ & 65 & 3,730 & 3,220 & 2,900 \\
\hline 01532000 & 1915-2008 & 94 & 89.5 & 75.0 & 66.4 \\
\hline 01532850 & 1967-1979 & 13 & 3.6 & 3.5 & 3.4 \\
\hline 01533400 & ${ }^{2} 1981-2008$ & 28 & 3,270 & 2,320 & 1,800 \\
\hline 01533500 & $1942-1958$ & 17 & 16.0 & 14.3 & 13.3 \\
\hline 01533950 & $1962-1978$ & 17 & 5.6 & 4.7 & 4.1 \\
\hline 01534000 & 1915-2008 & 94 & 183 & 155 & 139 \\
\hline 01534300 & 1960-2008 & 49 & 29.5 & 25.9 & 23.7 \\
\hline 01534500 & ${ }^{2} 1961-2008$ & 48 & 92.6 & 79.7 & 72.0 \\
\hline 01534500 & ${ }^{3} 1941-1959$ & 19 & 99.6 & 88.8 & 82.1 \\
\hline 01536000 & ${ }^{2} 1961-2008$ & 48 & 168 & 144 & 130 \\
\hline 01536000 & ${ }^{3} 1940-1959$ & 20 & 261 & 231 & 213 \\
\hline 01536500 & ${ }^{2} 1981-2008$ & 28 & 5,120 & 4,340 & 3,880 \\
\hline 01536500 & ${ }^{3} 1901-1979$ & 79 & 4,880 & 4,220 & 3,820 \\
\hline 01537000 & 1943-1993 & 51 & 15.5 & 13.5 & 12.3 \\
\hline 01537500 & 1941-1990 & 50 & 6.8 & 5.6 & 4.9 \\
\hline 01538000 & $1921-2008$ & 88 & 28.4 & 24.6 & 22.3 \\
\hline 01539000 & $1940-2008$ & 69 & 165 & 120 & 93.5 \\
\hline 01539500 & $1942-1958$ & 17 & 28.4 & 26.7 & 25.8 \\
\hline 01540200 & 1965-1981 & 17 & .9 & .8 & .6 \\
\hline 01540500 & ${ }^{2} 1981-2008$ & 28 & 4,750 & 3,480 & 2,780 \\
\hline 01540500 & ${ }^{3} 1906-1979$ & 74 & 5,230 & 4,190 & 3,560 \\
\hline 01541000 & 1915-2008 & 94 & 191 & 165 & 150 \\
\hline 01541200 & ${ }^{2} 1967-2008$ & 40 & 242 & 214 & 198 \\
\hline 01541200 & ${ }^{3} 1957-1965$ & 9 & 236 & 223 & 217 \\
\hline 01541303 & 1980-2008 & 29 & 329 & 289 & 264 \\
\hline 01541308 & 1969-1979 & 11 & 6.3 & 5.7 & 5.3 \\
\hline 01541500 & ${ }^{2} 1962-2008$ & 47 & 233 & 208 & 194 \\
\hline 01541500 & ${ }^{3} 1915-1960$ & 46 & 194 & 164 & 146 \\
\hline 01542000 & 1942-1993 & 52 & 54.8 & 49.7 & 46.7 \\
\hline 01542500 & ${ }^{2} 1967-2008$ & 33 & 1,100 & 1,030 & 980 \\
\hline 01542500 & ${ }^{3} 1941-1965$ & 20 & 985 & 902 & 853 \\
\hline 01542810 & 1966-2008 & 43 & 3.0 & 2.7 & 2.5 \\
\hline 01543000 & $1915-2008$ & 94 & 142 & 122 & 110 \\
\hline 01543500 & 1940-2008 & 69 & 474 & 415 & 380 \\
\hline 01544000 & ${ }^{2} 1957-2008$ & 52 & 120 & 102 & 91.5 \\
\hline 01544500 & 1942-2008 & 67 & 86.1 & 76.5 & 70.9 \\
\hline 01545000 & ${ }^{2} 1964-2008$ & 45 & 133 & 115 & 105 \\
\hline 01545500 & ${ }^{2} 1963-2008$ & 46 & 1,860 & 1,640 & 1,510 \\
\hline 01545500 & ${ }^{3} 1909-1961$ & 53 & 1,680 & 1,350 & 1,150 \\
\hline 01545600 & 1966-2008 & 43 & 28.0 & 24.0 & 21.6 \\
\hline
\end{tabular}


Table 3. Selected base-flow statistics for streamgage locations in and near Pennsylvania._Continued

$\left[\mathrm{ft}^{3} / \mathrm{s}\right.$; cubic feet per second; - , statistic not computed]

\begin{tabular}{|c|c|c|c|c|c|}
\hline $\begin{array}{c}\text { Streamgage } \\
\text { number }\end{array}$ & $\begin{array}{c}\text { Period of } \\
\text { record used } \\
\text { in analysis }\end{array}$ & $\begin{array}{l}\text { Number of } \\
\text { years used } \\
\text { in analysis }\end{array}$ & $\begin{array}{c}10 \text {-year } \\
\text { base flow } \\
\left(\mathrm{ft}^{3} / \mathrm{s}\right)\end{array}$ & $\begin{array}{c}25-y e a r \\
\text { base flow } \\
\left(\mathrm{ft}^{3} / \mathrm{s}\right)\end{array}$ & $\begin{array}{c}50 \text {-year } \\
\text { base flow } \\
\left(\mathrm{ft}^{3} / \mathrm{s}\right)\end{array}$ \\
\hline 01546000 & $1912-1934$ & 17 & 64.2 & 60.7 & 58.9 \\
\hline 01546400 & 1986-2008 & 23 & 34.3 & 30.1 & 27.7 \\
\hline 01546500 & 1942-2008 & 67 & 54.3 & 46.9 & 42.5 \\
\hline 01547100 & 1969-2008 & 40 & 135 & 105 & 87.3 \\
\hline 01547200 & 1957-2008 & 52 & 197 & 175 & 161 \\
\hline 01547500 & ${ }^{2} 1971-2008$ & 38 & 232 & 210 & 197 \\
\hline 01547500 & ${ }^{3} 1956-1969$ & 14 & 186 & 166 & 154 \\
\hline 01547700 & 1957-2008 & 52 & 20.6 & 17.8 & 16.2 \\
\hline 01547800 & 1971-1981 & 11 & 12.8 & 11.6 & 10.9 \\
\hline 01547950 & 1970-2008 & 39 & 116 & 99.0 & 88.7 \\
\hline 01548005 & ${ }^{2} 1971-2000$ & 25 & 392 & 336 & 303 \\
\hline 01548005 & ${ }^{3} 1912-1969$ & 58 & 300 & 231 & 189 \\
\hline 01548500 & 1920-2008 & 89 & 299 & 259 & 235 \\
\hline 01549000 & 1910-1920 & 11 & 400 & 354 & 326 \\
\hline 01549500 & $1942-2008$ & 67 & 20.7 & 18.2 & 16.7 \\
\hline 01549700 & 1959-2008 & 50 & 478 & 412 & 374 \\
\hline 01550000 & 1915-2008 & 94 & 111 & 95.8 & 86.8 \\
\hline 01551500 & ${ }^{2} 1963-2008$ & 46 & 3,380 & 3,020 & 2,810 \\
\hline 01551500 & ${ }^{3} 1901-1961$ & 61 & 3,000 & 2,570 & 2,320 \\
\hline 01552000 & 1927-2008 & 80 & 271 & 233 & 209 \\
\hline 01552500 & $1942-2008$ & 67 & 19.6 & 17.3 & 16.0 \\
\hline 01553130 & 1969-1981 & 13 & 5.0 & 4.5 & 4.2 \\
\hline 01553500 & ${ }^{3} 1941-1966$ & 26 & 4,020 & 3,710 & 3,540 \\
\hline 01553500 & ${ }^{2} 1968-2008$ & 41 & 4,210 & 3,740 & 3,470 \\
\hline 01553700 & 1981-2008 & 28 & 28.3 & 25.9 & 24.5 \\
\hline 01554000 & ${ }^{2} 1981-2008$ & 28 & 8,570 & 6,630 & 5,520 \\
\hline 01554000 & ${ }^{3} 1939-1979$ & 41 & 9,420 & 7,820 & 6,860 \\
\hline 01554500 & 1941-1993 & 53 & 49.4 & 43.6 & 40.0 \\
\hline 01555000 & 1931-2008 & 78 & 185 & 158 & 143 \\
\hline 01555500 & 1931-2008 & 78 & 80.1 & 66.6 & 58.6 \\
\hline 01556000 & 1918-2008 & 91 & 156 & 136 & 124 \\
\hline 01557500 & 1946-2008 & 63 & 31.7 & 28.0 & 25.8 \\
\hline 01558000 & 1940-2008 & 69 & 187 & 169 & 159 \\
\hline 01559000 & 1943-2008 & 66 & 489 & 437 & 407 \\
\hline 01559500 & 1931-1958 & 28 & 58.7 & 48.3 & 42.1 \\
\hline 01559700 & 1963-1978 & 16 & 2.0 & 1.7 & 1.5 \\
\hline 01560000 & 1941-2008 & 68 & 83.5 & 73.9 & 68.3 \\
\hline 01561000 & $1932-1958$ & 27 & 14.6 & 11.2 & 9.3 \\
\hline 01562000 & 1913-2008 & 96 & 310 & 263 & 235 \\
\hline 01562500 & 1931-1957 & 27 & 32.7 & 25.3 & 21.0 \\
\hline 01563200 & ${ }^{2} 1974-2008$ & 35 & 439 & 382 & 348 \\
\hline 01563200 & ${ }^{3} 1948-1972$ & 25 & 297 & 248 & 220 \\
\hline 01563500 & ${ }^{2} 1974-2008$ & 35 & 1,140 & 1,040 & 981 \\
\hline 01563500 & ${ }^{3} 1939-1972$ & 34 & 865 & 761 & 700 \\
\hline 01564500 & 1940-2008 & 69 & 72.5 & 59.5 & 52.0 \\
\hline
\end{tabular}


Table 3. Selected base-flow statistics for streamgage locations in and near Pennsylvania._Continued

[ft $\mathrm{ft}^{3} / \mathrm{s}$; cubic feet per second; - , statistic not computed]

\begin{tabular}{|c|c|c|c|c|c|}
\hline $\begin{array}{c}\text { Streamgage } \\
\text { number }\end{array}$ & $\begin{array}{l}\text { Period of } \\
\text { record used } \\
\text { in analysis }\end{array}$ & $\begin{array}{l}\text { Number of } \\
\text { years used } \\
\text { in analysis }\end{array}$ & $\begin{array}{c}10 \text {-year } \\
\text { base flow } \\
\left(\mathrm{ft}^{3} / \mathrm{s}\right)\end{array}$ & $\begin{array}{c}25 \text {-year } \\
\text { base flow } \\
\left(\mathrm{ft}^{3} / \mathrm{s}\right)\end{array}$ & $\begin{array}{c}50 \text {-year } \\
\text { base flow } \\
\left(\mathrm{ft}^{3} / \mathrm{s}\right)\end{array}$ \\
\hline 01565000 & $1941-2008$ & 37 & 104 & 92.5 & 86.1 \\
\hline 01565700 & 1965-1981 & 17 & 2.6 & 2.1 & 1.9 \\
\hline 01566000 & 1913-2008 & 52 & 86.6 & 68.0 & 56.9 \\
\hline 01566500 & $1932-1958$ & 27 & 26.3 & 22.8 & 20.8 \\
\hline 01567000 & ${ }^{2} 1974-2008$ & 35 & 1,650 & 1,490 & 1,390 \\
\hline 01567000 & ${ }^{3} 1901-1972$ & 72 & 1,450 & 1,230 & 1,100 \\
\hline 01567500 & 1955-2008 & 54 & 8.0 & 6.9 & 6.4 \\
\hline 01568000 & 1931-2008 & 78 & 103 & 84.1 & 73.2 \\
\hline 01568500 & ${ }^{2} 1943-1997$ & 55 & 5.7 & 4.3 & 3.6 \\
\hline 01569000 & 1939-1974 & 14 & 24.0 & 19.9 & 17.5 \\
\hline 01569800 & 1978-2008 & 31 & 28.5 & 25.3 & 23.4 \\
\hline 01570000 & ${ }^{3} 1913-1969$ & 35 & 201 & 156 & 129 \\
\hline 01570000 & ${ }^{2} 1971-2008$ & 38 & 231 & 194 & 172 \\
\hline 01570500 & ${ }^{3} 1901-1972$ & 72 & 12,400 & 10,600 & 8,310 \\
\hline 01570500 & ${ }^{2} 1974-2008$ & 35 & 12,800 & 9,840 & 9,290 \\
\hline 01571000 & 1941-1995 & 16 & 4.9 & 4.0 & 3.5 \\
\hline 01571500 & 1911-2008 & 62 & 164 & 146 & 135 \\
\hline 01572000 & 1921-1984 & 14 & 19.1 & 14.5 & 11.8 \\
\hline 01572025 & 1990-2008 & 17 & 93.7 & 79.1 & 70.3 \\
\hline 01572190 & 1990-2008 & 17 & 124 & 102 & 89.4 \\
\hline 01573000 & 1920-2008 & 89 & 190 & 156 & 136 \\
\hline 01573086 & 1965-1981 & 17 & 3.4 & 2.4 & 1.8 \\
\hline 01573160 & 1977-1994 & 18 & 56.5 & 48.4 & 43.6 \\
\hline 01573500 & 1939-1958 & 20 & 10.0 & 8.7 & 8.0 \\
\hline 01573560 & $1977-2008$ & 30 & 272 & 229 & 204 \\
\hline 01574000 & 1930-2008 & 79 & 144 & 113 & 94.9 \\
\hline 01574500 & ${ }^{2} 1968-2008$ & 41 & 36.8 & 30.7 & 27.1 \\
\hline 01574500 & ${ }^{3} 1930-1966$ & 34 & 27.9 & 23.4 & 20.8 \\
\hline 01575000 & ${ }^{2} 1973-1995$ & 23 & 38.8 & 31.9 & 28.1 \\
\hline 01575000 & ${ }^{3} 1929-1971$ & 43 & 31.8 & 23.5 & 19.1 \\
\hline 01575500 & ${ }^{2} 1948-1996$ & 49 & 77.6 & 62.7 & 54.4 \\
\hline 01576000 & ${ }^{3} 1933-1972$ & 40 & 13,200 & 11,000 & 9,770 \\
\hline 01576000 & ${ }^{2} 1974-2008$ & 35 & 14,200 & 11,800 & 10,400 \\
\hline 01576085 & 1984-1995 & 12 & 2.9 & 2.5 & 2.3 \\
\hline 01576500 & $1931-2008$ & 78 & 148 & 117 & 99.8 \\
\hline 01576754 & $1986-2008$ & 23 & 278 & 235 & 210 \\
\hline 01578310 & 1969-2008 & 40 & 11,500 & 9,730 & 8,750 \\
\hline 01578400 & 1964-1981 & 18 & 3.2 & 2.7 & 2.3 \\
\hline 01580000 & 1928-2008 & 81 & 57.4 & 48.5 & 43.4 \\
\hline 01581500 & $1946-2008$ & 28 & 3.3 & 2.9 & 2.7 \\
\hline 01581700 & 1969-2008 & 40 & 21.3 & 18.0 & 16.1 \\
\hline 01582000 & 1946-2008 & 63 & 32.5 & 27.8 & 25.2 \\
\hline 01582500 & $1985-2008$ & 24 & 106 & 92.4 & 84.8 \\
\hline 01583000 & 1949-1981 & 33 & 1.0 & .8 & .8 \\
\hline 01583100 & 1984-2008 & 15 & 7.3 & 6.4 & 5.9 \\
\hline
\end{tabular}


Table 3. Selected base-flow statistics for streamgage locations in and near Pennsylvania._Continued

$\left[\mathrm{ft}^{3} / \mathrm{s}\right.$; cubic feet per second; - , statistic not computed]

\begin{tabular}{|c|c|c|c|c|c|}
\hline $\begin{array}{c}\text { Streamgage } \\
\text { number }\end{array}$ & $\begin{array}{c}\text { Period of } \\
\text { record used } \\
\text { in analysis }{ }^{1}\end{array}$ & $\begin{array}{l}\text { Number of } \\
\text { years used } \\
\text { in analysis }\end{array}$ & $\begin{array}{c}10 \text {-year } \\
\text { base flow } \\
\left(\mathrm{ft}^{3} / \mathrm{s}\right)\end{array}$ & $\begin{array}{c}25 \text {-year } \\
\text { base flow } \\
\left(\mathrm{ft}^{3} / \mathrm{s}\right)\end{array}$ & $\begin{array}{c}50 \text {-year } \\
\text { base flow } \\
\left(\mathrm{ft}^{3} / \mathrm{s}\right)\end{array}$ \\
\hline 01583500 & 1946-2008 & 63 & 31.1 & 26.6 & 24.0 \\
\hline 01583600 & 1984-2008 & 25 & 13.5 & 12.0 & 11.1 \\
\hline 01584050 & 1977-2008 & 32 & 5.1 & 4.4 & 4.0 \\
\hline 01584500 & 1928-2008 & 52 & 19.3 & 16.1 & 14.3 \\
\hline 01585095 & 1994-2008 & 15 & .3 & .2 & .2 \\
\hline 01585100 & 1960-2008 & 45 & 2.6 & 2.3 & 2.1 \\
\hline 01585200 & 1959-2008 & 39 & .6 & .5 & .5 \\
\hline 01585300 & 1960-1989 & 28 & 1.1 & 1.1 & 1.0 \\
\hline 01585400 & 1960-1987 & 28 & .5 & .5 & .5 \\
\hline 01585500 & 1951-2008 & 58 & 1.0 & .8 & .6 \\
\hline 01586000 & $1947-2008$ & 61 & 27.2 & 23.7 & 21.6 \\
\hline 01586210 & 1984-2008 & 25 & 8.3 & 7.4 & 6.9 \\
\hline 01586610 & 1984-2008 & 25 & 15.7 & 13.8 & 12.8 \\
\hline 01587500 & 1950-1980 & 31 & 29.6 & 25.3 & 22.9 \\
\hline 01589000 & ${ }^{2} 1956-2004$ & 41 & 52.4 & 45.6 & 42.1 \\
\hline 01589000 & ${ }^{3} 1946-1954$ & 9 & 169 & 156 & 148 \\
\hline 01589100 & 1959-2008 & 40 & .9 & .8 & .8 \\
\hline 01589300 & 1958-2008 & 42 & 13.5 & 11.9 & 11.0 \\
\hline 01589330 & 1961-2008 & 36 & 1.3 & 1.1 & 1.0 \\
\hline 01589440 & 1967-2008 & 33 & 13.1 & 11.5 & 10.6 \\
\hline 01589500 & 1946-2008 & 31 & .8 & .4 & .2 \\
\hline 01594930 & 1982-2004 & 23 & 11.7 & 10.8 & 10.4 \\
\hline 01594936 & 1982-2007 & 26 & 1.9 & 1.6 & 1.5 \\
\hline 01594950 & 1988-2008 & 21 & 1.8 & 1.6 & 1.5 \\
\hline 01595000 & 1958-2008 & 51 & 72.9 & 64.3 & 58.9 \\
\hline 01595200 & 1963-2008 & 46 & 34.2 & 30.1 & 27.8 \\
\hline 01595300 & 1958-1982 & 25 & 27.0 & 23.0 & 20.6 \\
\hline 01595500 & 1951-2008 & 39 & 190 & 170 & 157 \\
\hline 01595800 & ${ }^{3} 1968-1981$ & 14 & 207 & 182 & 167 \\
\hline 01596500 & 1950-2008 & 59 & 26.1 & 23.1 & 21.2 \\
\hline 01597000 & 1950-1981 & 32 & 12.2 & 10.6 & 9.7 \\
\hline 01597500 & 1952-2008 & 57 & 57.1 & 50.1 & 46.2 \\
\hline 01598500 & ${ }^{2} 1952-2008$ & 57 & 285 & 250 & 230 \\
\hline 01599000 & 1907-2008 & 78 & 29.6 & 24.6 & 21.7 \\
\hline 01600000 & ${ }^{2} 1952-2008$ & 38 & 340 & 299 & 275 \\
\hline 01600000 & ${ }^{3} 1940-1950$ & 11 & 290 & 253 & 231 \\
\hline 01601000 & 1953-2008 & 20 & 63.7 & 54.9 & 49.7 \\
\hline 01601500 & 1907-2008 & 78 & 107 & 90.8 & 81.3 \\
\hline 01603000 & ${ }^{2} 1983-2008$ & 26 & 567 & 500 & 460 \\
\hline 01603000 & ${ }^{3} 1931-1981$ & 51 & 433 & 370 & 332 \\
\hline 01603500 & 1934-1982 & 49 & 13.3 & 11.3 & 10.2 \\
\hline 01604500 & 1940-2008 & 69 & 44.2 & 35.7 & 31.1 \\
\hline 01609000 & 1930-2008 & 22 & 44.1 & 33.0 & 26.8 \\
\hline 01610000 & 1940-2008 & 69 & 1,100 & 969 & 895 \\
\hline 01610155 & 1969-2008 & 18 & 22.2 & 16.9 & 14.1 \\
\hline
\end{tabular}


Table 3. Selected base-flow statistics for streamgage locations in and near Pennsylvania.-Continued

[ft $\mathrm{ft}^{3} / \mathrm{s}$; cubic feet per second; - , statistic not computed]

\begin{tabular}{|c|c|c|c|c|c|}
\hline $\begin{array}{c}\text { Streamgage } \\
\text { number }\end{array}$ & $\begin{array}{l}\text { Period of } \\
\text { record used } \\
\text { in analysis }{ }^{1}\end{array}$ & $\begin{array}{l}\text { Number of } \\
\text { years used } \\
\text { in analysis }\end{array}$ & $\begin{array}{c}10 \text {-year } \\
\text { base flow } \\
\left(\mathrm{ft}^{3} / \mathrm{s}\right)\end{array}$ & $\begin{array}{c}25 \text {-year } \\
\text { base flow } \\
\left(\mathrm{ft}^{3} / \mathrm{s}\right)\end{array}$ & $\begin{array}{c}50 \text {-year } \\
\text { base flow } \\
\left(\mathrm{ft}^{3} / \mathrm{s}\right)\end{array}$ \\
\hline 01611500 & 1924-2008 & 83 & 170 & 144 & 129 \\
\hline 01613000 & 1934-2008 & 75 & 1,340 & 1,180 & 1,080 \\
\hline 01613050 & 1967-2008 & 40 & 4.0 & 3.3 & 2.9 \\
\hline 01614000 & $1930-2008$ & 41 & 46.7 & 35.6 & 29.5 \\
\hline 01614090 & $1962-1981$ & 19 & 3.4 & 2.9 & 2.6 \\
\hline 01614500 & $1930-2008$ & 79 & 209 & 173 & 153 \\
\hline 01616500 & 1949-2008 & 60 & 92.9 & 81.2 & 74.7 \\
\hline 01617000 & 1950-2008 & 24 & 4.0 & 3.0 & 2.4 \\
\hline 01617800 & $1966-2008$ & 43 & 4.9 & 3.8 & 3.2 \\
\hline 01618000 & $1930-2004$ & 68 & 1,850 & 1,550 & 1,380 \\
\hline 01619000 & 1950-2008 & 19 & 41.9 & 25.2 & 17.0 \\
\hline 01619500 & 1901-2008 & 82 & 137 & 117 & 106 \\
\hline 01637500 & 1949-2008 & 60 & 25.6 & 21.3 & 18.9 \\
\hline 01639000 & 1944-2008 & 65 & 39.6 & 26.3 & 19.2 \\
\hline 01639140 & $1992-2001$ & 10 & 9.6 & 8.4 & 7.7 \\
\hline 01639500 & 1949-2008 & 60 & 41.8 & 35.5 & 31.8 \\
\hline 01640500 & 1933-1984 & 52 & 4.0 & 3.3 & 2.9 \\
\hline 01640965 & 1983-1994 & 12 & 1.2 & 1.0 & .9 \\
\hline 01640970 & 1983-1991 & 9 & 2.8 & 2.5 & 2.3 \\
\hline 01641500 & 1949-1984 & 36 & 6.1 & 5.2 & 4.6 \\
\hline 01643000 & 1931-2008 & 78 & 277 & 226 & 196 \\
\hline 01643500 & $1950-2008$ & 50 & 26.6 & 23.3 & 21.5 \\
\hline 03007800 & $1976-2008$ & 33 & 154 & 134 & 121 \\
\hline 03008000 & 1968-1979 & 12 & 4.7 & 4.3 & 4.0 \\
\hline 03009680 & 1976-1995 & 20 & 123 & 109 & 101 \\
\hline 03010500 & $1941-2008$ & 68 & 372 & 333 & 309 \\
\hline 03010655 & $1976-2008$ & 33 & 58.9 & 52.2 & 48.3 \\
\hline 03011020 & 1910-2008 & 99 & 1,040 & 911 & 835 \\
\hline 03011800 & 1967-2008 & 42 & 35.0 & 31.5 & 29.3 \\
\hline 03012550 & ${ }^{2} 1937-1965$ & 25 & 1,410 & 1,250 & 1,150 \\
\hline 03013000 & 1940-1993 & 54 & 205 & 178 & 162 \\
\hline 03014500 & 1936-2008 & 73 & 196 & 170 & 155 \\
\hline 03015000 & ${ }^{2} 1951-2008$ & 58 & 675 & 603 & 561 \\
\hline 03015000 & ${ }^{3} 1941-1949$ & 9 & 662 & 554 & 487 \\
\hline 03015280 & 1964-1978 & 15 & 10.6 & 9.8 & 9.2 \\
\hline 03015500 & 1911-2008 & 98 & 213 & 190 & 176 \\
\hline 03016000 & ${ }^{2} 1967-2008$ & 44 & 2,490 & 2,140 & 1,920 \\
\hline 03016000 & ${ }^{3} 1943-1965$ & 23 & 2,390 & 2,150 & 2,000 \\
\hline 03017500 & 1939-1979 & 41 & 185 & 166 & 154 \\
\hline 03020000 & $1942-1991$ & 50 & 307 & 273 & 252 \\
\hline 03020500 & 1934-2008 & 75 & 201 & 182 & 170 \\
\hline 03021350 & 1976-2008 & 33 & 74.5 & 66.7 & 61.9 \\
\hline 03021410 & 1976-1993 & 18 & 44.1 & 39.5 & 36.5 \\
\hline 03021500 & $1911-1971$ & 61 & 148 & 134 & 126 \\
\hline 03021520 & 1973-1991 & 19 & 251 & 237 & 229 \\
\hline
\end{tabular}


Table 3. Selected base-flow statistics for streamgage locations in and near Pennsylvania._Continued

$\left[\mathrm{ft}^{3} / \mathrm{s}\right.$; cubic feet per second; - , statistic not computed]

\begin{tabular}{|c|c|c|c|c|c|}
\hline $\begin{array}{c}\text { Streamgage } \\
\text { number }\end{array}$ & $\begin{array}{l}\text { Period of } \\
\text { record used } \\
\text { in analysis }\end{array}$ & $\begin{array}{l}\text { Number of } \\
\text { years used } \\
\text { in analysis }\end{array}$ & $\begin{array}{c}10 \text {-year } \\
\text { base flow } \\
\left(\mathrm{ft}^{3} / \mathrm{s}\right)\end{array}$ & $\begin{array}{c}25-y e a r \\
\text { base flow } \\
\left(\mathrm{ft}^{3} / \mathrm{s}\right)\end{array}$ & $\begin{array}{c}\text { 50-year } \\
\text { base flow } \\
\left(\mathrm{ft}^{3} / \mathrm{s}\right)\end{array}$ \\
\hline 03022540 & $1975-1995$ & 16 & 22.2 & 19.8 & 18.3 \\
\hline 03022554 & 1976-1991 & 16 & 34.6 & 30.5 & 28.0 \\
\hline 03023100 & 1990-2008 & 19 & 599 & 507 & 453 \\
\hline 03024000 & ${ }^{2} 1972-2008$ & 37 & 764 & 648 & 578 \\
\hline 03024000 & ${ }^{3} 1934-1970$ & 37 & 620 & 552 & 510 \\
\hline 03025000 & 1935-1980 & 46 & 112 & 98.3 & 90.0 \\
\hline 03025500 & ${ }^{2} 1967-2008$ & 42 & 3,880 & 3,010 & 2,510 \\
\hline 03025500 & ${ }^{3} 1916-1965$ & 50 & 3,230 & 2,480 & 2,030 \\
\hline 03026500 & 1953-2008 & 56 & 6.3 & 5.5 & 5.0 \\
\hline 03027500 & ${ }^{2} 1954-1991$ & 38 & 73.6 & 57.0 & 46.9 \\
\hline 03028000 & 1955-2008 & 54 & 52.4 & 46.3 & 42.6 \\
\hline 03028500 & ${ }^{2} 1954-1995$ & 42 & 192 & 167 & 152 \\
\hline 03029500 & ${ }^{2} 1954-2008$ & 55 & 647 & 588 & 552 \\
\hline 03029500 & ${ }^{3} 1940-1952$ & 13 & 577 & 502 & 457 \\
\hline 03030500 & 1949-2008 & 60 & 444 & 373 & 332 \\
\hline 03031500 & ${ }^{2} 1967-2008$ & 42 & 5,070 & 3,990 & 3,360 \\
\hline 03031500 & ${ }^{3} 1934-1965$ & 32 & 3,850 & 2,800 & 2,200 \\
\hline 03031950 & 1965-1981 & 17 & 5.7 & 5.2 & 4.9 \\
\hline 03032500 & 1920-2008 & 87 & 298 & 249 & 219 \\
\hline 03034000 & 1940-2008 & 69 & 113 & 98.7 & 90.0 \\
\hline 03034500 & 1941-2008 & 68 & 51.7 & 46.1 & 42.8 \\
\hline 03036000 & ${ }^{2} 1942-1991$ & 50 & 210 & 192 & 182 \\
\hline 03036500 & ${ }^{2} 1967-2008$ & 42 & 5,780 & 4,580 & 3,880 \\
\hline 03036500 & ${ }^{3} 1906-1965$ & 53 & 5,090 & 4,120 & 3,530 \\
\hline 03038000 & ${ }^{2} 1970-2008$ & 39 & 90.8 & 77.8 & 70.1 \\
\hline 03038000 & ${ }^{3} 1939-1968$ & 30 & 89.7 & 78.1 & 71.1 \\
\hline 03039000 & ${ }^{2} 1941-1991$ & 51 & 118 & 102 & 92.0 \\
\hline 03039000 & ${ }^{3} 1920-1939$ & 40 & 96.1 & 69.3 & 54.0 \\
\hline 03039200 & 1966-1978 & 13 & 2.8 & 2.6 & 2.5 \\
\hline 03039925 & 1986-1998 & 10 & 3.6 & 3.0 & 2.7 \\
\hline 03040000 & $1915-2008$ & 89 & 233 & 200 & 180 \\
\hline 03041000 & 1940-2006 & 56 & 120 & 103 & 92.5 \\
\hline 03041500 & 1940-2008 & 69 & 568 & 518 & 489 \\
\hline 03042000 & 1953-2008 & 56 & 150 & 133 & 123 \\
\hline 03042200 & 1962-1988 & 18 & 5.6 & 5.1 & 4.8 \\
\hline 03042280 & ${ }^{2} 1973-2008$ & 36 & 38.2 & 33.6 & 30.9 \\
\hline 03042500 & ${ }^{2} 1970-2008$ & 39 & 125 & 113 & 105 \\
\hline 03042500 & ${ }^{3} 1953-1968$ & 16 & 104 & 94.6 & 88.8 \\
\hline 03043000 & 1909-1951 & 43 & 209 & 179 & 161 \\
\hline 03044000 & ${ }^{2} 1953-1991$ & 39 & 869 & 772 & 712 \\
\hline 03044000 & ${ }^{3} 1941-1951$ & 11 & 1,060 & 1,000 & 974 \\
\hline 03045000 & 1941-2008 & 68 & 112 & 99.5 & 91.7 \\
\hline 03045500 & 1921-1940 & 17 & 128 & 104 & 89.1 \\
\hline 03047000 & $1943-1991$ & 49 & 158 & 140 & 129 \\
\hline 03047500 & 1909-1937 & 29 & 1,030 & 869 & 766 \\
\hline
\end{tabular}


Table 3. Selected base-flow statistics for streamgage locations in and near Pennsylvania._Continued

[ft $\mathrm{ft}^{3} / \mathrm{s}$; cubic feet per second; - , statistic not computed]

\begin{tabular}{|c|c|c|c|c|c|}
\hline $\begin{array}{c}\text { Streamgage } \\
\text { number }\end{array}$ & $\begin{array}{l}\text { Period of } \\
\text { record used } \\
\text { in analysis }\end{array}$ & $\begin{array}{l}\text { Number of } \\
\text { years used } \\
\text { in analysis }\end{array}$ & $\begin{array}{c}10 \text {-year } \\
\text { base flow } \\
\left(\mathrm{ft}^{3} / \mathrm{s}\right)\end{array}$ & $\begin{array}{c}\text { 25-year } \\
\text { base flow } \\
\left(\mathrm{ft}^{3} / \mathrm{s}\right)\end{array}$ & $\begin{array}{c}50 \text {-year } \\
\text { base flow } \\
\left(\mathrm{ft}^{3} / \mathrm{s}\right)\end{array}$ \\
\hline 03048500 & ${ }^{2} 1943-2008$ & 66 & 1,140 & 1,000 & 917 \\
\hline 03049000 & 1942-2008 & 67 & 67.8 & 60.2 & 55.8 \\
\hline 03049500 & ${ }^{2} 1967-2008$ & 42 & 6,910 & 5,360 & 4,460 \\
\hline 03049500 & ${ }^{3} 1940-1965$ & 26 & 6,120 & 4,770 & 3,980 \\
\hline 03049800 & 1964-2008 & 45 & 2.0 & 1.7 & 1.5 \\
\hline 03061500 & 1909-2008 & 83 & 40.3 & 34.9 & 31.7 \\
\hline 03062400 & 1966-2002 & 33 & 5.5 & 4.7 & 4.2 \\
\hline 03062500 & 1947-2008 & 28 & 32.8 & 28.0 & 25.1 \\
\hline 03065000 & $1942-2008$ & 64 & 251 & 224 & 208 \\
\hline 03066000 & 1923-2008 & 86 & 70.9 & 59.5 & 52.4 \\
\hline 03068800 & 1975-2008 & 17 & 181 & 167 & 159 \\
\hline 03069000 & $1912-1993$ & 67 & 206 & 188 & 177 \\
\hline 03069500 & 1914-2008 & 95 & 553 & 482 & 439 \\
\hline 03070000 & 1925-1996 & 72 & 750 & 650 & 588 \\
\hline 03070420 & 1979-1995 & 17 & .7 & .7 & .7 \\
\hline 03070500 & 1911-2008 & 94 & 136 & 113 & 99.5 \\
\hline 03072000 & $1942-2008$ & 67 & 71.4 & 61.4 & 55.7 \\
\hline 03072655 & $1940-2008$ & 69 & 2,190 & 1,860 & 1,670 \\
\hline 03072840 & 1970-1979 & 10 & 48.7 & 42.5 & 38.9 \\
\hline 03073000 & 1933-1995 & 63 & 50.1 & 41.9 & 37.1 \\
\hline 03074300 & 1969-1979 & 11 & 2.8 & 2.5 & 2.4 \\
\hline 03074500 & 1944-2008 & 65 & 44.3 & 39.9 & 37.3 \\
\hline 03075070 & $1935-2008$ & 74 & 2,610 & 2,260 & 2,050 \\
\hline 03075500 & 1943-2008 & 66 & 116 & 103 & 95.1 \\
\hline 03076500 & ${ }^{2} 1941-2008$ & 67 & 232 & 206 & 190 \\
\hline 03076600 & 1966-2008 & 43 & 36.2 & 31.8 & 29.1 \\
\hline 03077500 & 1945-1991 & 47 & 406 & - & 333 \\
\hline 03078000 & 1949-2008 & 60 & 49.4 & 44.3 & 41.2 \\
\hline 03079000 & $1922-2008$ & 87 & 234 & 208 & 193 \\
\hline 03080000 & $1920-2008$ & 89 & 104 & 91.9 & 84.5 \\
\hline 03081000 & $1942-2008$ & 67 & 866 & 756 & 690 \\
\hline 03082200 & 1963-1978 & 16 & 7.1 & 6.4 & 6.0 \\
\hline 03082500 & ${ }^{2} 1926-2008$ & 83 & 1,000 & 836 & 734 \\
\hline 03082500 & ${ }^{3} 1910-1924$ & 13 & 529 & 334 & 234 \\
\hline 03083000 & 1943-1979 & 37 & 2.2 & 2.0 & 1.8 \\
\hline 03083500 & ${ }^{2} 1926-2008$ & 74 & 1,300 & 1,170 & 1,090 \\
\hline 03084000 & 1951-1994 & 44 & 1.8 & 1.5 & 1.4 \\
\hline 03085000 & 1940-2004 & 65 & 4,340 & 3,800 & 3,480 \\
\hline 03085500 & 1921-2008 & 80 & 108 & 92.0 & 82.3 \\
\hline 03086000 & 1935-2008 & 74 & 13,600 & 12,100 & 11,200 \\
\hline 03086500 & 1943-1993 & 51 & 18.9 & 15.1 & 12.9 \\
\hline 03090500 & ${ }^{2} 1945-1992$ & 48 & 89.3 & 74.6 & 66.3 \\
\hline 03090500 & ${ }^{3} 1933-1942$ & 11 & 36.0 & 30.0 & 26.5 \\
\hline 03091500 & 1931-2008 & 78 & 94.9 & 74.3 & 62.8 \\
\hline 03092000 & $1943-1993$ & 51 & 3.6 & 2.9 & 2.5 \\
\hline
\end{tabular}


Table 3. Selected base-flow statistics for streamgage locations in and near Pennsylvania._Continued

$\left[\mathrm{ft}^{3} / \mathrm{s}\right.$; cubic feet per second; - , statistic not computed]

\begin{tabular}{|c|c|c|c|c|c|}
\hline $\begin{array}{c}\text { Streamgage } \\
\text { number }\end{array}$ & $\begin{array}{c}\text { Period of } \\
\text { record used } \\
\text { in analysis }\end{array}$ & $\begin{array}{l}\text { Number of } \\
\text { years used } \\
\text { in analysis }\end{array}$ & $\begin{array}{c}10 \text {-year } \\
\text { base flow } \\
\left(\mathrm{ft}^{3} / \mathrm{s}\right)\end{array}$ & $\begin{array}{c}25 \text {-year } \\
\text { base flow } \\
\left(\mathrm{ft}^{3} / \mathrm{s}\right)\end{array}$ & $\begin{array}{c}\text { 50-year } \\
\text { base flow } \\
\left(\mathrm{ft}^{3} / \mathrm{s}\right)\end{array}$ \\
\hline 03092090 & 1967-1993 & 27 & 8.8 & 7.7 & 7.1 \\
\hline 03092460 & 1971-1992 & 22 & 54.7 & 47.7 & 43.6 \\
\hline 03092500 & ${ }^{2} 1969-1982$ & 14 & 46.2 & 35.1 & 28.7 \\
\hline 03092500 & ${ }^{3} 1929-1966$ & 39 & 23.6 & 20.2 & 18.3 \\
\hline 03093000 & 1928-2008 & 77 & 30.9 & 25.5 & 22.3 \\
\hline 03094000 & 1942-2008 & 67 & 231 & 196 & 177 \\
\hline 03095500 & 1945-1992 & 27 & 35.8 & 30.1 & 26.9 \\
\hline 03097550 & 1989-2008 & 20 & 341 & 279 & 245 \\
\hline 03098000 & 1923-1982 & 60 & 267 & 218 & 190 \\
\hline 03098500 & 1945-2000 & 27 & 14.2 & 12.3 & 11.3 \\
\hline 03098600 & 1989-2008 & 20 & 387 & 318 & 280 \\
\hline 03099500 & 1945-2000 & 46 & 445 & 388 & 354 \\
\hline 03100000 & $1913-1922$ & 10 & 65.5 & 59.3 & 55.8 \\
\hline 03101500 & $1936-2008$ & 73 & 93.8 & 55.7 & 36.8 \\
\hline 03102000 & ${ }^{3} 1921-1932$ & 12 & 78.4 & 66.2 & 59.0 \\
\hline 03102500 & 1915-2008 & 90 & 47.7 & 41.3 & 37.5 \\
\hline 03102850 & 1967-2008 & 42 & 199 & 170 & 153 \\
\hline 03102950 & 1967-1994 & 28 & 38.3 & 33.6 & 30.8 \\
\hline 03103000 & 1915-1963 & 46 & 54.9 & 46.8 & 42.3 \\
\hline 03103500 & 1939-1991 & 53 & 282 & 229 & 197 \\
\hline 03104000 & ${ }^{3} 1911-1932$ & 22 & 212 & 180 & 161 \\
\hline 03104500 & ${ }^{3} 1911-1932$ & 20 & 269 & 224 & 197 \\
\hline 03104760 & 1970-1981 & 12 & .8 & .7 & .6 \\
\hline 03105500 & 1916-2008 & 77 & 880 & 734 & 652 \\
\hline 03106000 & 1921-2008 & 88 & 136 & 112 & 97.9 \\
\hline 03106300 & ${ }^{2} 1971-2008$ & 36 & 25.7 & 18.9 & 15.1 \\
\hline 03106500 & ${ }^{2} 1971-2008$ & 38 & 218 & 183 & 162 \\
\hline 03106500 & ${ }^{3} 1913-1969$ & 57 & 183 & 156 & 140 \\
\hline 03107500 & 1958-2008 & 51 & 1,410 & 1,200 & 1,070 \\
\hline 03108000 & ${ }^{2} 1958-2008$ & 51 & 68.3 & 58.3 & 52.5 \\
\hline 03108000 & ${ }^{3} 1943-1956$ & 14 & 77.2 & 67.9 & 62.2 \\
\hline 03109500 & 1917-2008 & 92 & 167 & 143 & 129 \\
\hline 03110000 & 1942-2008 & 67 & 54.6 & 46.2 & 41.3 \\
\hline 03111150 & $1962-1985$ & 19 & 3.1 & 2.5 & 2.1 \\
\hline 03111500 & 1943-2008 & 66 & 53.3 & 46.3 & 42.3 \\
\hline 03111534 & 1980-1995 & 16 & 17,600 & 15,800 & 14,700 \\
\hline 03111548 & 1983-2008 & 21 & 52.2 & 46.7 & 43.6 \\
\hline 03112000 & 1942-2008 & 67 & 87.2 & 74.6 & 67.4 \\
\hline 03114000 & 1928-2003 & 52 & 41.1 & 31.7 & 26.4 \\
\hline 04209000 & 1927-2008 & 68 & 95.0 & 83.3 & 76.4 \\
\hline 04212500 & 1926-1979 & 47 & 30.2 & 24.9 & 21.9 \\
\hline 04213000 & 1924-2008 & 70 & 70.2 & 59.8 & 53.6 \\
\hline 04213040 & 1971-1996 & 26 & 1.0 & .8 & .7 \\
\hline 04213075 & 1988-2008 & 21 & 2.3 & 1.9 & 1.6 \\
\hline 04221000 & $1957-2008$ & 37 & 137 & 120 & 110 \\
\hline
\end{tabular}


Table 3. Selected base-flow statistics for streamgage locations in and near Pennsylvania.-Continued

$\left[\mathrm{ft}^{3} / \mathrm{s}\right.$; cubic feet per second; - , statistic not computed]

\begin{tabular}{cccccc}
\hline $\begin{array}{c}\text { Streamgage } \\
\text { number }\end{array}$ & $\begin{array}{c}\text { Period of } \\
\text { record used } \\
\text { in analysis }\end{array}$ & $\begin{array}{c}\text { Number of } \\
\text { years used } \\
\text { in analysis }\end{array}$ & $\begin{array}{c}\text { 10-year } \\
\text { base flow } \\
\left.\mathbf{f t t}^{3} / \mathbf{s}\right)\end{array}$ & $\begin{array}{c}\text { 25-year } \\
\text { base flow } \\
\left.\mathbf{f t}^{3} / \mathbf{s}\right)\end{array}$ & $\begin{array}{c}\mathbf{5 0} \text {-year } \\
\text { base flow } \\
\left.\mathbf{f t t}^{3} / \mathbf{s}\right)\end{array}$ \\
\hline 04223000 & $1910-2008$ & 99 & 430 & 381 & 352 \\
04224775 & $1976-2008$ & 33 & 38.4 & 33.0 & 29.9 \\
04227000 & $1960-2008$ & 44 & 103 & 89.0 & 80.9 \\
\hline
\end{tabular}

${ }^{1}$ Climatic year is defined as 12-month period beginning April 1 and ending March 31. The climatic year is designated by the calendar year in which it ends.

${ }^{2}$ Period of record for post-regulation period.

${ }^{3}$ Period of record for pre-regulation period. 
Table 4. Selected flood-flow statistics for streamgage locations in and near Pennsylvania.

$\left[\mathrm{ft}^{3} / \mathrm{s}\right.$; cubic feet per second; --, statistic not computed]

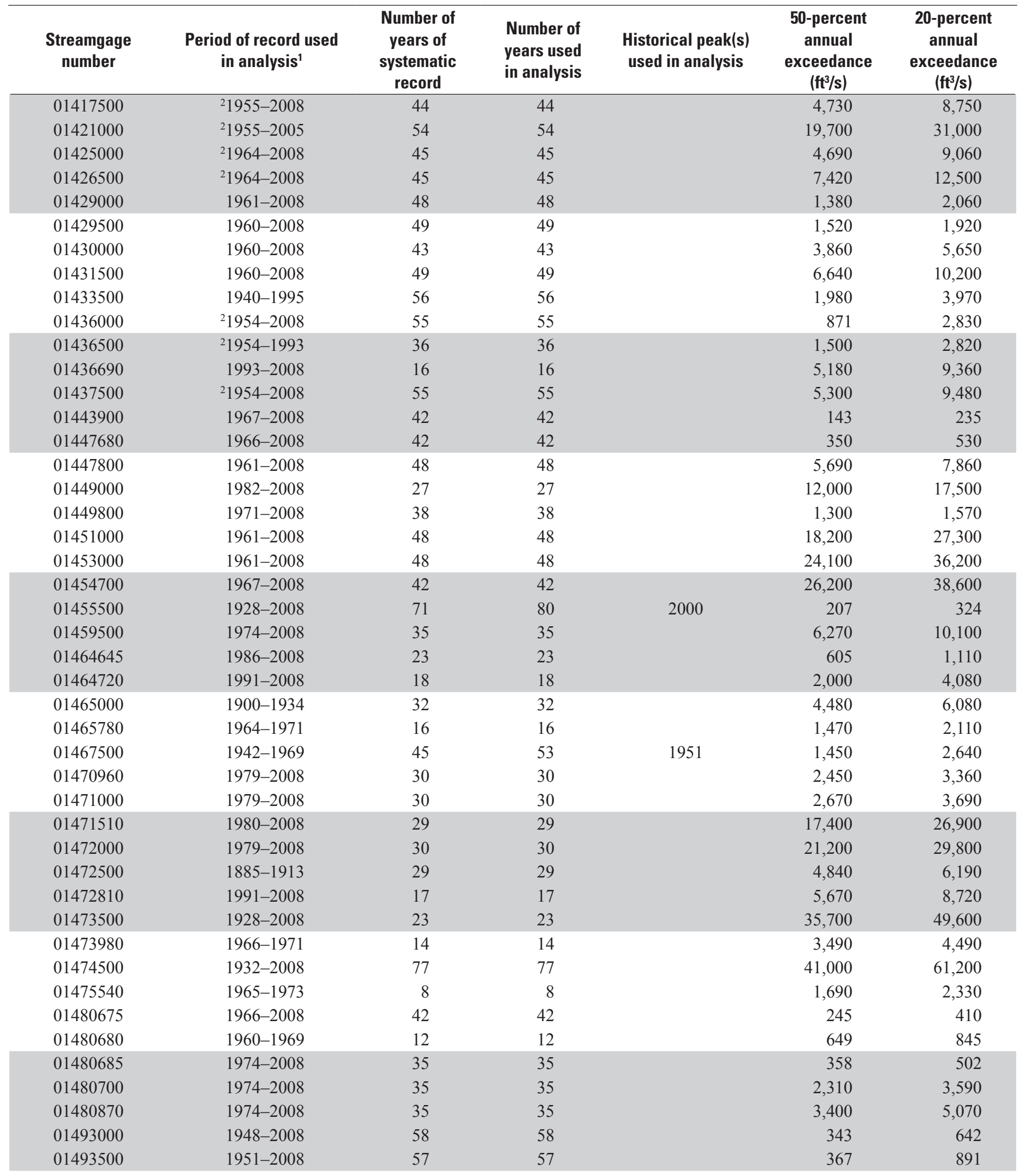


Table 4. Selected flood-flow statistics for streamgage locations in and near Pennsylvania.-Continued [ft $\mathrm{ft}^{3} / \mathrm{s}$; cubic feet per second; --, statistic not computed]

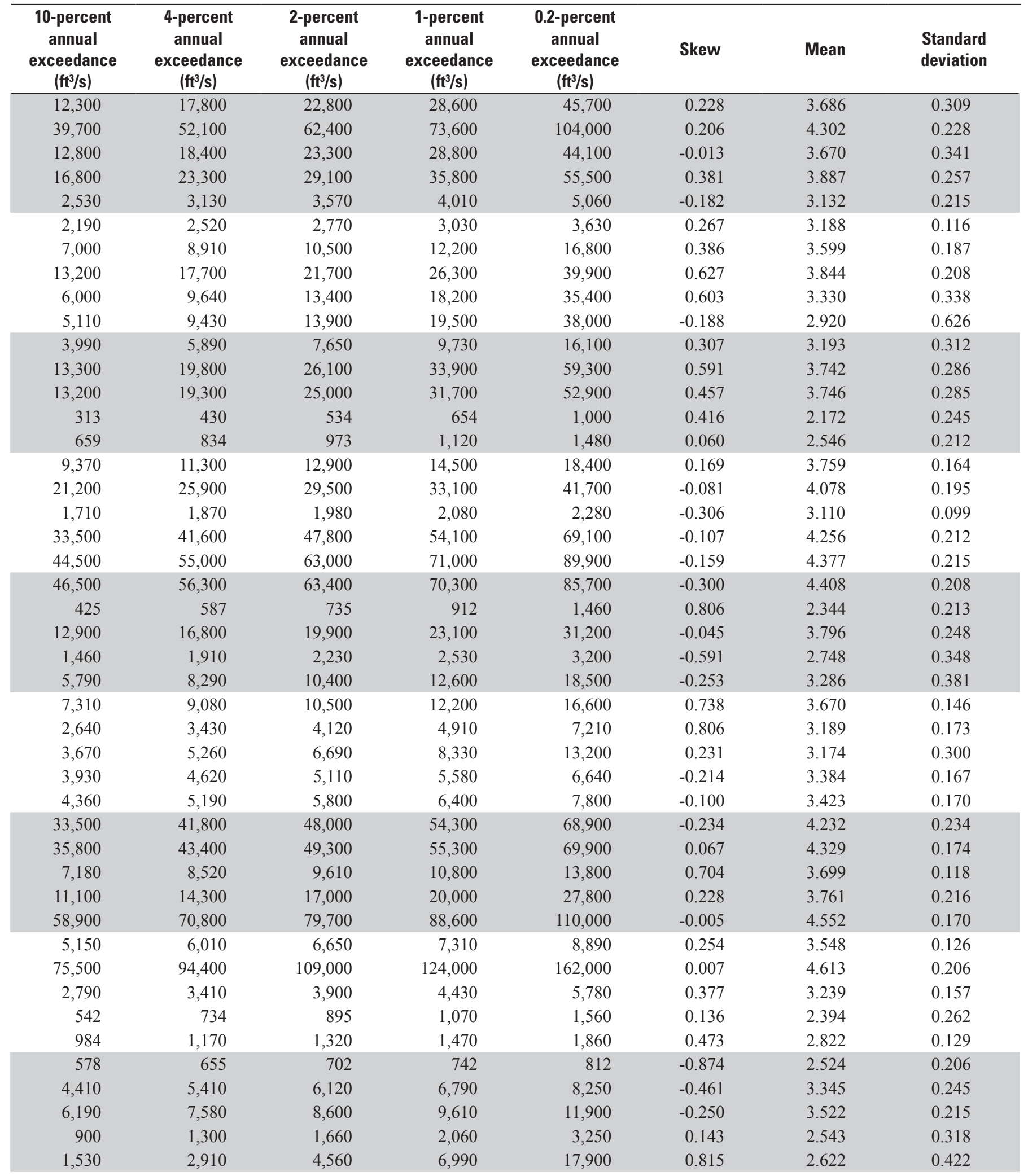


Table 4. Selected flood-flow statistics for streamgage locations in and near Pennsylvania._Continued

$\left[\mathrm{ft}^{3} / \mathrm{s}\right.$; cubic feet per second; --, statistic not computed]

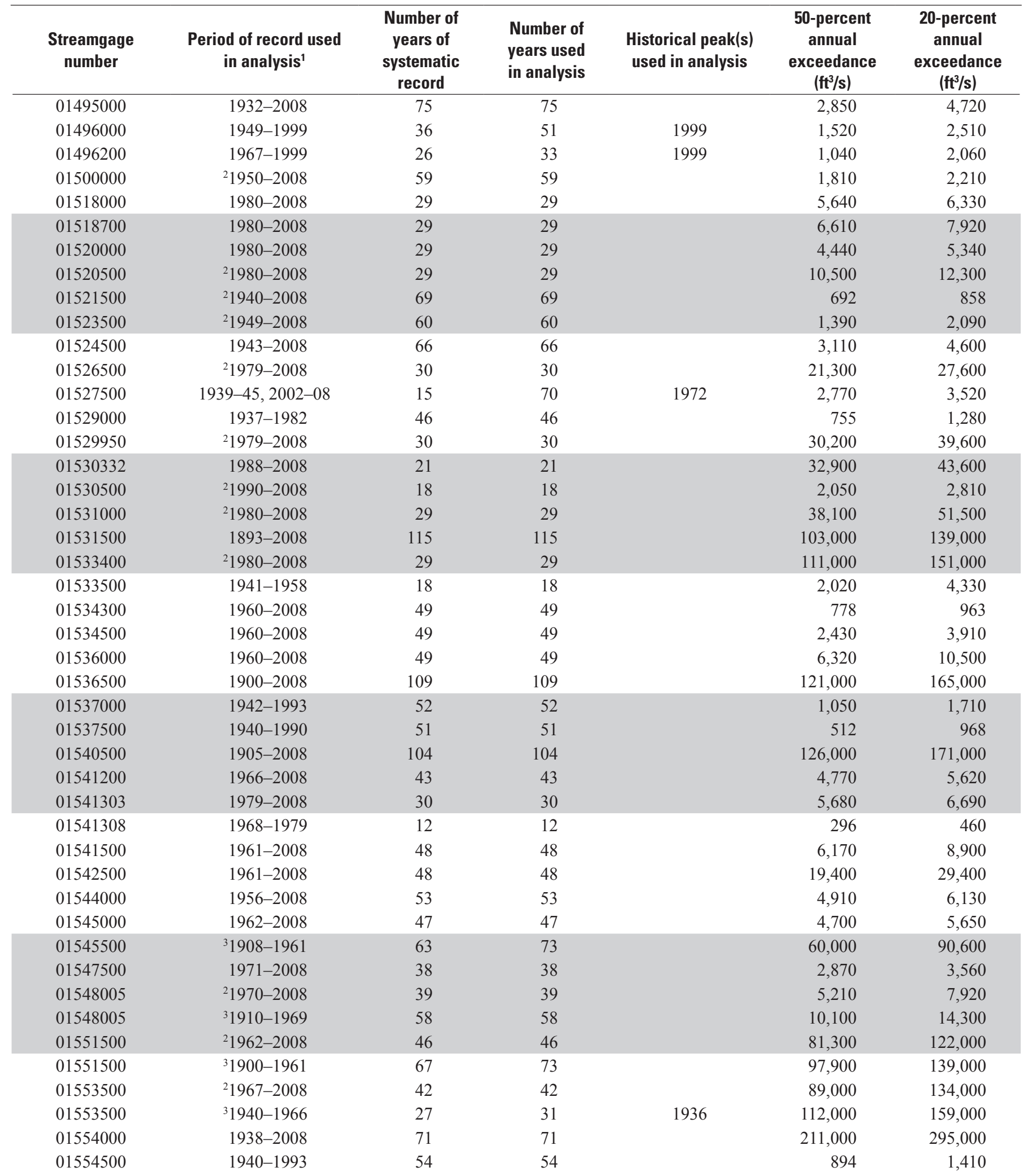


Table 4. Selected flood-flow statistics for streamgage locations in and near Pennsylvania. - Continued

[ $\mathrm{ft}^{3} / \mathrm{s}$; cubic feet per second; --, statistic not computed]

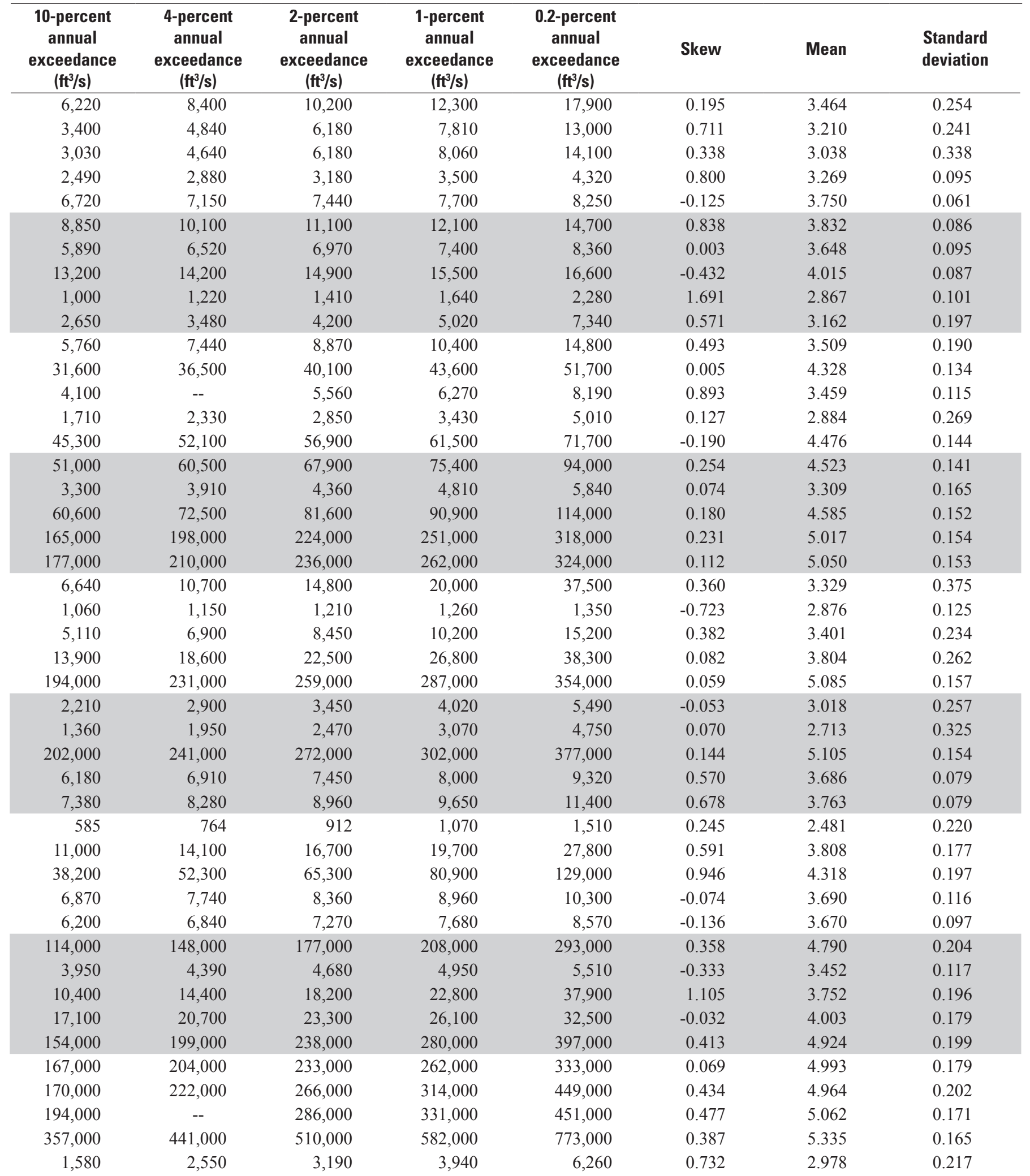


Table 4. Selected flood-flow statistics for streamgage locations in and near Pennsylvania._Continued

$\left[\mathrm{ft}^{3} / \mathrm{s}\right.$; cubic feet per second; --, statistic not computed]

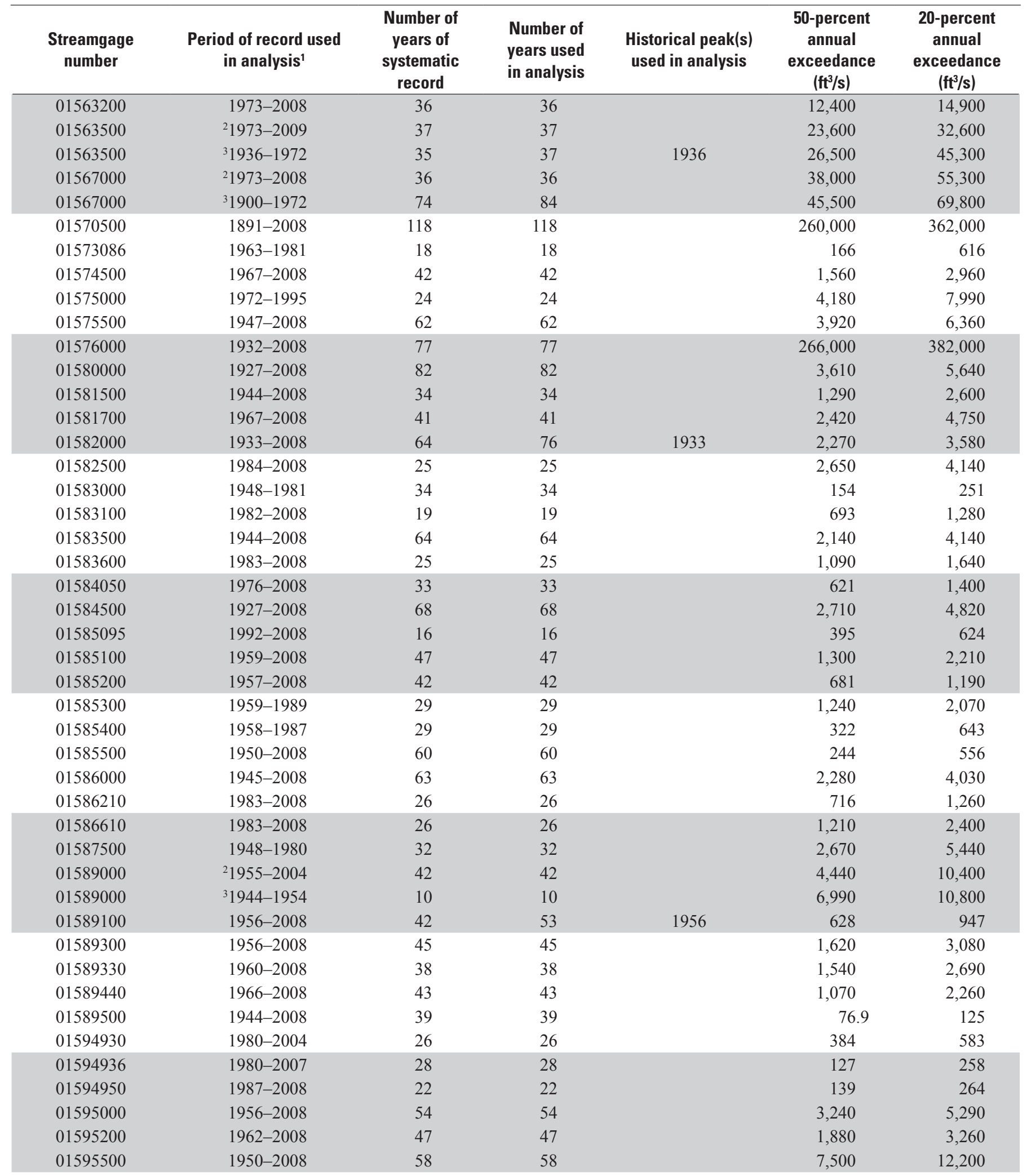


Table 4. Selected flood-flow statistics for streamgage locations in and near Pennsylvania.-Continued

[ft $\mathrm{ft}^{3} / \mathrm{s}$; cubic feet per second; --, statistic not computed]

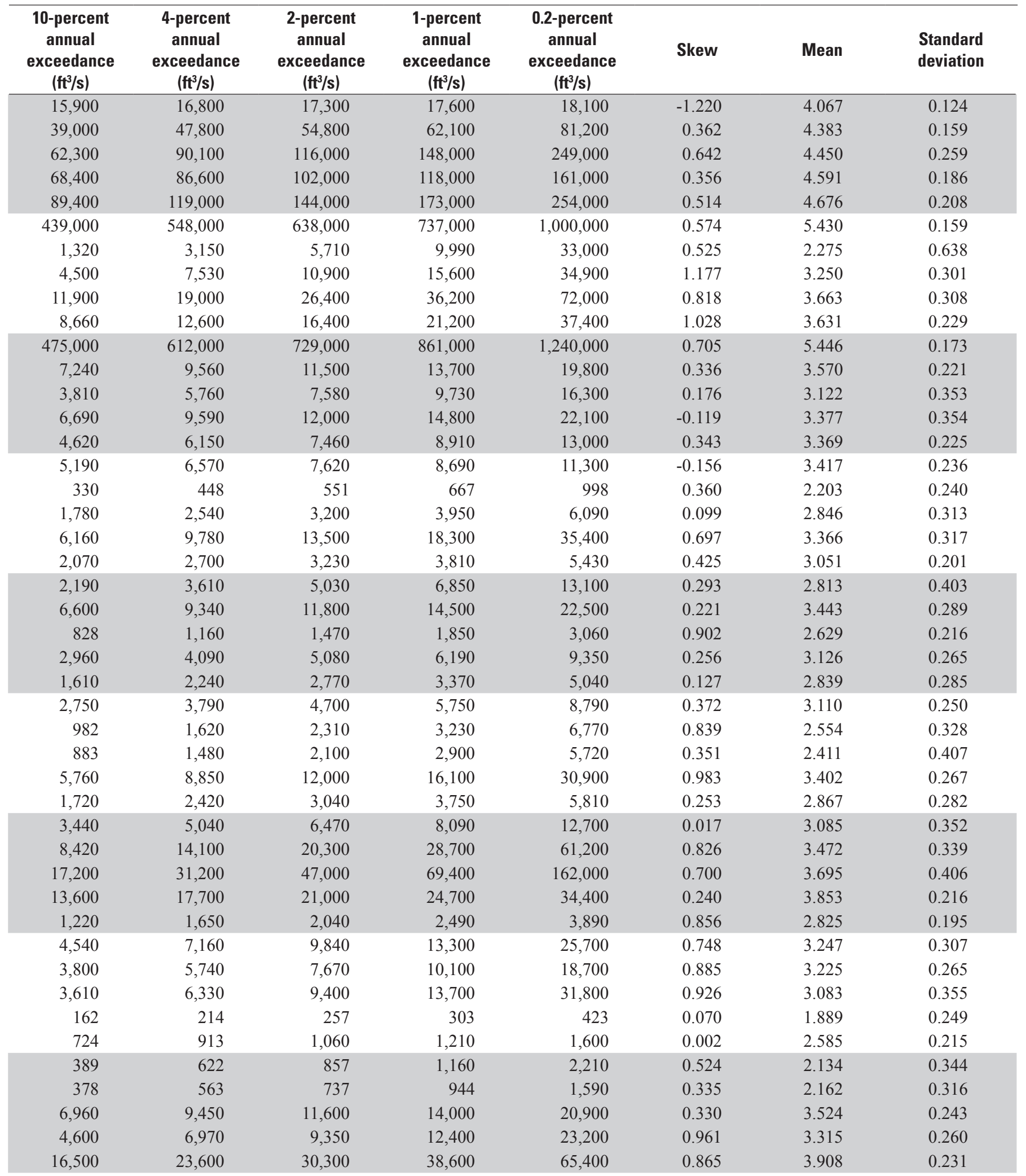


Table 4. Selected flood-flow statistics for streamgage locations in and near Pennsylvania._Continued

$\left[\mathrm{ft}^{3} / \mathrm{s}\right.$; cubic feet per second; --, statistic not computed]

\begin{tabular}{|c|c|c|c|c|c|c|}
\hline $\begin{array}{l}\text { Streamgage } \\
\text { number }\end{array}$ & $\begin{array}{l}\text { Period of record used } \\
\text { in analysis }{ }^{1}\end{array}$ & $\begin{array}{l}\text { Number of } \\
\text { years of } \\
\text { systematic } \\
\text { record }\end{array}$ & $\begin{array}{l}\text { Number of } \\
\text { years used } \\
\text { in analysis }\end{array}$ & $\begin{array}{l}\text { Historical peak(s) } \\
\text { used in analysis }\end{array}$ & $\begin{array}{c}\text { 50-percent } \\
\text { annual } \\
\text { exceedance } \\
\left(\mathrm{ft}^{3} / \mathrm{s}\right)\end{array}$ & $\begin{array}{c}20 \text {-percent } \\
\text { annual } \\
\text { exceedance } \\
\left(\mathrm{ft}^{3} / \mathrm{s}\right) \\
\end{array}$ \\
\hline 01595800 & ${ }^{2} 1982-2008$ & 26 & 26 & & 4,800 & 6,290 \\
\hline 01596500 & 1948-2008 & 60 & 60 & & 1,480 & 2,340 \\
\hline 01598500 & ${ }^{2} 1951-2008$ & 58 & 58 & & 7,710 & 11,800 \\
\hline 01599000 & 1905-2008 & 78 & 78 & & 1,860 & 3,000 \\
\hline 01600000 & ${ }^{2} 1951-2008$ & 57 & 57 & & 10,600 & 15,400 \\
\hline 01600000 & ${ }^{3} 1924-1950$ & 15 & 27 & 1924 & 16,500 & 28,500 \\
\hline 01601500 & $1930-2008$ & 79 & 79 & & 6,070 & 10,300 \\
\hline 01613000 & 1933-2008 & 76 & 76 & & 52,400 & 85,100 \\
\hline 01614500 & 1929-2008 & 80 & 85 & 1924 & 7,560 & 11,400 \\
\hline 01617800 & 1964-2008 & 44 & 44 & & 102 & 196 \\
\hline 01619000 & 1948-2008 & 23 & 23 & & 1,460 & 2,710 \\
\hline 01619500 & 1900-2008 & 81 & 81 & & 2,610 & 4,510 \\
\hline 01637500 & 1947-2008 & 61 & 61 & & 2,480 & 4,560 \\
\hline 01639000 & $1942-2008$ & 67 & 67 & & 8,470 & 11,700 \\
\hline 01639140 & 1990-2002 & 12 & 12 & & 1,860 & 2,910 \\
\hline 01639500 & 1948-2008 & 61 & 61 & & 3,260 & 5,460 \\
\hline 01640500 & 1932-1984 & 53 & 53 & & 377 & 875 \\
\hline 01643500 & 1948-2008 & 58 & 58 & & 2,460 & 4,710 \\
\hline 03012550 & ${ }^{2} 1966-1991$ & 19 & 19 & & 19,500 & 22,300 \\
\hline 03012550 & ${ }^{3} 1936-1965$ & 30 & 30 & & 34,100 & 44,900 \\
\hline 03014500 & 1935-2008 & 74 & 74 & & 1,300 & 1,590 \\
\hline 03015000 & ${ }^{2} 1950-2008$ & 59 & 59 & & 7,460 & 9,610 \\
\hline 03015000 & ${ }^{3} 1940-1949$ & 10 & 14 & 1936 & 9,260 & 12,700 \\
\hline 03016000 & ${ }^{2} 1966-2008$ & 41 & 41 & & 30,900 & 36,300 \\
\hline 03016000 & ${ }^{3} 1942-1965$ & 24 & 24 & & 51,500 & 70,800 \\
\hline 03020000 & 1941-1991 & 51 & 51 & & 6,590 & 8,340 \\
\hline 03021500 & 1910-1971 & 61 & 61 & & 6,900 & 10,200 \\
\hline 03021520 & 1972-1991 & 20 & 20 & & 2,380 & 3,100 \\
\hline 03022554 & 1975-1991 & 17 & 17 & & 608 & 775 \\
\hline 03023100 & 1989-2008 & 20 & 20 & & 10,600 & 13,200 \\
\hline 03024000 & 1971-2008 & 38 & 38 & & 12,300 & 14,900 \\
\hline 03025500 & ${ }^{2} 1966-2008$ & 44 & 44 & & 57,300 & 70,200 \\
\hline
\end{tabular}


Table 4. Selected flood-flow statistics for streamgage locations in and near Pennsylvania.-Continued

$\left[\mathrm{ft}^{3} / \mathrm{s}\right.$; cubic feet per second; --, statistic not computed]

\begin{tabular}{|c|c|c|c|c|c|c|c|}
\hline $\begin{array}{l}\text { 10-percent } \\
\text { annual } \\
\text { exceedance } \\
\left(\mathrm{ft}^{3} / \mathrm{s}\right)\end{array}$ & $\begin{array}{c}\text { 4-percent } \\
\text { annual } \\
\text { exceedance } \\
\left(\mathrm{ft}^{3} / \mathrm{s}\right)\end{array}$ & $\begin{array}{c}\text { 2-percent } \\
\text { annual } \\
\text { exceedance } \\
\left(\mathrm{ft}^{3} / \mathrm{s}\right)\end{array}$ & $\begin{array}{l}\text { 1-percent } \\
\text { annual } \\
\text { exceedance } \\
\left(\mathrm{ft}^{3} / \mathrm{s}\right)\end{array}$ & $\begin{array}{c}\text { 0.2-percent } \\
\text { annual } \\
\text { exceedance } \\
\left(\mathrm{ft}^{3} / \mathrm{s}\right)\end{array}$ & Skew & Mean & $\begin{array}{l}\text { Standard } \\
\text { deviation }\end{array}$ \\
\hline 7,090 & 7,930 & 8,460 & 8,920 & 9,780 & -0.691 & 3.663 & 0.158 \\
\hline 3,060 & 4,160 & 5,140 & 6,270 & 9,630 & 0.588 & 3.193 & 0.220 \\
\hline 15,100 & 20,300 & 25,000 & 30,400 & 46,400 & 0.712 & 3.911 & 0.202 \\
\hline 3,940 & 5,370 & 6,620 & 8,060 & 12,200 & 0.460 & 3.288 & 0.232 \\
\hline 18,700 & 23,000 & 26,200 & 29,500 & 37,300 & -0.059 & 4.023 & 0.196 \\
\hline 38,900 & 55,200 & 70,000 & 87,300 & 139,000 & 0.407 & 4.235 & 0.270 \\
\hline 14,200 & 20,600 & 26,800 & 34,400 & 59,300 & 0.757 & 3.815 & 0.252 \\
\hline 33,200 & 47,000 & 58,900 & 72,200 & 109,000 & 0.033 & 4.114 & 0.317 \\
\hline 113,000 & 156,000 & 194,000 & 239,000 & 372,000 & 0.532 & 4.740 & 0.236 \\
\hline 14,400 & 18,700 & 22,200 & 26,200 & 36,900 & 0.367 & 3.891 & 0.203 \\
\hline 277 & 401 & 510 & 634 & 986 & 0.043 & 2.011 & 0.335 \\
\hline 3,780 & 5,430 & 6,880 & 8,530 & 13,300 & 0.116 & 3.170 & 0.316 \\
\hline 6,110 & 8,570 & 10,700 & 13,200 & 20,500 & 0.297 & 3.430 & 0.272 \\
\hline 6,340 & 9,100 & 11,500 & 14,300 & 22,400 & 0.163 & 3.402 & 0.308 \\
\hline 14,000 & 17,100 & 19,600 & 22,200 & 28,800 & 0.337 & 3.937 & 0.159 \\
\hline 3,830 & 5,320 & 6,700 & 8,340 & 13,500 & 0.854 & 3.299 & 0.213 \\
\hline 7,550 & 11,100 & 14,700 & 19,100 & 34,300 & 0.968 & 3.552 & 0.243 \\
\hline 1,420 & 2,440 & 3,520 & 4,970 & 10,300 & 0.436 & 2.606 & 0.413 \\
\hline 7,060 & 11,500 & 16,200 & 22,500 & 46,400 & 0.930 & 3.438 & 0.307 \\
\hline 23,700 & 25,100 & 26,000 & 26,700 & 28,000 & -0.647 & 4.282 & 0.078 \\
\hline 51,200 & -- & 63,100 & 67,500 & 76,800 & -0.401 & 4.523 & 0.151 \\
\hline 1,780 & 2,010 & 2,180 & 2,350 & 2,740 & 0.197 & 3.117 & 0.103 \\
\hline 11,000 & 12,700 & 14,000 & 15,300 & 18,300 & 0.113 & 3.875 & 0.129 \\
\hline 14,900 & 17,500 & 19,400 & 21,200 & 25,200 & -0.245 & 3.960 & 0.171 \\
\hline 39,700 & 43,800 & 46,700 & 49,600 & 56,100 & 0.215 & 4.493 & 0.082 \\
\hline 82,800 & 97,200 & 107,000 & 117,000 & 139,000 & -0.258 & 4.705 & 0.171 \\
\hline 9,440 & 10,800 & 11,700 & 12,600 & 14,800 & 0.004 & 3.819 & 0.121 \\
\hline 12,600 & 15,700 & 18,100 & 20,600 & 26,800 & 0.030 & 3.840 & 0.202 \\
\hline 3,540 & 4,040 & 4,390 & 4,710 & 5,420 & -0.269 & 3.371 & 0.142 \\
\hline 878 & 1,000 & 1,090 & 1,170 & 1,360 & -0.081 & 2.782 & 0.127 \\
\hline 14,800 & 16,600 & 17,800 & 19,000 & 21,500 & -0.208 & 4.020 & 0.119 \\
\hline 16,300 & 17,800 & 18,800 & 19,700 & 21,400 & -0.485 & 4.083 & 0.107 \\
\hline 77,600 & 86,000 & 91,600 & 96,800 & 108,000 & -0.268 & 4.753 & 0.109 \\
\hline
\end{tabular}


Table 4. Selected flood-flow statistics for streamgage locations in and near Pennsylvania.-Continued

$\left[\mathrm{ft}^{3} / \mathrm{s}\right.$; cubic feet per second; --, statistic not computed]

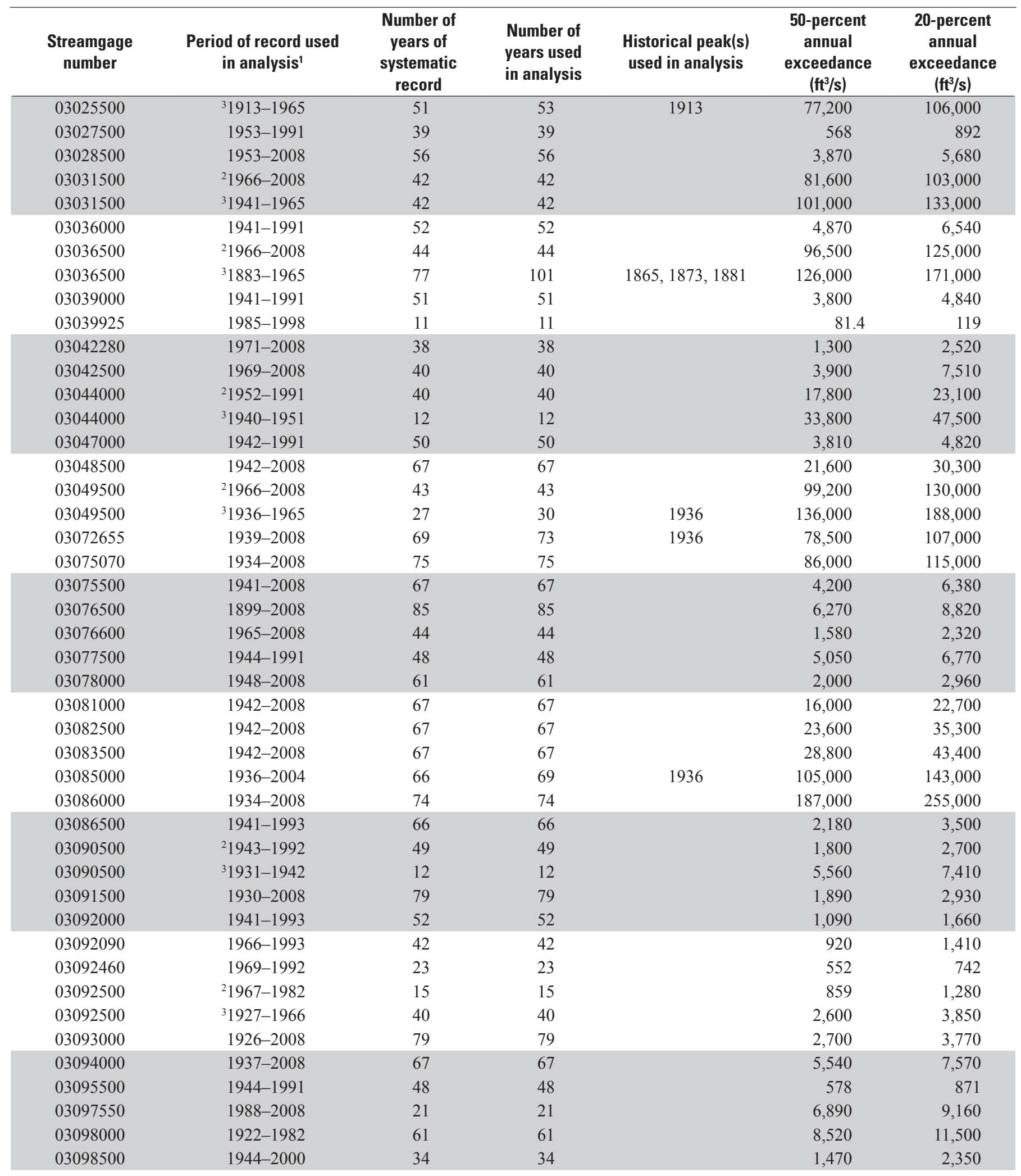


Table 4. Selected flood-flow statistics for streamgage locations in and near Pennsylvania.-Continued [ $\mathrm{ft}^{3} / \mathrm{s}$; cubic feet per second; --, statistic not computed]

\begin{tabular}{|c|c|c|c|c|c|c|c|}
\hline $\begin{array}{l}\text { 10-percent } \\
\text { annual } \\
\text { exceedance } \\
\left(\mathrm{ft}^{3} / \mathrm{s}\right)\end{array}$ & $\begin{array}{c}\text { 4-percent } \\
\text { annual } \\
\text { exceedance } \\
\left(\mathrm{ft}^{3} / \mathrm{s}\right)\end{array}$ & $\begin{array}{c}\text { 2-percent } \\
\text { annual } \\
\text { exceedance } \\
\left(\mathrm{ft}^{3} / \mathrm{s}\right)\end{array}$ & $\begin{array}{c}\text { 1-percent } \\
\text { annual } \\
\text { exceedance } \\
\left(\mathrm{ft}^{3} / \mathrm{s}\right)\end{array}$ & $\begin{array}{c}0.2 \text {-percent } \\
\text { annual } \\
\text { exceedance } \\
\left(\mathrm{ft}^{3} / \mathrm{s}\right)\end{array}$ & Skew & Mean & $\begin{array}{l}\text { Standard } \\
\text { deviation }\end{array}$ \\
\hline 125,000 & 148,000 & 166,000 & 183,000 & 224,000 & -0.036 & 4.887 & 0.163 \\
\hline 1,180 & 1,630 & 2,040 & 2,540 & 4,080 & 0.796 & 2.782 & 0.215 \\
\hline 7,050 & 9,000 & 10,600 & 12,400 & 17,100 & 0.398 & 3.601 & 0.188 \\
\hline 117,000 & 133,000 & 145,000 & 156,000 & 181,000 & -0.029 & 4.911 & 0.122 \\
\hline 153,000 & 176,000 & 192,000 & 207,000 & 241,000 & -0.256 & 4.997 & 0.150 \\
\hline 7,750 & 9,390 & 10,700 & 12,100 & 15,800 & 0.492 & 3.700 & 0.143 \\
\hline 143,000 & 166,000 & 182,000 & 198,000 & 236,000 & 0.056 & 4.986 & 0.132 \\
\hline 201,000 & 238,000 & 265,000 & 292,000 & 356,000 & -0.020 & 5.101 & 0.158 \\
\hline 5,440 & 6,130 & 6,590 & 7,020 & 7,920 & -0.331 & 3.573 & 0.132 \\
\hline 148 & 188 & 221 & 256 & 351 & 0.351 & 1.922 & 0.189 \\
\hline 3,800 & 6,180 & 8,710 & 12,100 & 24,700 & 0.857 & 3.158 & 0.315 \\
\hline 11,200 & 18,100 & 25,400 & 35,100 & 71,200 & 0.859 & 3.635 & 0.311 \\
\hline 26,300 & 30,200 & 33,000 & 35,600 & 41,500 & -0.131 & 4.248 & 0.136 \\
\hline 56,800 & 68,900 & 78,000 & 87,200 & 110,000 & 0.024 & 4.529 & 0.176 \\
\hline 5,430 & 6,150 & 6,660 & 7,150 & 8,230 & -0.111 & 3.579 & 0.123 \\
\hline 36,600 & 45,200 & 52,100 & 59,400 & 78,500 & 0.348 & 4.345 & 0.167 \\
\hline 151,000 & 177,000 & 197,000 & 217,000 & 264,000 & 0.154 & 5.000 & 0.138 \\
\hline 225,000 & 274,000 & 312,000 & 351,000 & 450,000 & 0.226 & 5.140 & 0.163 \\
\hline 126,000 & -- & 170,000 & 189,000 & 234,000 & 0.096 & 4.897 & 0.158 \\
\hline 133,000 & -- & 171,000 & 186,000 & 219,000 & -0.178 & 4.930 & 0.154 \\
\hline 8,050 & 10,400 & 12,400 & 14,600 & 20,500 & 0.325 & 3.635 & 0.207 \\
\hline 10,600 & 12,900 & 14,700 & 16,600 & 21,200 & 0.119 & 3.800 & 0.174 \\
\hline 2,830 & 3,480 & 3,970 & 4,470 & 5,640 & -0.113 & 3.194 & 0.203 \\
\hline 7,780 & 8,950 & 9,750 & 10,500 & 12,100 & -0.376 & 3.694 & 0.160 \\
\hline 3,720 & 4,830 & 5,780 & 6,840 & 9,810 & 0.548 & 3.319 & 0.190 \\
\hline 27,900 & 35,300 & 41,500 & 48,300 & 66,900 & 0.540 & 4.219 & 0.172 \\
\hline 44,300 & 57,300 & 68,200 & 80,100 & 113,000 & 0.394 & 4.386 & 0.198 \\
\hline 54,800 & 71,100 & 84,800 & 99,900 & 141,000 & 0.392 & 4.473 & 0.202 \\
\hline 167,000 & 198,000 & 220,000 & 243,000 & 295,000 & -0.056 & 5.020 & 0.160 \\
\hline 304,000 & 371,000 & 424,000 & 481,000 & 629,000 & 0.438 & 5.283 & 0.151 \\
\hline 4,560 & 6,120 & 7,450 & 8,930 & 13,100 & 0.300 & 3.349 & 0.236 \\
\hline 3,340 & 4,210 & 4,880 & 5,590 & 7,340 & 0.032 & 3.256 & 0.209 \\
\hline 8,540 & 9,860 & 10,800 & 11,700 & 13,600 & -0.272 & 3.738 & 0.155 \\
\hline 3,700 & 4,770 & 5,640 & 6,560 & 8,960 & 0.128 & 3.282 & 0.221 \\
\hline 2,100 & 2,750 & 3,300 & 3,920 & 5,630 & 0.439 & 3.053 & 0.204 \\
\hline 1,810 & 2,400 & 2,910 & 3,500 & 5,160 & 0.527 & 2.982 & 0.208 \\
\hline 878 & 1,060 & 1,210 & 1,360 & 1,750 & 0.414 & 2.752 & 0.145 \\
\hline 1,610 & 2,090 & 2,510 & 2,970 & 4,260 & 0.519 & 2.950 & 0.193 \\
\hline 4,750 & 5,960 & 6,910 & 7,900 & 10,400 & 0.087 & 3.418 & 0.201 \\
\hline 4,460 & 5,310 & 5,930 & 6,540 & 7,930 & -0.155 & 3.428 & 0.175 \\
\hline 9,010 & 10,900 & 12,500 & 14,100 & 18,100 & 0.328 & 3.752 & 0.154 \\
\hline 1,050 & 1,270 & 1,410 & 1,550 & 1,840 & -0.516 & 2.742 & 0.231 \\
\hline 10,600 & 12,200 & 13,400 & 14,500 & 17,000 & -0.222 & 3.833 & 0.152 \\
\hline 13,400 & -- & 17,700 & 19,500 & 23,800 & 0.025 & 3.931 & 0.153 \\
\hline 3,040 & 4,040 & 4,870 & 5,780 & 8,260 & 0.203 & 3.174 & 0.238 \\
\hline
\end{tabular}


Table 4. Selected flood-flow statistics for streamgage locations in and near Pennsylvania._Continued

$\left[\mathrm{ft}^{3} / \mathrm{s}\right.$; cubic feet per second; --, statistic not computed]

\begin{tabular}{|c|c|c|c|c|c|c|}
\hline $\begin{array}{c}\text { Streamgage } \\
\text { number }\end{array}$ & $\begin{array}{l}\text { Period of record used } \\
\text { in analysis }{ }^{1}\end{array}$ & $\begin{array}{l}\text { Number of } \\
\text { years of } \\
\text { systematic } \\
\text { record }\end{array}$ & $\begin{array}{l}\text { Number of } \\
\text { years used } \\
\text { in analysis }\end{array}$ & $\begin{array}{l}\text { Historical peak(s) } \\
\text { used in analysis }\end{array}$ & $\begin{array}{l}\text { 50-percent } \\
\text { annual } \\
\text { exceedance } \\
\left(\mathrm{ft}^{3} / \mathrm{s}\right)\end{array}$ & $\begin{array}{c}20 \text {-percent } \\
\text { annual } \\
\text { exceedance } \\
\left(\mathrm{ft}^{3} / \mathrm{s}\right)\end{array}$ \\
\hline 03099500 & $1944-2000$ & 48 & 48 & & 9,420 & 12,600 \\
\hline 03101500 & 1935-2008 & 75 & 75 & & 902 & 1,150 \\
\hline 03103500 & 1939-1991 & 52 & 52 & & 4,750 & 7,590 \\
\hline 03105500 & 1916-2008 & 79 & 79 & & 22,100 & 29,500 \\
\hline 03106300 & $1970-2008$ & 38 & 38 & & 346 & 477 \\
\hline 03106500 & 1969-2008 & 40 & 40 & & 6,290 & 7,970 \\
\hline 03107500 & 1936-2008 & 73 & 73 & & 33,300 & 44,300 \\
\hline 03111548 & 1982-2008 & 25 & 25 & & 2,660 & 4,160 \\
\hline 03112000 & ${ }^{2} 1987-2008$ & 22 & 22 & & 5,590 & 8,310 \\
\hline 03114000 & 1927-2003 & 53 & 53 & & 6,080 & 9,240 \\
\hline 04209000 & $1925-2008$ & 72 & 72 & & 8,900 & 13,400 \\
\hline 04212500 & 1924-1980 & 54 & 54 & & 4,470 & 6,460 \\
\hline
\end{tabular}

${ }^{1}$ Water year is defined as a 12-month period beginning October 1 and ending September 30. The water year is designated by the calendar year in which it ends.

${ }^{2}$ Period of record for post-regulation period.

${ }^{3}$ Period of record for pre-regulation period. 
Table 4. Selected flood-flow statistics for streamgage locations in and near Pennsylvania.-Continued [ft 3 /s; cubic feet per second; --, statistic not computed]

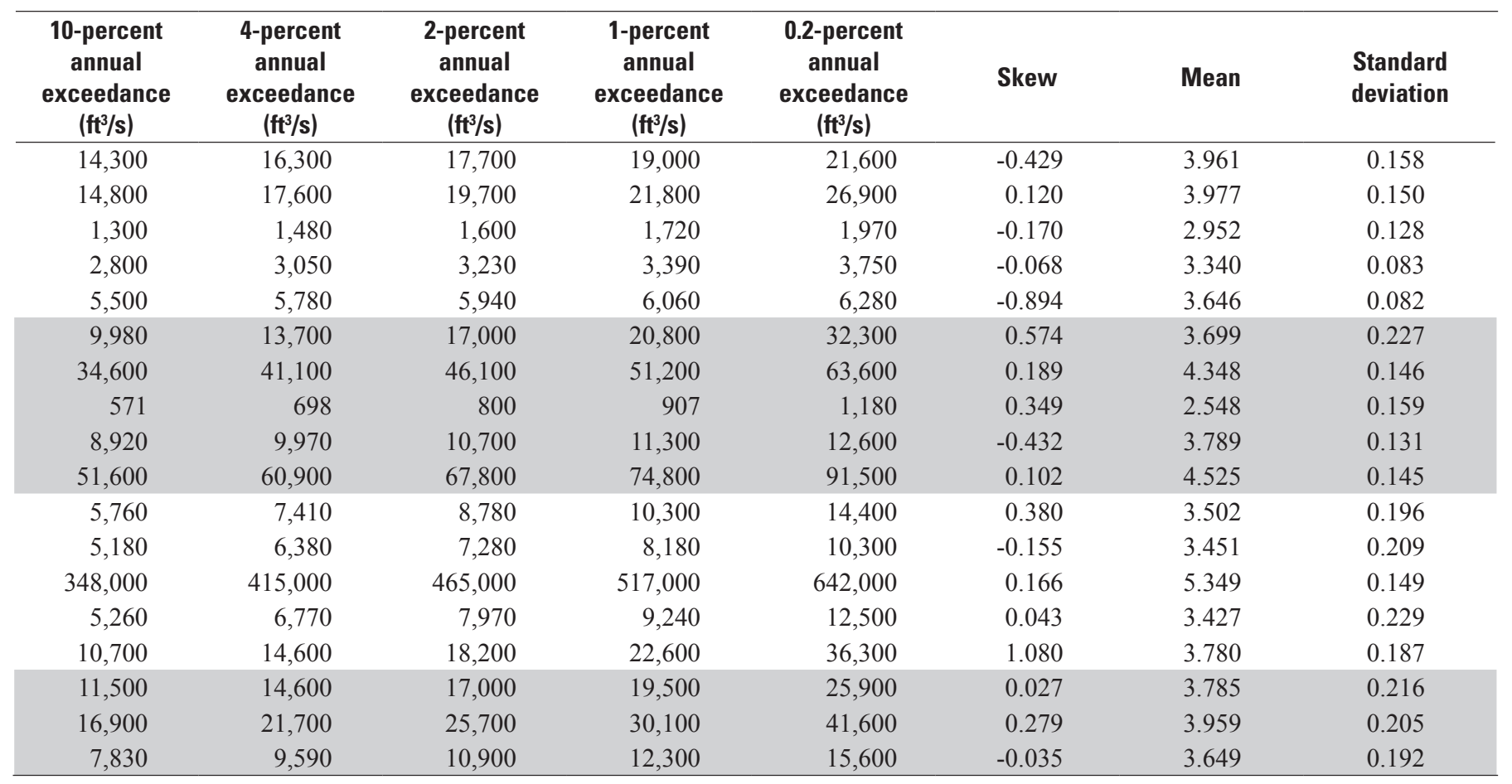


Table 5. Selected mean-flow and flow-duration statistics for streamgage locations in and near Pennsylvania.

$\left[\mathrm{ft}^{2} / \mathrm{s}\right.$; cubic feet per second; --, statistic not computed; <, less than]

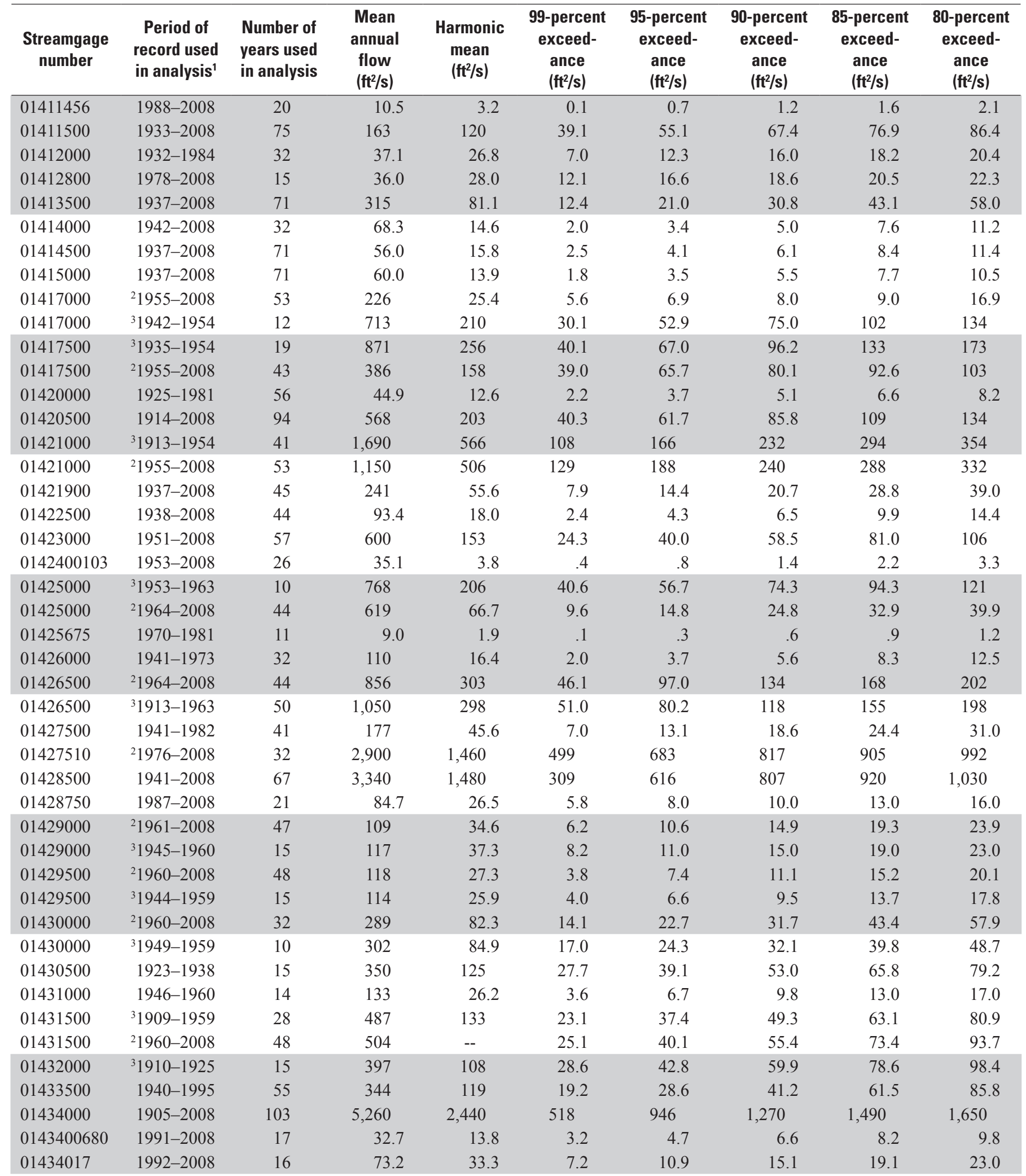


Table 5. Selected mean-flow and flow-duration statistics for streamgage locations in and near Pennsylvania.—Continued [ $\mathrm{ft} 3 / \mathrm{s}$; cubic feet per second; --, statistic not computed; $<$, less than]

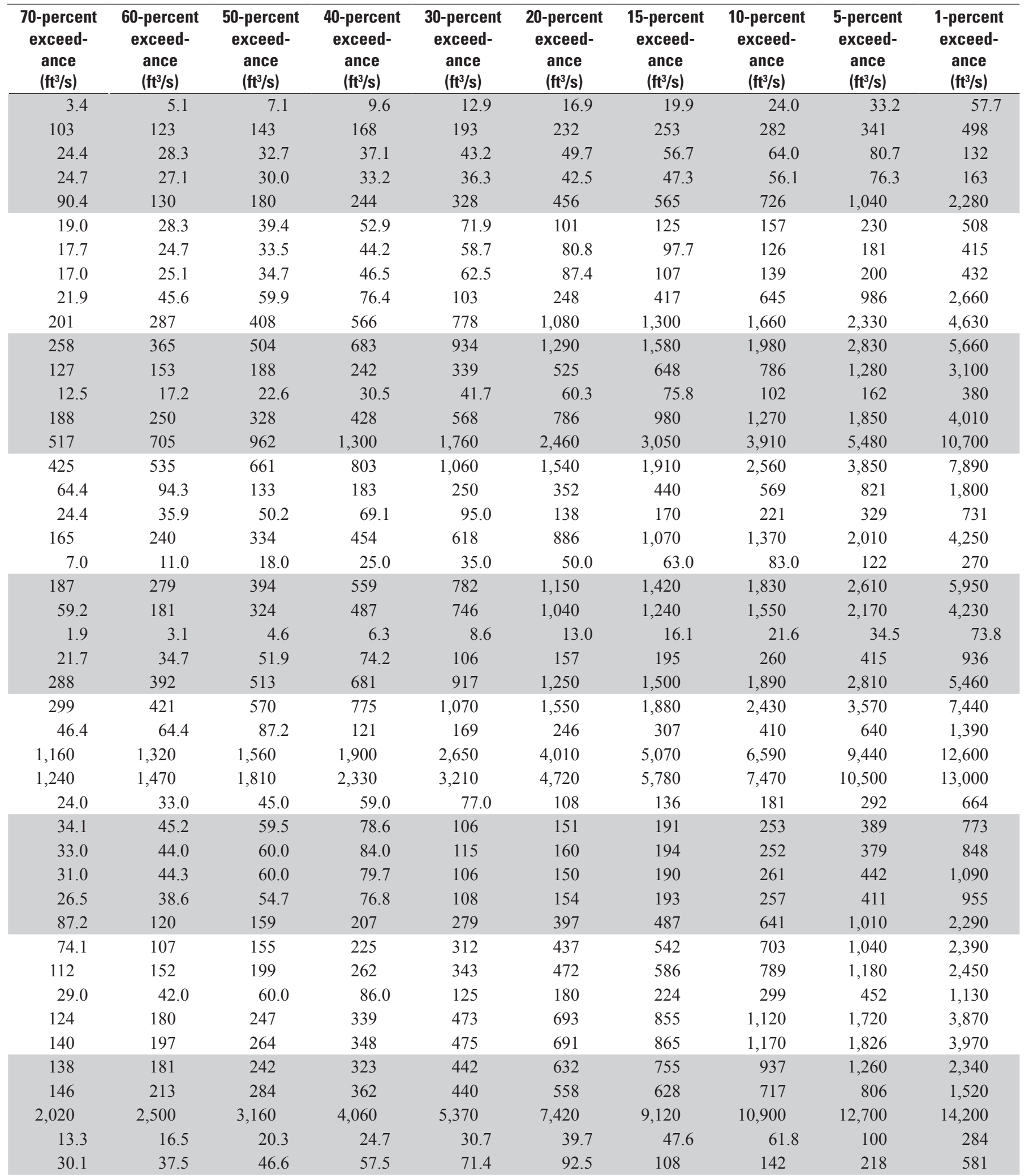


Table 5. Selected mean-flow and flow-duration statistics for streamgage locations in and near Pennsylvania.—Continued

[ft²/s; cubic feet per second; --, statistic not computed; <, less than]

\begin{tabular}{|c|c|c|c|c|c|c|c|c|c|}
\hline $\begin{array}{c}\text { Streamgage } \\
\text { number }\end{array}$ & $\begin{array}{l}\text { Period of } \\
\text { record used } \\
\text { in analysis }{ }^{1}\end{array}$ & $\begin{array}{l}\text { Number of } \\
\text { years used } \\
\text { in analysis }\end{array}$ & $\begin{array}{l}\text { Mean } \\
\text { annual } \\
\text { flow } \\
\left(\mathrm{ft}^{2} / \mathrm{s}\right)\end{array}$ & $\begin{array}{c}\text { Harmonic } \\
\text { mean } \\
\left(\mathrm{ft}^{2} / \mathrm{s}\right)\end{array}$ & $\begin{array}{l}\text { 99-percent } \\
\text { exceed- } \\
\text { ance } \\
\left(\mathrm{ft}^{2} / \mathrm{s}\right)\end{array}$ & $\begin{array}{l}\text { 95-percent } \\
\text { exceed- } \\
\text { ance } \\
\left(\mathrm{ft}^{2} / \mathrm{s}\right)\end{array}$ & $\begin{array}{l}\text { 90-percent } \\
\text { exceed- } \\
\text { ance } \\
\left(\mathrm{ft}^{2} / \mathrm{s}\right)\end{array}$ & $\begin{array}{l}\text { 85-percent } \\
\text { exceed- } \\
\text { ance } \\
\left(\mathrm{ft}^{2} / \mathrm{s}\right)\end{array}$ & $\begin{array}{l}\text { 80-percent } \\
\text { exceed- } \\
\text { ance } \\
\left(\mathrm{ft}^{2} / \mathrm{s}\right)\end{array}$ \\
\hline 01434025 & 1984-2008 & 24 & 10.7 & 3.5 & .2 & .9 & 1.6 & 2.2 & 2.7 \\
\hline 01434498 & 1992-2008 & 16 & 111 & 45.8 & 8.5 & 13.4 & 19.6 & 26.3 & 32.2 \\
\hline 01436000 & ${ }^{3} 1942-1953$ & 11 & 263 & 112 & 19.1 & 33.0 & 45.2 & 57.4 & 69.0 \\
\hline 01436500 & ${ }^{2} 1954-1993$ & 35 & 89.9 & 46.2 & 16.1 & 19.7 & 23.7 & 27.4 & 31.4 \\
\hline 01436500 & ${ }^{3} 1938-1953$ & 15 & 300 & 122 & 25.2 & 37.5 & 51.0 & 64.7 & 78.6 \\
\hline 01436690 & 1993-2008 & 15 & 246 & 127 & 38.3 & 57.1 & 70.7 & 80.8 & 90.6 \\
\hline 01437500 & ${ }^{2} 1954-2008$ & 54 & 442 & 224 & 63.2 & 88.5 & 110 & 130 & 149 \\
\hline 01440000 & 1924-2008 & 84 & 112 & 41.9 & 8.2 & 13.2 & 17.7 & 21.9 & 27.0 \\
\hline 01440200 & 1965-1995 & 30 & 6,210 & 3,490 & 1,090 & 1,530 & 1,800 & 2,020 & 2,200 \\
\hline 01440400 & 1958-2008 & 50 & 140 & 45.5 & 7.6 & 12.0 & 17.0 & 23.0 & 30.0 \\
\hline 01441000 & 1912-1938 & 26 & 121 & 59.1 & 16.5 & 23.4 & 28.7 & 34.3 & 40.0 \\
\hline 01442500 & 1951-2008 & 57 & 573 & 228 & 50.0 & 73.0 & 95.0 & 119 & 145 \\
\hline 01443280 & 1993-2008 & 15 & 22.5 & 12.5 & 1.9 & 5.5 & 7.2 & 8.3 & 9.4 \\
\hline 01443500 & $1922-2008$ & 85 & 201 & 83.1 & 17.7 & 26.9 & 35.3 & 43.9 & 53.3 \\
\hline 01443900 & 1967-2008 & 41 & 10.9 & 2.9 & .2 & 1.0 & 1.3 & 1.6 & 1.8 \\
\hline 01445000 & 1940-2008 & 28 & 49.6 & 17.4 & 2.5 & 4.5 & 6.6 & 9.3 & 12.5 \\
\hline 01445500 & $1922-2008$ & 86 & 160 & 79.8 & 19.2 & 28.9 & 36.2 & 44.1 & 52.4 \\
\hline 01446000 & 1923-2008 & 43 & 54.9 & 17.0 & 2.4 & 4.5 & 6.6 & 9.3 & 12.5 \\
\hline 01447800 & 1958-2008 & 50 & 640 & 264 & 51.1 & 80.3 & 115 & 149 & 182 \\
\hline 01448000 & 1917-1959 & 42 & 669 & 308 & 73.6 & 106 & 139 & 169 & 198 \\
\hline 01448500 & 1949-1996 & 47 & 4.9 & 2.0 & .4 & .7 & .9 & 1.2 & 1.4 \\
\hline 01449000 & 1983-2008 & 25 & 1,340 & 664 & 160 & 231 & 300 & 364 & 435 \\
\hline 01449360 & 1967-2008 & 41 & 104 & 57.6 & 15.0 & 22.0 & 28.0 & 32.0 & 38.0 \\
\hline 01449800 & 1968-2008 & 40 & 170 & 75.0 & 16.1 & 30.0 & 36.7 & 42.6 & 48.3 \\
\hline 01450500 & 1940-2008 & 68 & 153 & 74.4 & 18.0 & 27.0 & 35.0 & 42.0 & 49.0 \\
\hline 01451000 & ${ }^{2} 1961-2008$ & 47 & 1,880 & 916 & 212 & 324 & 413 & 505 & 606 \\
\hline 01451000 & ${ }^{3} 1947-1960$ & 13 & 1,940 & 936 & 225 & 329 & 425 & 521 & 622 \\
\hline 01451500 & 1946-2008 & 62 & 104 & 72.0 & 28.0 & 35.0 & 41.0 & 45.0 & 50.0 \\
\hline 01451650 & 1987-2008 & 21 & 138 & 96.2 & 39.0 & 48.2 & 55.3 & 61.5 & 67.8 \\
\hline 01451800 & 1966-2008 & 42 & 94.2 & 23.4 & 3.5 & 7.2 & 11.0 & 15.0 & 19.0 \\
\hline 01452000 & 1945-2008 & 63 & 118 & 28.2 & 3.7 & 7.7 & 12.0 & 17.0 & 22.0 \\
\hline 01452500 & 1949-2008 & 59 & 55.3 & 36.7 & 11.0 & 17.0 & 21.0 & 24.0 & 26.0 \\
\hline 01453000 & ${ }^{2} 1928-2008$ & 47 & 2,510 & 1,410 & 374 & 556 & 699 & 822 & 950 \\
\hline
\end{tabular}


Table 5. Selected mean-flow and flow-duration statistics for streamgage locations in and near Pennsylvania.-Continued [ $\mathrm{ft}^{3} / \mathrm{s}$; cubic feet per second; --, statistic not computed; <, less than]

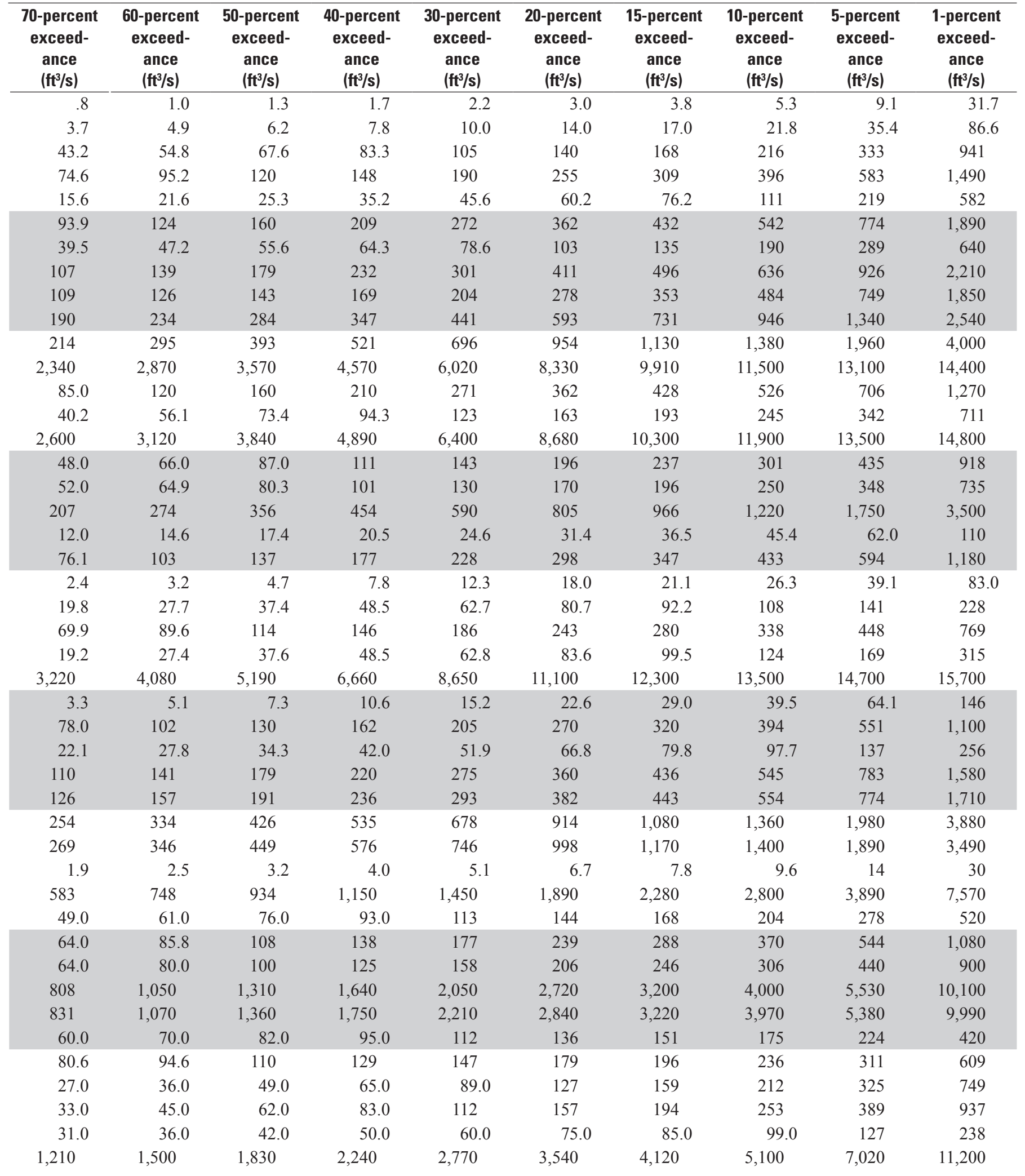


Table 5. Selected mean-flow and flow-duration statistics for streamgage locations in and near Pennsylvania.-Continued

[ft²/s; cubic feet per second; --, statistic not computed; <, less than]

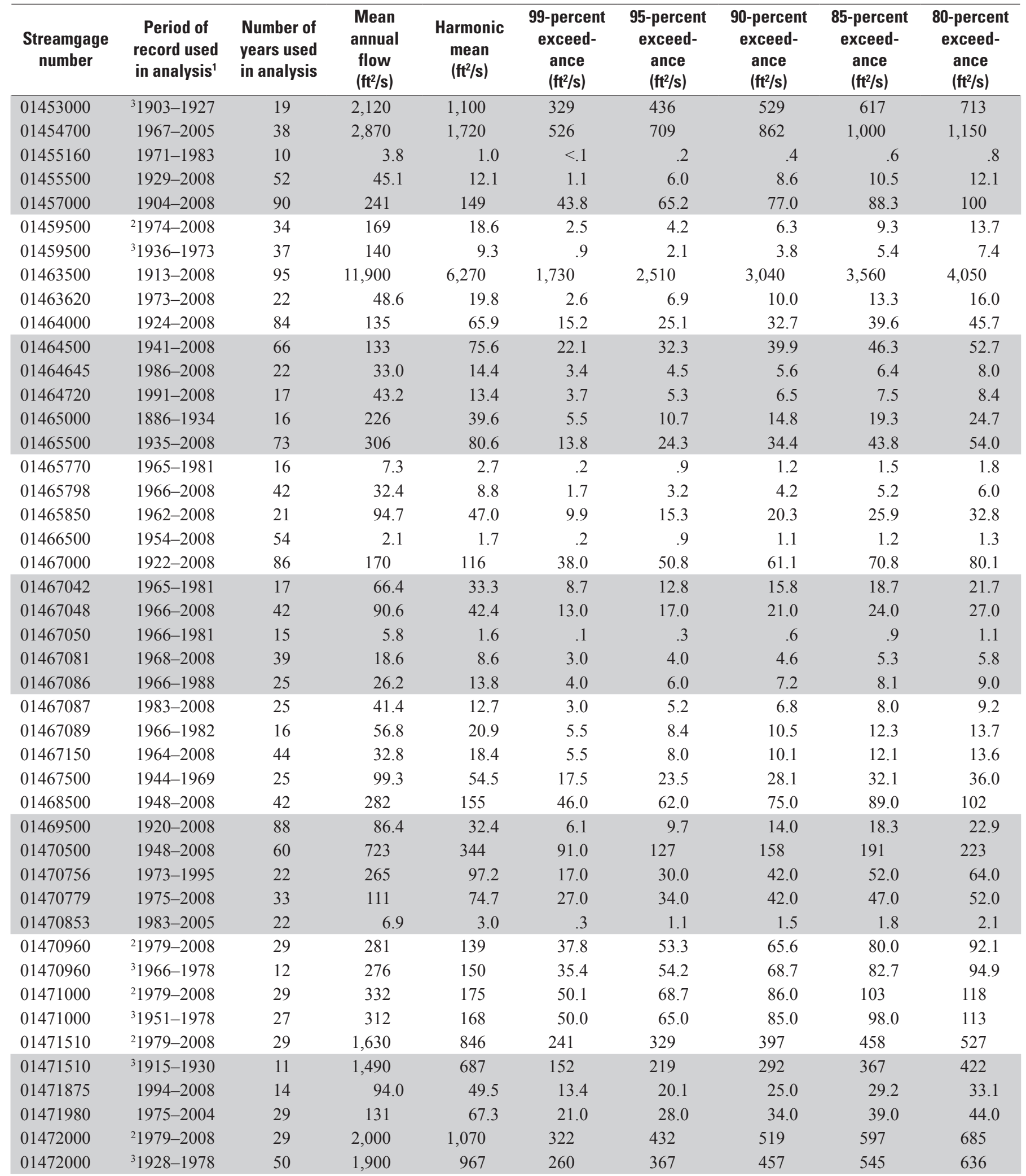


Table 5. Selected mean-flow and flow-duration statistics for streamgage locations in and near Pennsylvania.-Continued [ $\mathrm{ft}^{3} / \mathrm{s}$; cubic feet per second; --, statistic not computed; $<$, less than]

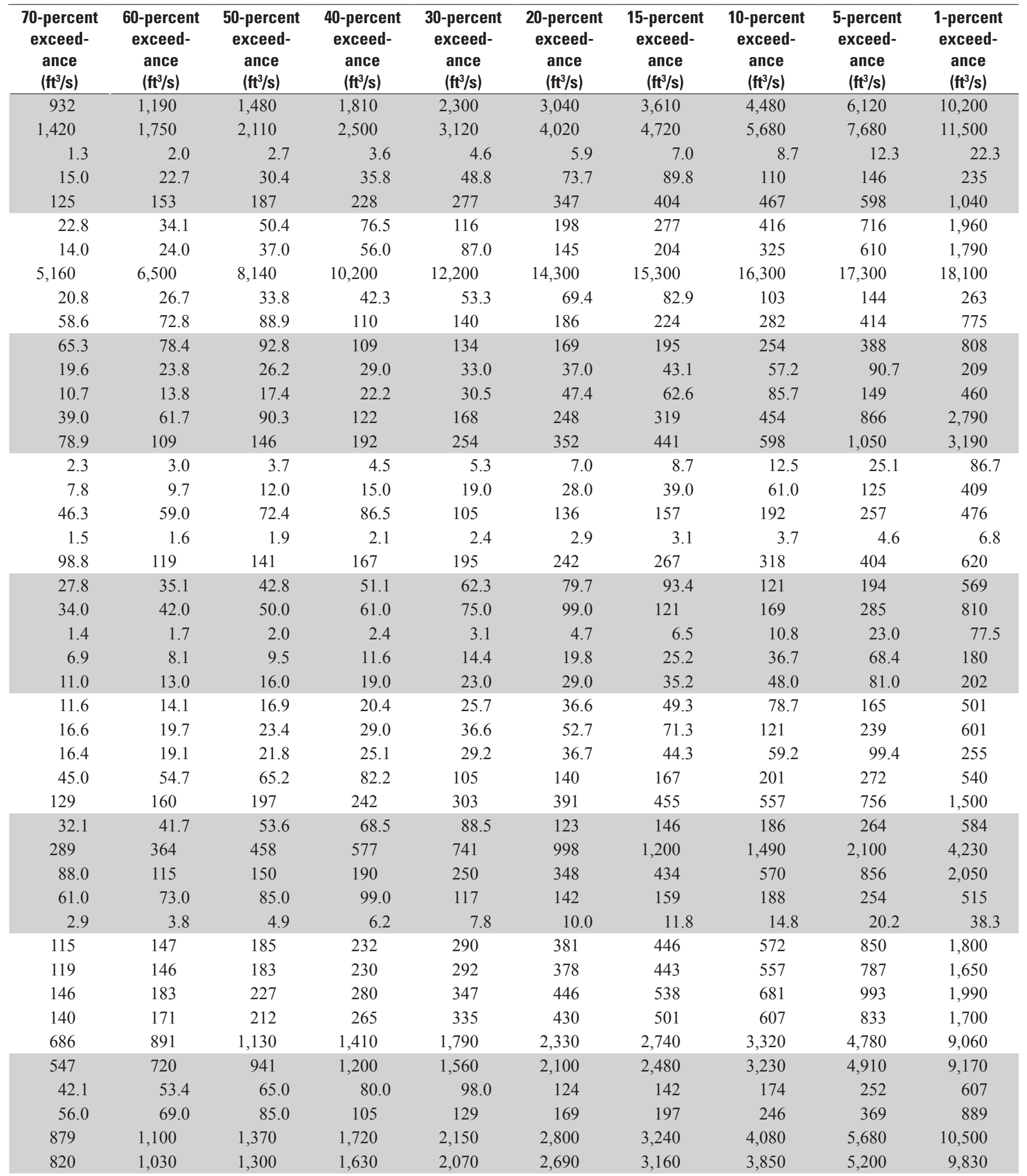


Table 5. Selected mean-flow and flow-duration statistics for streamgage locations in and near Pennsylvania.-Continued

[ft²/s; cubic feet per second; --, statistic not computed; <, less than]

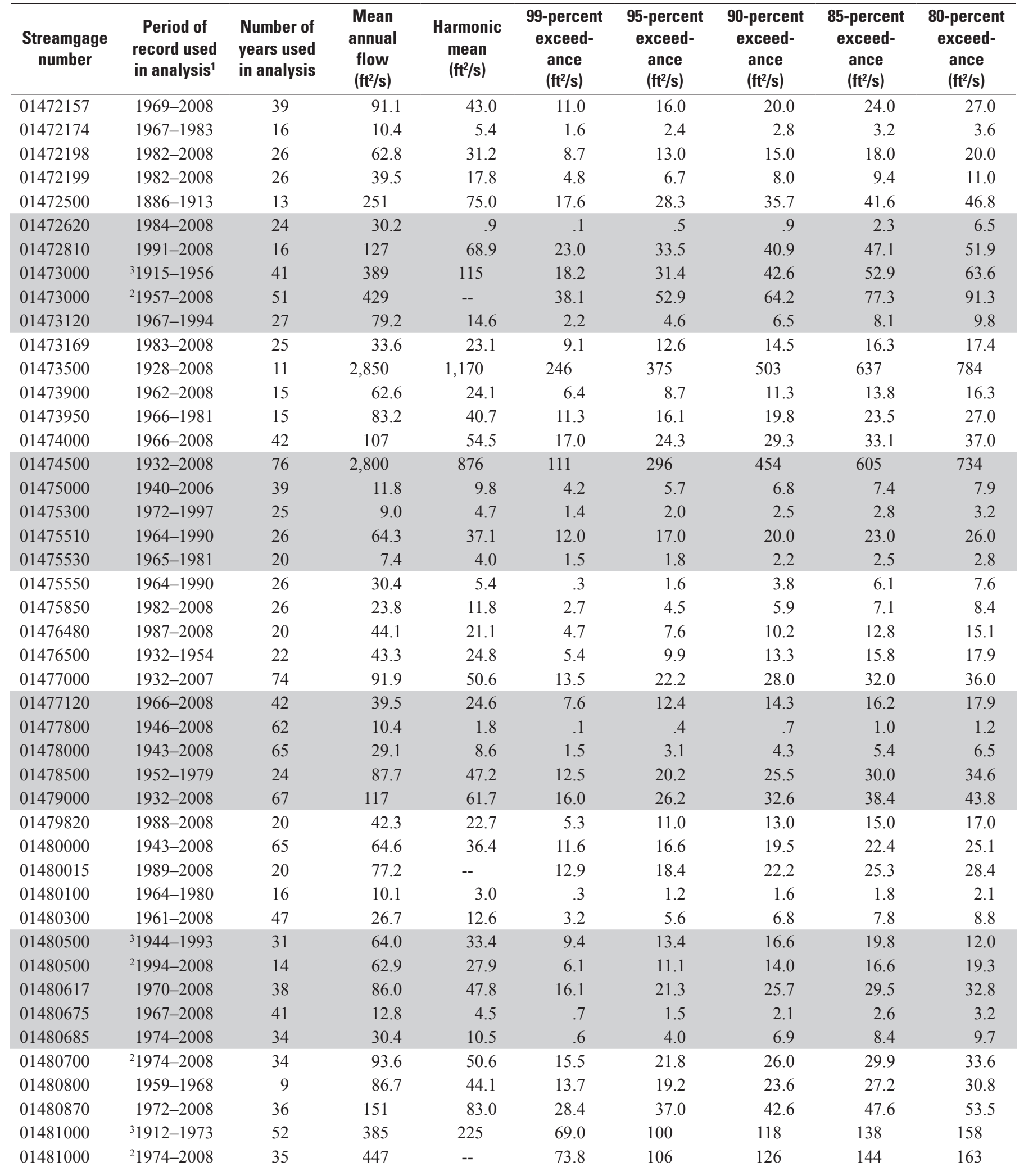


Table 5. Selected mean-flow and flow-duration statistics for streamgage locations in and near Pennsylvania.—Continued $\left[\mathrm{ft}^{3} / \mathrm{s}\right.$; cubic feet per second; --, statistic not computed; <, less than]

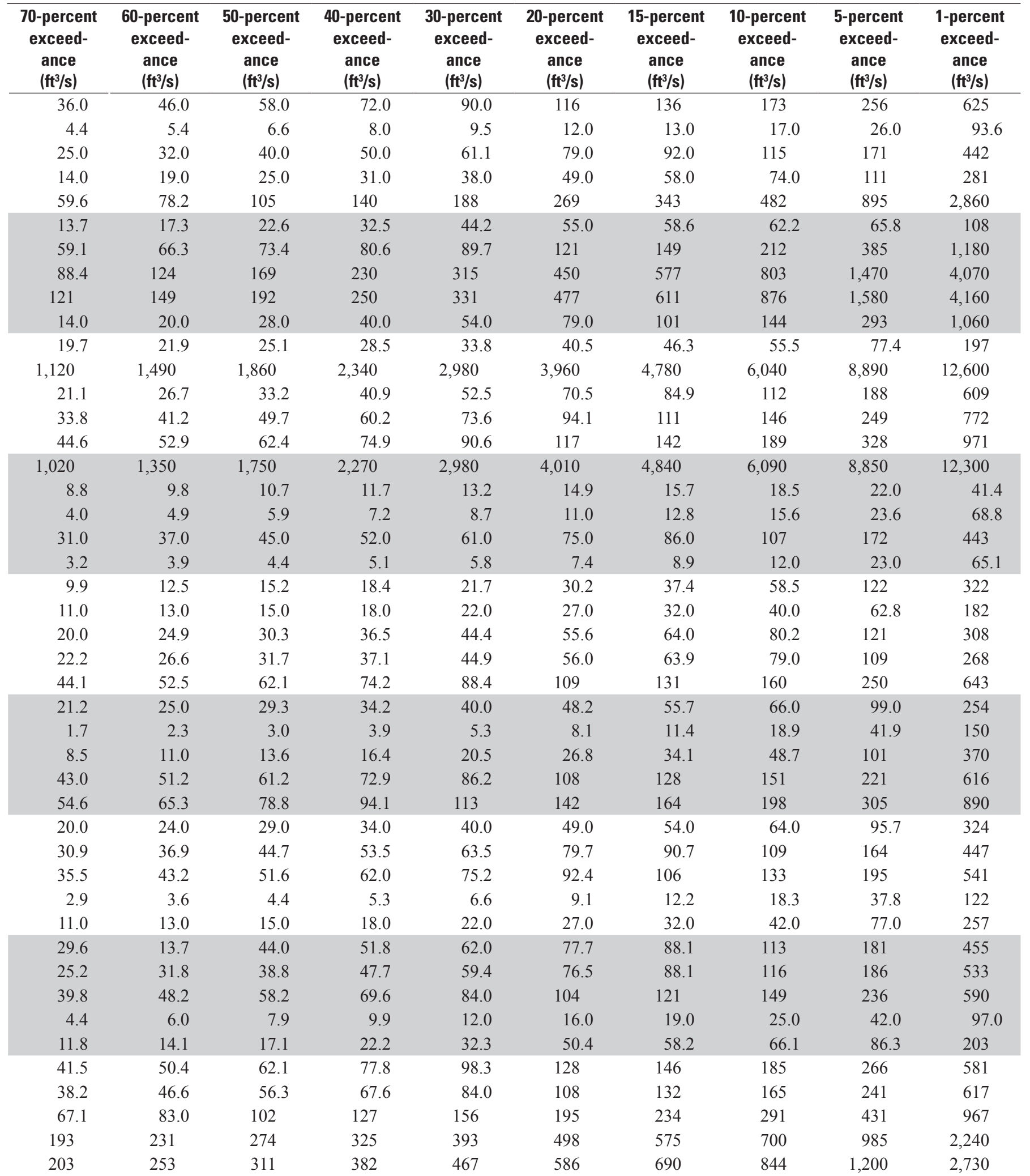


Table 5. Selected mean-flow and flow-duration statistics for streamgage locations in and near Pennsylvania._-Continued [ $\mathrm{ft}^{2} / \mathrm{s}$; cubic feet per second; --, statistic not computed; $<$, less than]

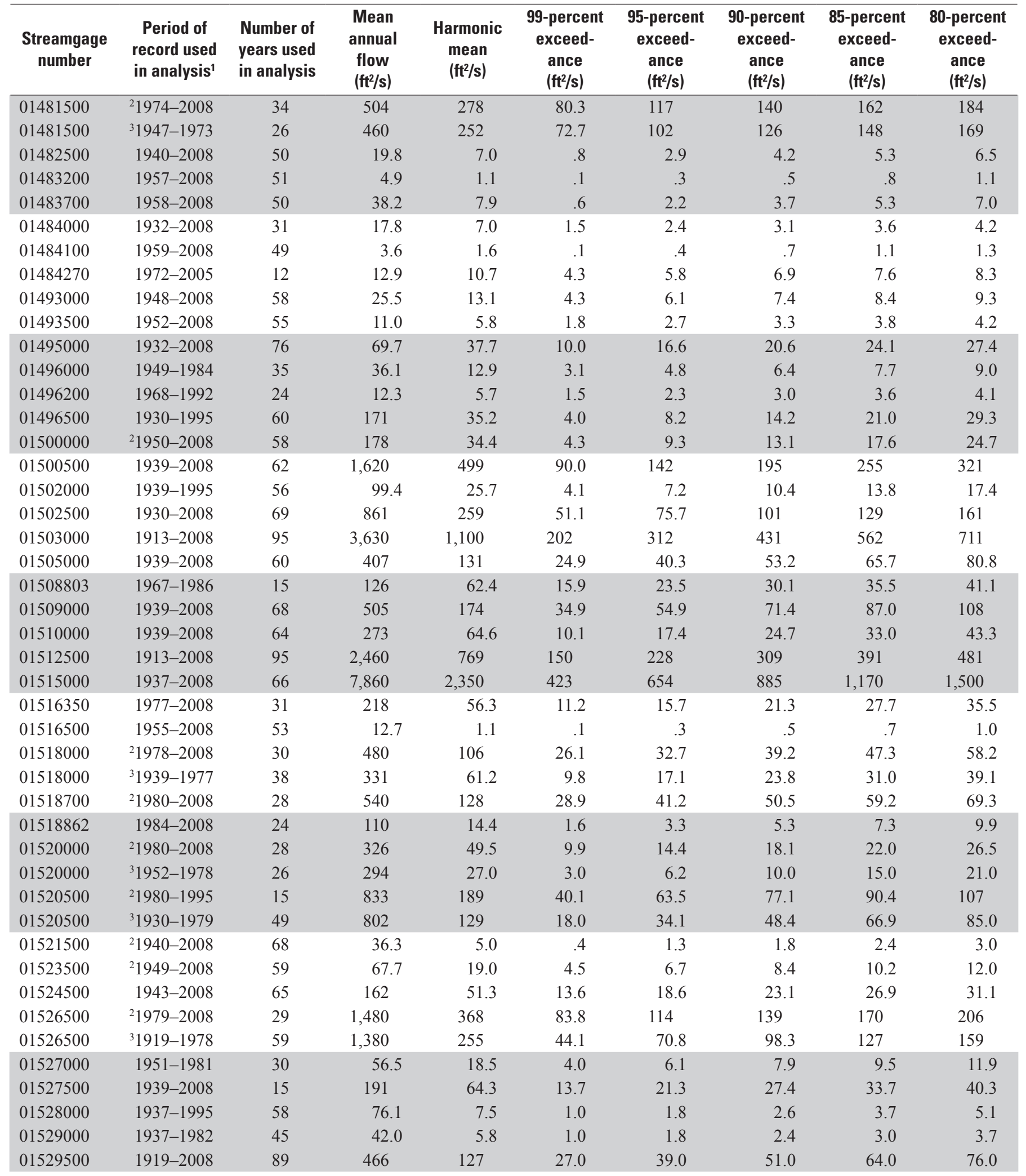


Table 5. Selected mean-flow and flow-duration statistics for streamgage locations in and near Pennsylvania.—Continued $[\mathrm{ft} / \mathrm{s}$; cubic feet per second; --, statistic not computed; <, less than]

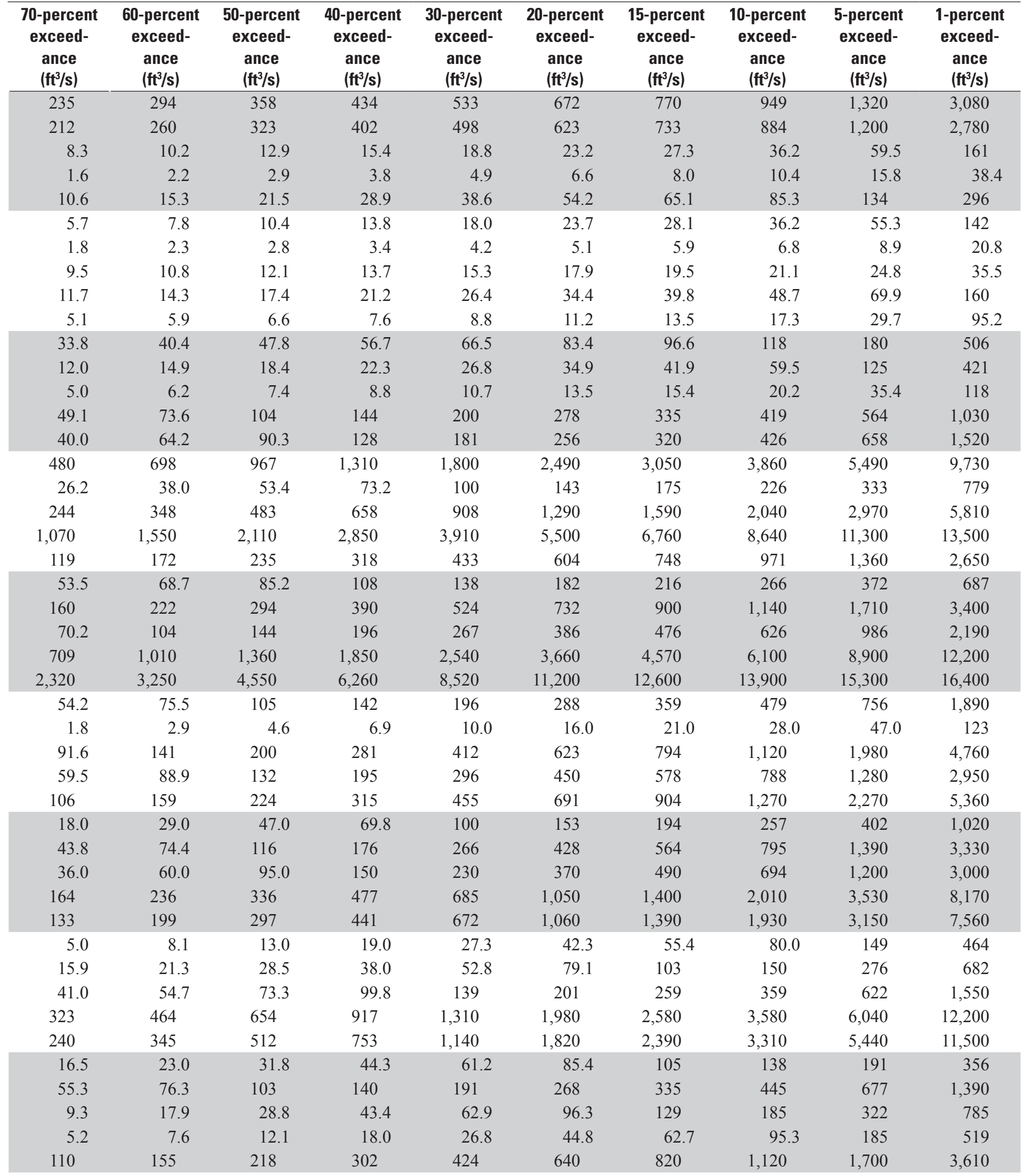


Table 5. Selected mean-flow and flow-duration statistics for streamgage locations in and near Pennsylvania.-Continued

$\left[\mathrm{ft}^{2} / \mathrm{s}\right.$; cubic feet per second; --, statistic not computed; $<$, less than]

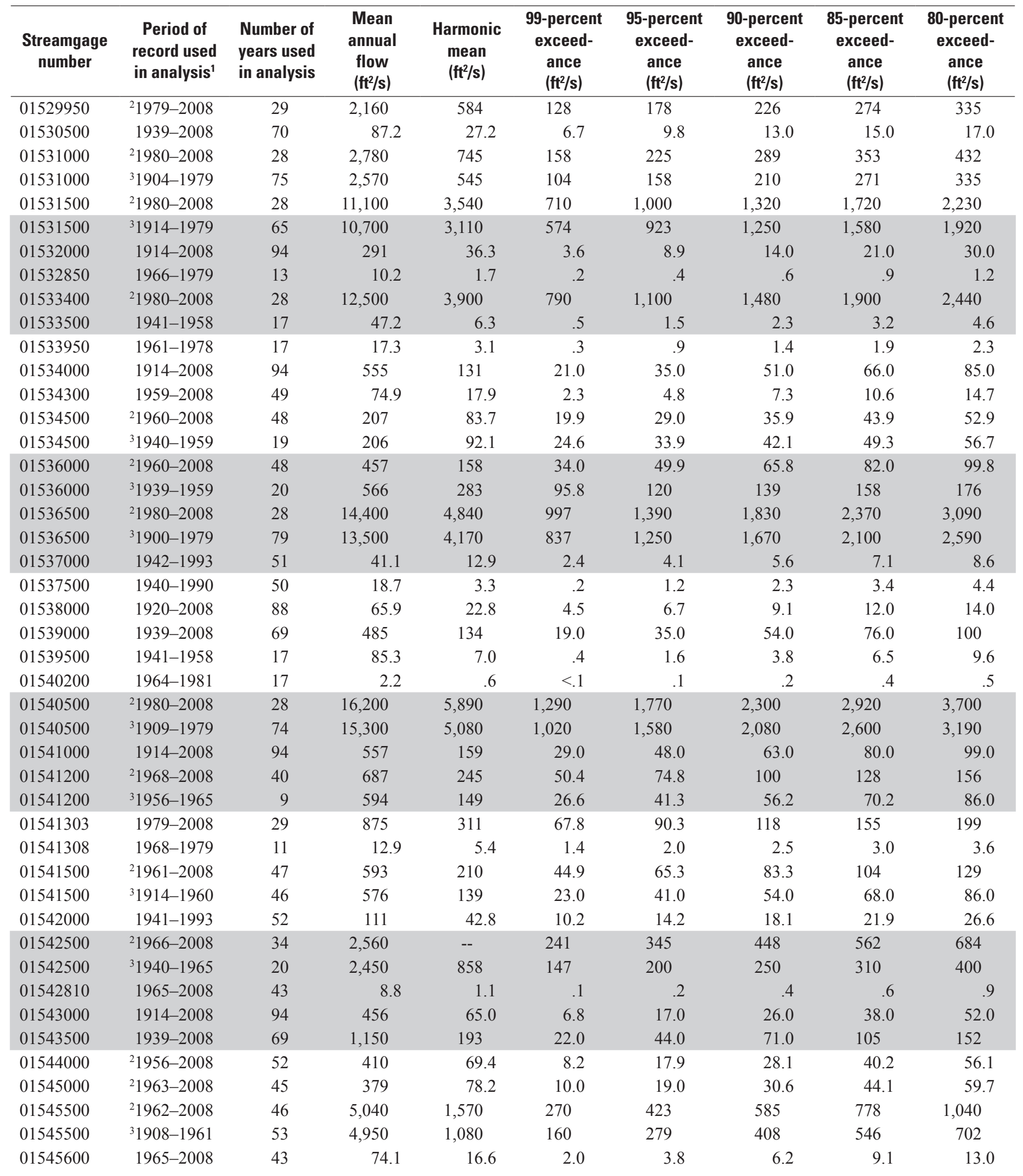


Table 5. Selected mean-flow and flow-duration statistics for streamgage locations in and near Pennsylvania.—Continued $\left[\mathrm{ft}^{3} / \mathrm{s}\right.$; cubic feet per second; --, statistic not computed; <, less than]

\begin{tabular}{|c|c|c|c|c|c|c|c|c|c|}
\hline $\begin{array}{c}\text { 70-percent } \\
\text { exceed- } \\
\text { ance } \\
\left(\mathrm{ft}^{3} / \mathrm{s}\right)\end{array}$ & $\begin{array}{c}\text { 60-percent } \\
\text { exceed- } \\
\text { ance } \\
\left(\mathrm{ft}^{3} / \mathrm{s}\right)\end{array}$ & $\begin{array}{c}\text { 50-percent } \\
\text { exceed- } \\
\text { ance } \\
\left(\mathrm{ft}^{3} / \mathrm{s}\right)\end{array}$ & $\begin{array}{c}\text { 40-percent } \\
\text { exceed- } \\
\text { ance } \\
\left(\mathrm{ft}^{3} / \mathrm{s}\right)\end{array}$ & $\begin{array}{c}\text { 30-percent } \\
\text { exceed- } \\
\text { ance } \\
\left(\mathrm{ft}^{3} / \mathrm{s}\right)\end{array}$ & $\begin{array}{c}\text { 20-percent } \\
\text { exceed- } \\
\text { ance } \\
\left(\mathrm{ft}^{3} / \mathrm{s}\right)\end{array}$ & $\begin{array}{c}\text { 15-percent } \\
\text { exceed- } \\
\text { ance } \\
\left(\mathrm{ft}^{3} / \mathrm{s}\right)\end{array}$ & $\begin{array}{c}\text { 10-percent } \\
\text { exceed- } \\
\text { ance } \\
\left(\mathrm{ft}^{3} / \mathrm{s}\right)\end{array}$ & $\begin{array}{c}\text { 5-percent } \\
\text { exceed- } \\
\text { ance } \\
\left(\mathrm{ft}^{3} / \mathrm{s}\right)\end{array}$ & $\begin{array}{c}\text { 1-percent } \\
\text { exceed- } \\
\text { ance } \\
\left(\mathrm{ft}^{3} / \mathrm{s}\right)\end{array}$ \\
\hline 23.0 & 29.0 & 39.0 & 51.0 & 71.0 & 106 & 135 & 188 & 307 & 792 \\
\hline 671 & 959 & 1,330 & 1,840 & 2,520 & 3,800 & 4,840 & 6,490 & 10,300 & 14,300 \\
\hline 495 & 706 & 1,010 & 1,470 & 2,190 & 3,410 & 4,420 & 6,230 & 9,870 & 13,700 \\
\hline 2,820 & 3,940 & 5,460 & 7,560 & 10,600 & 13,700 & 15,200 & 16,700 & 18,300 & 19,500 \\
\hline 52.0 & 80.0 & 120 & 170 & 246 & 377 & 482 & 662 & 1,080 & 2,670 \\
\hline 2.0 & 3.0 & 4.2 & 6.0 & 8.0 & 12.0 & 15.0 & 21.0 & 34.0 & 107 \\
\hline 3,790 & 5,280 & 7,140 & 9,520 & 12,000 & 14,500 & 15,700 & 17,000 & 18,200 & 19,200 \\
\hline 8.3 & 13.9 & 20.5 & 30.1 & 44.9 & 66.0 & 83.0 & 109 & 174 & 376 \\
\hline 73.3 & 94.9 & 124 & 162 & 215 & 292 & 347 & 441 & 653 & 1,390 \\
\hline 140 & 190 & 256 & 344 & 461 & 674 & 838 & 1,090 & 1,580 & 2,940 \\
\hline 217 & 268 & 344 & 453 & 594 & 804 & 985 & 1,230 & 1,730 & 3,260 \\
\hline 4,630 & 6,400 & 8,530 & 11,100 & 13,800 & 16,400 & 17,700 & 19,000 & 20,300 & 21,300 \\
\hline 3,770 & 5,200 & 7,050 & 9,750 & 12,700 & 15,600 & 17,100 & 18,600 & 20,100 & 21,300 \\
\hline 12.3 & 15.8 & 20.9 & 27.9 & 39.2 & 58.1 & 72.4 & 95.5 & 146 & 309 \\
\hline 6.3 & 8.3 & 11.3 & 15.0 & 20.0 & 27.2 & 33.1 & 40.6 & 57.0 & 126 \\
\hline 22.0 & 31.0 & 41.0 & 53.0 & 70.0 & 94.0 & 112 & 140 & 200 & 422 \\
\hline 150 & 208 & 278 & 369 & 498 & 692 & 846 & 1,080 & 1,570 & 3,210 \\
\hline 16.7 & 25.5 & 37.6 & 55.5 & 80.4 & 122 & 150 & 204 & 324 & 787 \\
\hline 289 & 397 & 528 & 707 & 951 & 1,310 & 1,620 & 2,080 & 3,130 & 5,180 \\
\hline 4.8 & 6.2 & 7.7 & 9.8 & 13.0 & 17.0 & 21.0 & 26.0 & 40.0 & 89.0 \\
\hline 186 & 255 & 340 & 447 & 607 & 859 & 1,060 & 1,360 & 2,000 & 4,010 \\
\hline 130 & 190 & 278 & 398 & 575 & 850 & 1,060 & 1,380 & 2,010 & 4,350 \\
\hline 37.6 & 51.0 & 67.9 & 90.5 & 122 & 163 & 194 & 246 & 346 & 712 \\
\hline 955 & 1,260 & 1,650 & 2,130 & 2,810 & 3,840 & 4,600 & 5,760 & 8,040 & 11,800 \\
\hline 620 & 916 & 1,260 & 1,800 & 2,590 & 3,740 & 4,560 & 5,770 & 8,100 & 15,000 \\
\hline 1.5 & 2.4 & 3.6 & 5.2 & 7.5 & 11.0 & 15.0 & 21.0 & 35.0 & 84.0 \\
\hline 92.0 & 142 & 210 & 294 & 430 & 652 & 815 & 1,100 & 1,680 & 3,660 \\
\hline 262 & 404 & 580 & 808 & 1,160 & 1,720 & 2,140 & 2,780 & 4,100 & 8,200 \\
\hline 94.9 & 141 & 201 & 283 & 401 & 590 & 748 & 1,010 & 1,570 & 3,290 \\
\hline 95.0 & 139 & 193 & 263 & 361 & 538 & 674 & 908 & 1,360 & 3,180 \\
\hline 1,620 & 2,290 & 3,080 & 4,100 & 5,480 & 7,500 & 9,200 & 11,000 & 12,800 & 14,200 \\
\hline 1,160 & 1,790 & 2,560 & 3,620 & 5,170 & 7,500 & 9,380 & 11,300 & 13,300 & 14,900 \\
\hline 21.0 & 31.0 & 42.0 & 56.0 & 77.0 & 109 & 133 & 170 & 248 & 489 \\
\hline
\end{tabular}


Table 5. Selected mean-flow and flow-duration statistics for streamgage locations in and near Pennsylvania.—Continued $\left[\mathrm{ft}^{2} / \mathrm{s}\right.$; cubic feet per second; --, statistic not computed; <, less than]

\begin{tabular}{|c|c|c|c|c|c|c|c|c|c|}
\hline $\begin{array}{c}\text { Streamgage } \\
\text { number }\end{array}$ & $\begin{array}{l}\text { Period of } \\
\text { record used } \\
\text { in analysis }{ }^{1}\end{array}$ & $\begin{array}{l}\text { Number of } \\
\text { years used } \\
\text { in analysis }\end{array}$ & $\begin{array}{c}\text { Mean } \\
\text { annual } \\
\text { flow } \\
\left(\mathrm{ft}^{2} / \mathrm{s}\right)\end{array}$ & $\begin{array}{c}\text { Harmonic } \\
\text { mean } \\
\left(\mathrm{ft}^{2} / \mathrm{s}\right)\end{array}$ & $\begin{array}{l}\text { 99-percent } \\
\text { exceed- } \\
\text { ance } \\
\left(\mathrm{ft}^{2} / \mathrm{s}\right)\end{array}$ & $\begin{array}{c}\text { 95-percent } \\
\text { exceed- } \\
\text { ance } \\
\left(\mathrm{ft}^{2} / \mathrm{s}\right)\end{array}$ & $\begin{array}{l}\text { 90-percent } \\
\text { exceed- } \\
\text { ance } \\
\left(\mathrm{ft}^{2} / \mathrm{s}\right)\end{array}$ & $\begin{array}{l}\text { 85-percent } \\
\text { exceed- } \\
\text { ance } \\
\left(\mathrm{ft}^{2} / \mathrm{s}\right)\end{array}$ & $\begin{array}{c}\text { 80-percent } \\
\text { exceed- } \\
\text { ance } \\
\left(\mathrm{ft}^{2} / \mathrm{s}\right)\end{array}$ \\
\hline 01546400 & 1985-2008 & 23 & 69.1 & 42.3 & 16.0 & 19.0 & 22.0 & 25.0 & 28.0 \\
\hline 01546500 & $1941-2008$ & 67 & 95.6 & 68.3 & 25.0 & 35.0 & 41.0 & 45.0 & 48.0 \\
\hline 01547500 & ${ }^{2} 1970-2008$ & 38 & 507 & 288 & 114 & 138 & 159 & 174 & 188 \\
\hline 01547500 & ${ }^{3} 1955-1969$ & 14 & 389 & 220 & 91.6 & 110 & 121 & 133 & 144 \\
\hline 01547700 & 1956-2008 & 52 & 60.2 & 8.1 & .8 & 2.1 & 3.4 & 4.8 & 6.6 \\
\hline 01547800 & 1970-1981 & 11 & 23.6 & 9.1 & 2.0 & 3.0 & 3.8 & 4.8 & 5.9 \\
\hline 01547950 & 1969-2008 & 39 & 266 & 88.6 & 15.0 & 23.0 & 34.0 & 47.0 & 60.0 \\
\hline 01549000 & 1909-1920 & 11 & 1,140 & 264 & 42.9 & 67.4 & 99.2 & 134 & 176 \\
\hline 01549500 & 1941-2008 & 67 & 58.8 & 9.8 & 1.1 & 2.4 & 3.7 & 5.5 & 7.7 \\
\hline 01549700 & 1958-2008 & 50 & 1,410 & 309 & 43.0 & 75.0 & 112 & 156 & 205 \\
\hline 01550000 & 1914-2008 & 94 & 290 & 66.1 & 9.5 & 17.0 & 25.0 & 35.0 & 46.0 \\
\hline 01551500 & ${ }^{2} 1962-2008$ & 46 & 9,240 & 3,230 & 624 & 913 & 1,250 & 1,660 & 2,100 \\
\hline 01551500 & ${ }^{3} 1900-1961$ & 61 & 8,920 & 2,520 & 485 & 724 & 960 & 1,230 & 1,570 \\
\hline 01552000 & 1926-2008 & 81 & 767 & 184 & 26.0 & 44.6 & 70.0 & 103 & 137 \\
\hline 01552500 & 1941-2008 & 67 & 48.6 & 11.1 & 1.5 & 2.7 & 4.4 & 6.4 & 8.8 \\
\hline 01553130 & 1968-1981 & 13 & 9.1 & 4.0 & 1.1 & 1.6 & 2.0 & 2.2 & 2.5 \\
\hline 01553500 & ${ }^{3} 1940-1966$ & 27 & 10,200 & -- & 647 & 909 & 1,170 & 1,440 & 1,790 \\
\hline 01556000 & $1917-2008$ & 91 & 400 & 159 & 48.0 & 63.0 & 73.0 & 81.0 & 93.0 \\
\hline 01557500 & 1945-2008 & 63 & 76.0 & 20.5 & 3.6 & 5.2 & 7.0 & 9.0 & 12.0 \\
\hline 01558000 & 1939-2008 & 69 & 379 & 178 & 64.0 & 74.0 & 83.0 & 92.0 & 103 \\
\hline 01559000 & $1942-2008$ & 66 & 1,110 & 540 & 173 & 228 & 266 & 297 & 330 \\
\hline 01559500 & $1930-1958$ & 28 & 148 & 44.5 & 12.1 & 15.7 & 18.7 & 21.7 & 25.8 \\
\hline 01559700 & $1962-1978$ & 16 & 5.5 & .7 & .1 & .2 & .3 & .4 & .4 \\
\hline 01560000 & 1940-2008 & 68 & 235 & 53.5 & 11.0 & 16.0 & 20.0 & 25.0 & 31.0 \\
\hline 01561000 & $1930-1958$ & 28 & 45.3 & 6.1 & .4 & 1.5 & 2.6 & 3.6 & 4.7 \\
\hline 01562000 & 1912-2008 & 96 & 927 & 281 & 68.0 & 98.0 & 118 & 140 & 163 \\
\hline 01562500 & 1930-1957 & 27 & 95.0 & 13.8 & 1.6 & 3.3 & 4.9 & 6.6 & 8.9 \\
\hline 01563200 & ${ }^{2} 1973-2008$ & 35 & 1,210 & 375 & 111 & 171 & 207 & 223 & 239 \\
\hline 01563200 & ${ }^{3} 1947-1972$ & 25 & 1,080 & 194 & 18.1 & 68.0 & 115 & 142 & 176 \\
\hline 01563500 & ${ }^{2} 1973-2008$ & 35 & 2,750 & 1,240 & 428 & 503 & 562 & 630 & 728 \\
\hline 01563500 & ${ }^{3} 1938-1972$ & 34 & 2,390 & 876 & 227 & 327 & 395 & 454 & 529 \\
\hline 01564500 & 1939-2008 & 69 & 246 & 37.9 & 5.4 & 9.8 & 14.0 & 19.0 & 24.0 \\
\hline
\end{tabular}


Table 5. Selected mean-flow and flow-duration statistics for streamgage locations in and near Pennsylvania.-Continued [ $\mathrm{ft}$ /s; cubic feet per second; --, statistic not computed; <, less than]

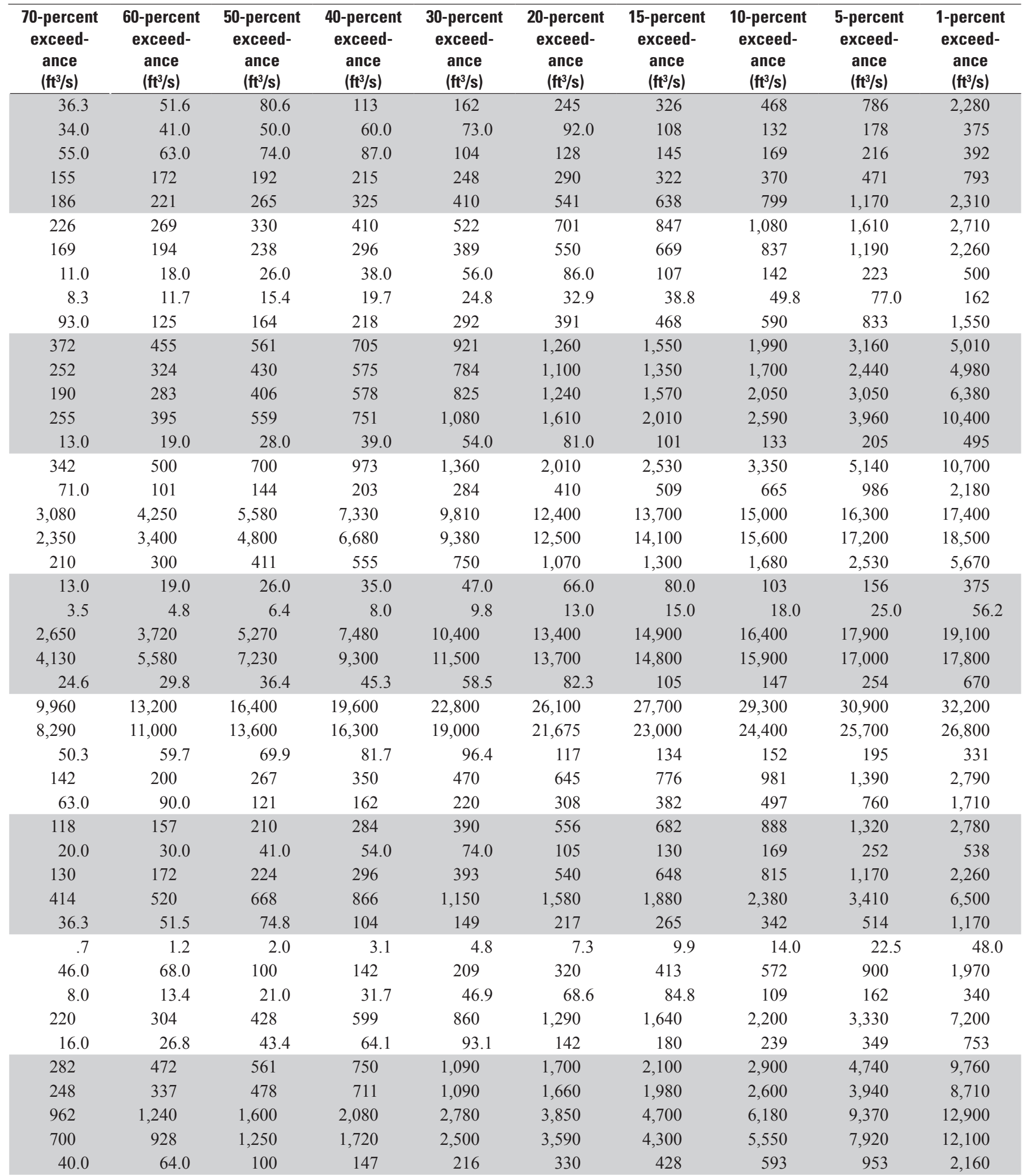


Table 5. Selected mean-flow and flow-duration statistics for streamgage locations in and near Pennsylvania.-Continued

$\left[\mathrm{ft}^{2} / \mathrm{s}\right.$; cubic feet per second; --, statistic not computed; <, less than]

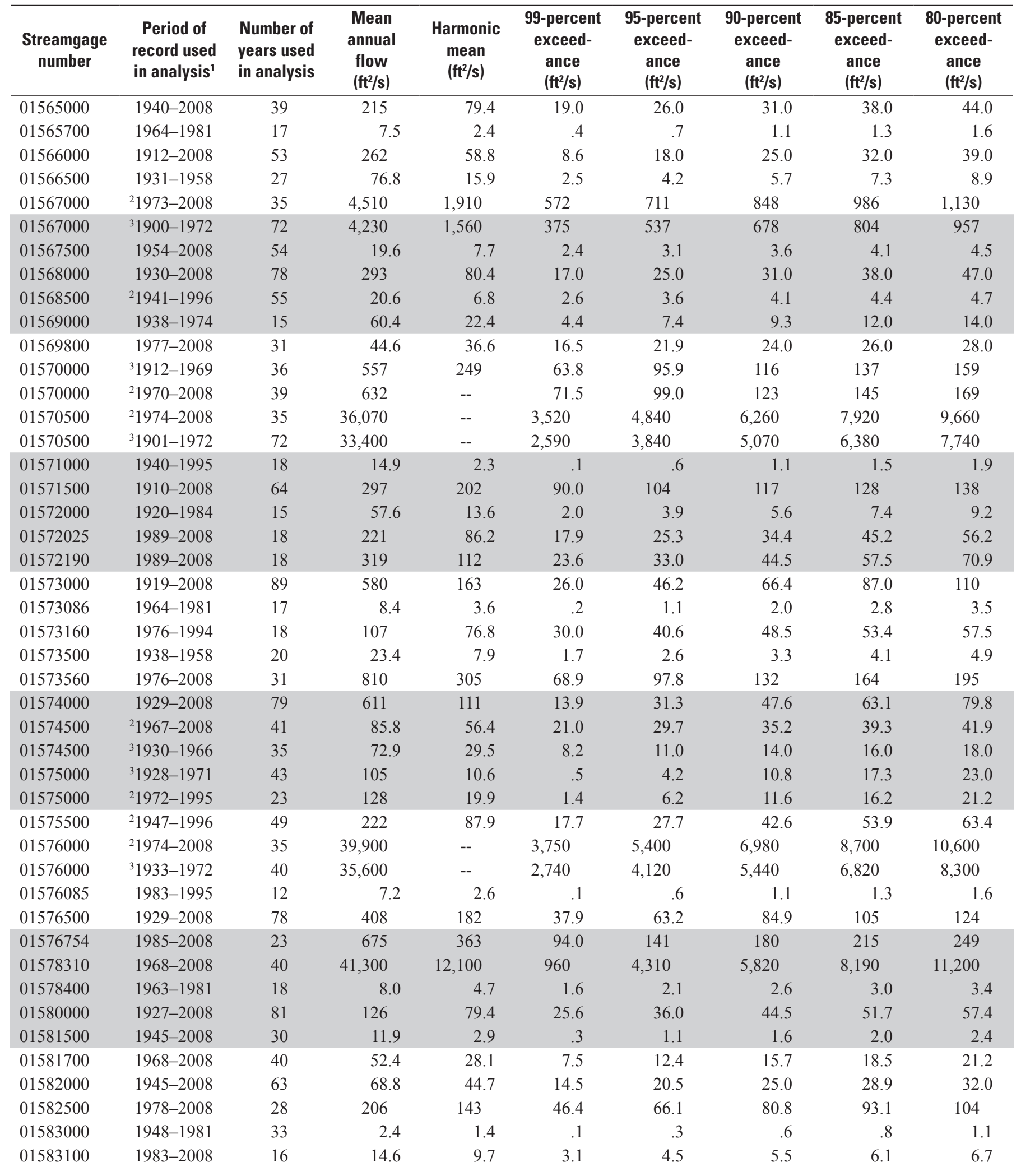


Table 5. Selected mean-flow and flow-duration statistics for streamgage locations in and near Pennsylvania.—Continued $[\mathrm{ft} / \mathrm{s}$; cubic feet per second; --, statistic not computed; <, less than]

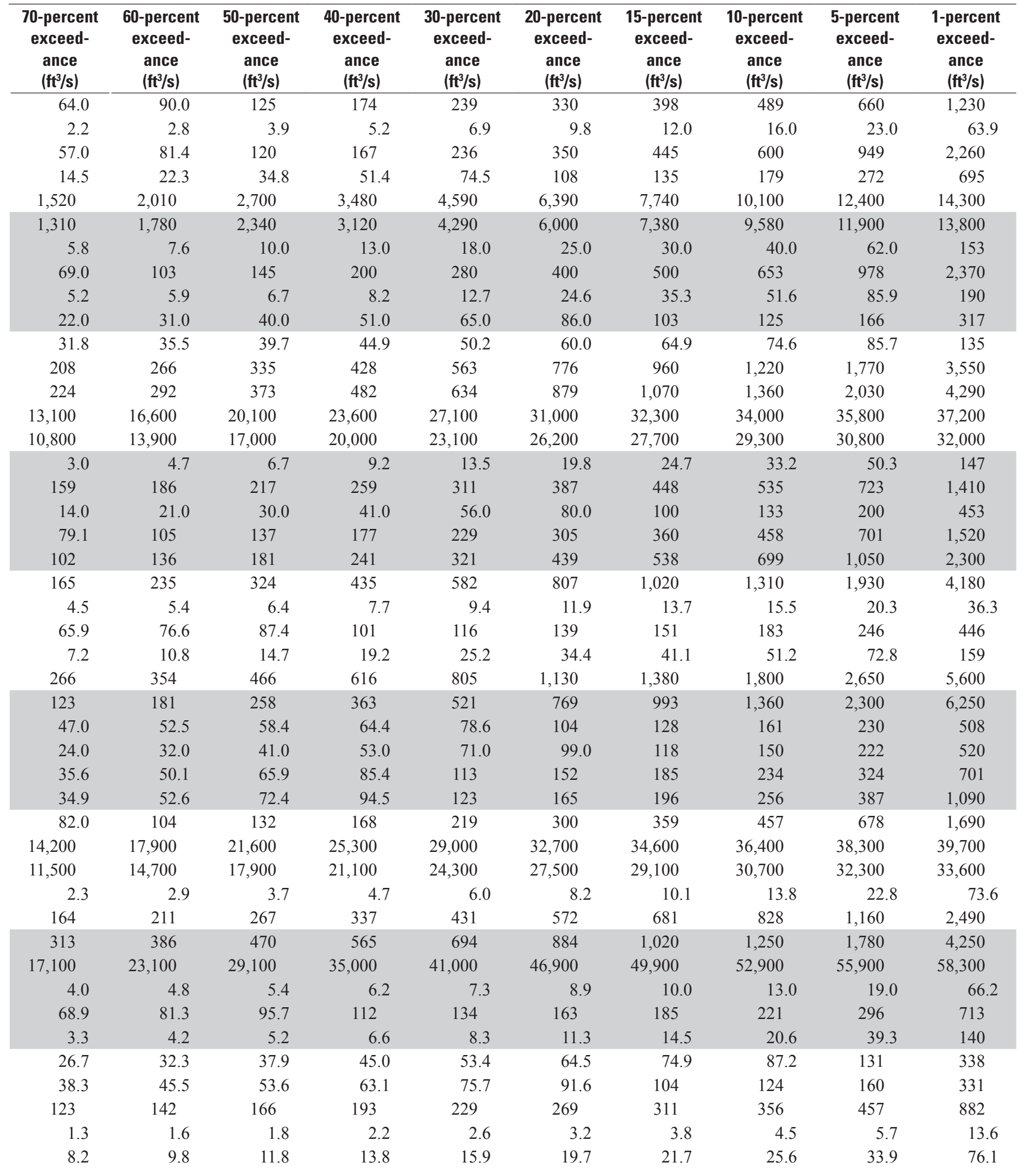


Table 5. Selected mean-flow and flow-duration statistics for streamgage locations in and near Pennsylvania.—Continued

$\left[\mathrm{ft}^{2} / \mathrm{s}\right.$; cubic feet per second; --, statistic not computed; <, less than]

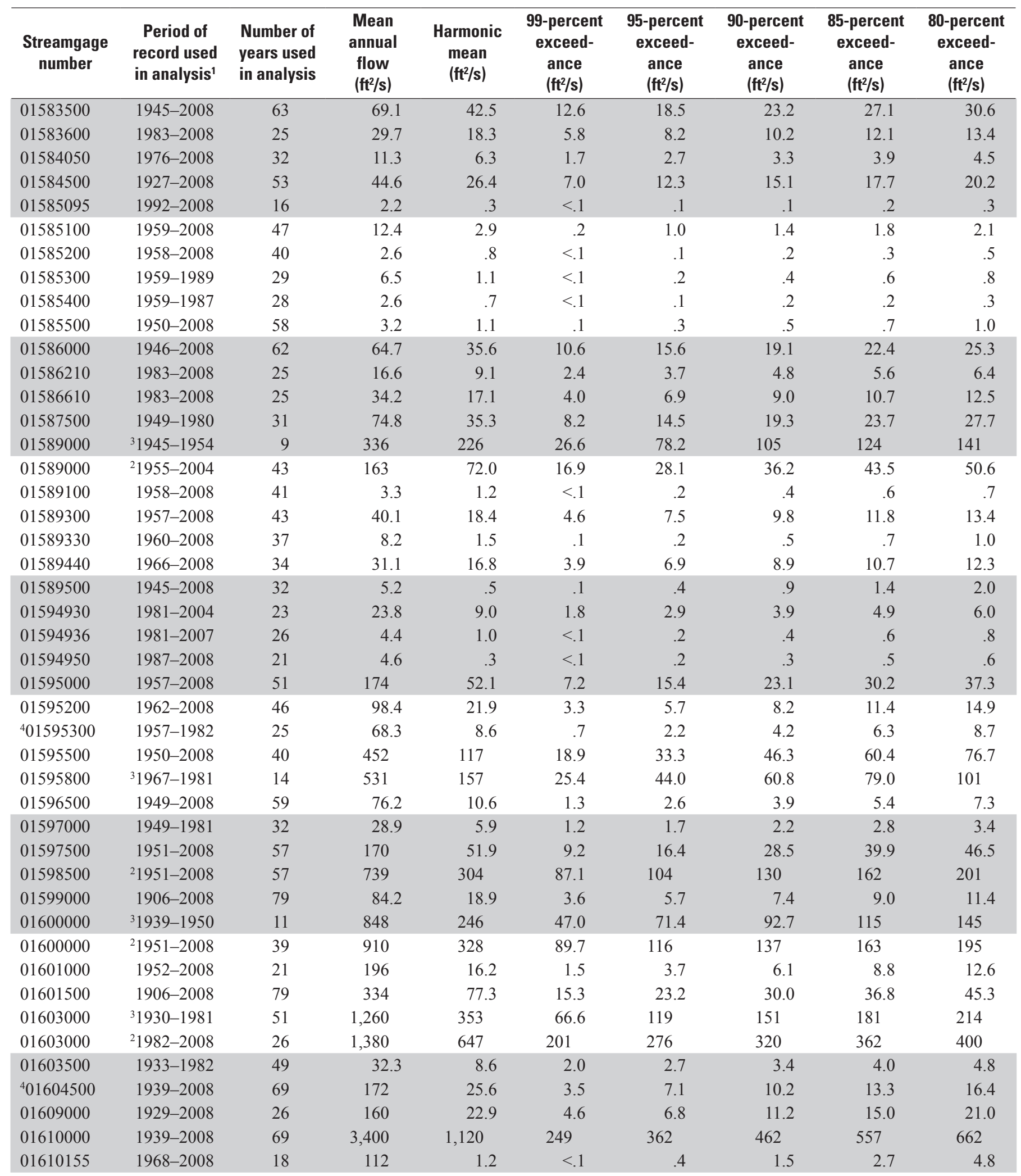


Table 5. Selected mean-flow and flow-duration statistics for streamgage locations in and near Pennsylvania.-Continued $[\mathrm{ft} / \mathrm{s}$; cubic feet per second; --, statistic not computed; <, less than]

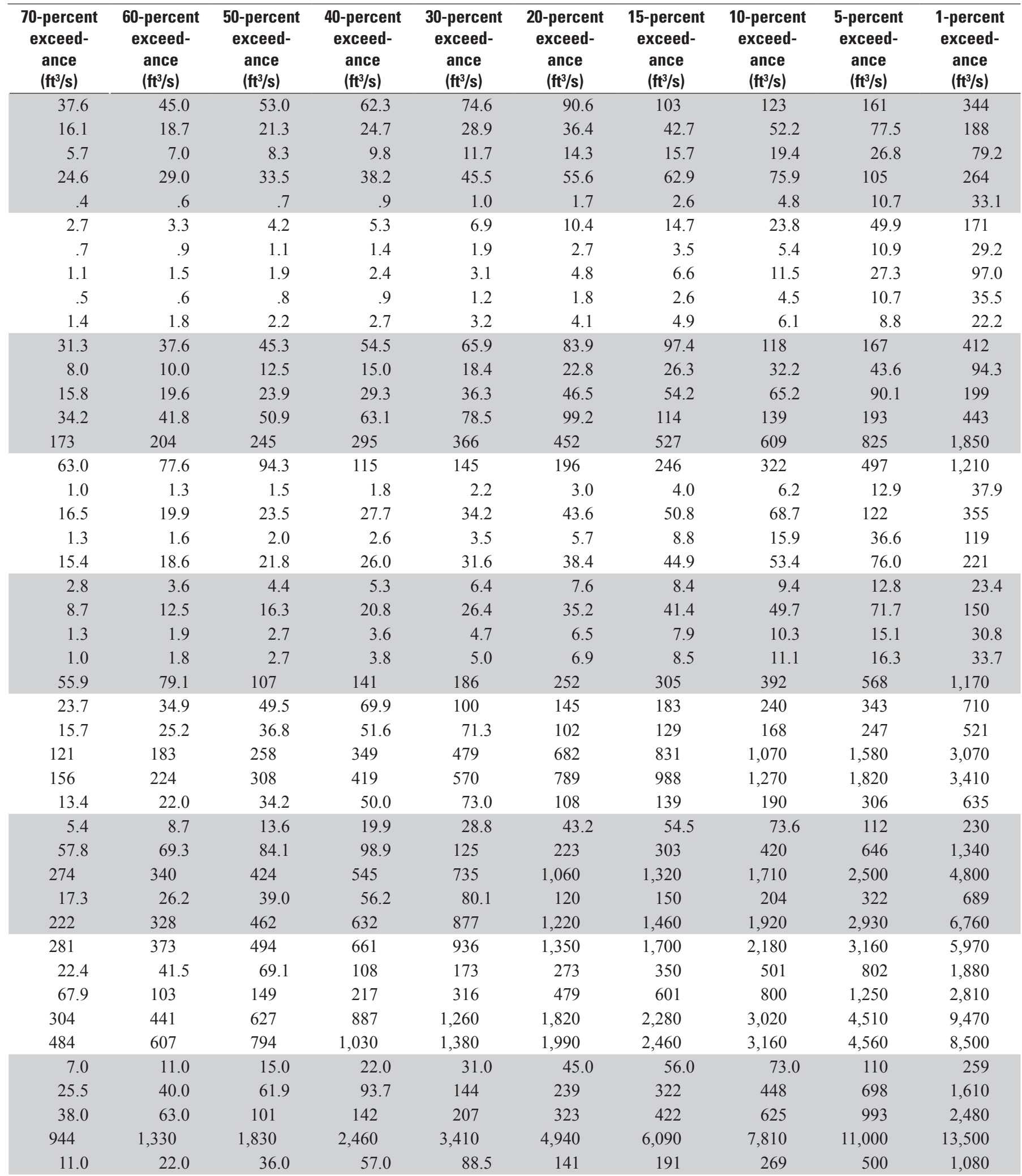


Table 5. Selected mean-flow and flow-duration statistics for streamgage locations in and near Pennsylvania.-Continued

$\left[\mathrm{ft}^{2} / \mathrm{s}\right.$; cubic feet per second; --, statistic not computed; <, less than]

\begin{tabular}{|c|c|c|c|c|c|c|c|c|c|}
\hline $\begin{array}{c}\text { Streamgage } \\
\text { number }\end{array}$ & $\begin{array}{l}\text { Period of } \\
\text { record used } \\
\text { in analysis }\end{array}$ & $\begin{array}{l}\text { Number of } \\
\text { years used } \\
\text { in analysis }\end{array}$ & $\begin{array}{c}\text { Mean } \\
\text { annual } \\
\text { flow } \\
\left(\mathrm{ft}^{2} / \mathrm{s}\right)\end{array}$ & $\begin{array}{c}\text { Harmonic } \\
\text { mean } \\
\left(\mathrm{ft}^{2} / \mathrm{s}\right)\end{array}$ & $\begin{array}{l}\text { 99-percent } \\
\text { exceed- } \\
\text { ance } \\
\left(\mathrm{ft}^{2} / \mathrm{s}\right)\end{array}$ & $\begin{array}{l}\text { 95-percent } \\
\text { exceed- } \\
\text { ance } \\
\left(\mathrm{ft}^{2} / \mathrm{s}\right)\end{array}$ & $\begin{array}{l}\text { 90-percent } \\
\text { exceed- } \\
\text { ance } \\
\left(\mathrm{ft}^{2} / \mathrm{s}\right)\end{array}$ & $\begin{array}{l}\text { 85-percent } \\
\text { exceed- } \\
\text { ance } \\
\left(\mathrm{ft}^{2} / \mathrm{s}\right)\end{array}$ & $\begin{array}{c}\text { 80-percent } \\
\text { exceed- } \\
\text { ance } \\
\left(\mathrm{ft}^{2} / \mathrm{s}\right)\end{array}$ \\
\hline 01613000 & $1933-2008$ & 75 & 4,200 & 1,350 & 304 & 440 & 554 & 668 & 787 \\
\hline 01613050 & $1966-2008$ & 41 & 13.3 & .5 & 0 & .1 & .3 & .6 & .9 \\
\hline 01614500 & 1929-2008 & 79 & 606 & 231 & 56.0 & 84.0 & 103 & 120 & 140 \\
\hline${ }^{4} 01616500$ & $1948-2008$ & 60 & 246 & 119 & 36.4 & 49.3 & 58.0 & 66.5 & 75.1 \\
\hline 01617000 & 1949-2008 & 26 & 10.9 & 4.6 & 1.1 & 1.7 & 2.3 & 2.6 & 3.0 \\
\hline 01617800 & 1964-2008 & 44 & 12.1 & 4.4 & .5 & 1.6 & 2.7 & 3.4 & 4.1 \\
\hline 01618000 & 1929-2004 & 69 & 6,120 & 2,080 & 448 & 686 & 887 & 1,070 & 1,290 \\
\hline 01639000 & $1943-2008$ & 65 & 211 & 16.3 & 1.8 & 4.9 & 7.9 & 11.0 & 16.0 \\
\hline 01639140 & 1991-2001 & 10 & 37.0 & 2.6 & .1 & .6 & 1.2 & 1.9 & 2.8 \\
\hline 01639500 & $1948-2008$ & 60 & 115 & 42.8 & 10.7 & 18.2 & 23.6 & 28.4 & 32.8 \\
\hline 01640500 & 1932-1984 & 52 & 9.5 & 1.9 & .1 & .4 & .7 & 1.1 & 1.4 \\
\hline 01640965 & 1982-1994 & 12 & 3.4 & .4 & $<.1$ & .1 & .3 & .4 & .5 \\
\hline 01640970 & 1982-1991 & 9 & 6.3 & 1.3 & .1 & .2 & .5 & .7 & .9 \\
\hline 01641000 & ${ }^{3} 1951-1968$ & 17 & 22.8 & -- & 1.2 & 1.9 & 2.3 & 2.9 & 3.6 \\
\hline 01641000 & ${ }^{2} 1970-1991$ & 21 & 29.4 & -- & 2.7 & 3.7 & 4.5 & 5.2 & 6.1 \\
\hline 01641500 & 1948-1984 & 36 & 11.8 & 4.2 & 1.0 & 1.4 & 1.7 & 2.0 & 2.4 \\
\hline 01642500 & ${ }^{3} 1933-1970$ & 37 & 77.5 & -- & 8.5 & 13.3 & 17.0 & 20.0 & 23.3 \\
\hline 01642500 & ${ }^{2} 1972-1982$ & 10 & 109 & -- & 12.0 & 17.1 & 21.5 & 25.9 & 30.6 \\
\hline 03010655 & $1975-2008$ & 33 & 158 & 40.2 & 6.2 & 10.0 & 15.0 & 20.0 & 27.0 \\
\hline 03011020 & 1904-2008 & 104 & 2,790 & 778 & 132 & 213 & 292 & 386 & 495 \\
\hline 03011800 & 1966-2008 & 42 & 78.7 & 28.5 & 5.1 & 8.4 & 12.0 & 15.0 & 19.0 \\
\hline 03012550 & ${ }^{3} 1936-1965$ & 30 & 3,710 & -- & 204 & 268 & 341 & 433 & 555 \\
\hline 03012550 & ${ }^{2} 1966-1991$ & 26 & 3,990 & -- & 364 & 635 & 740 & 898 & 1,150 \\
\hline 03013000 & 1939-1993 & 54 & 539 & 153 & 34.0 & 44.0 & 55.0 & 70.0 & 87.0 \\
\hline 03014500 & 1935-2008 & 73 & 366 & 88.2 & 10.0 & 23.8 & 38.8 & 51.8 & 60.6 \\
\hline 03015000 & ${ }^{3} 1940-1949$ & 9 & 1,470 & 448 & 79.8 & 117 & 157 & 206 & 254 \\
\hline 03015000 & ${ }^{2} 1950-2008$ & 58 & 1,560 & 457 & 82.6 & 128 & 170 & 211 & 260 \\
\hline 03015280 & 1963-1978 & 15 & 23.2 & 6.2 & 1.1 & 1.7 & 2.3 & 3.0 & 3.7 \\
\hline 03015500 & 1910-2008 & 98 & 600 & 176 & 36.0 & 52.0 & 68.0 & 86.0 & 107 \\
\hline 03016000 & ${ }^{3} 1942-1965$ & 23 & 6,340 & 1,740 & 369 & 497 & 614 & 764 & 987 \\
\hline 03016000 & ${ }^{2} 1960-2008$ & 45 & 6,730 & 2,920 & 478 & 991 & 1,470 & 1,770 & 2,050 \\
\hline 03017500 & 1938-1979 & 41 & 428 & 115 & 20.2 & 30.5 & 43.2 & 58.4 & 75.6 \\
\hline 03020000 & 1941-1991 & 50 & 893 & 116 & 32.1 & 58.6 & 84.8 & 109 & 137 \\
\hline
\end{tabular}


Table 5. Selected mean-flow and flow-duration statistics for streamgage locations in and near Pennsylvania.—Continued $\left[\mathrm{ft}^{3} / \mathrm{s}\right.$; cubic feet per second; --, statistic not computed; <, less than]

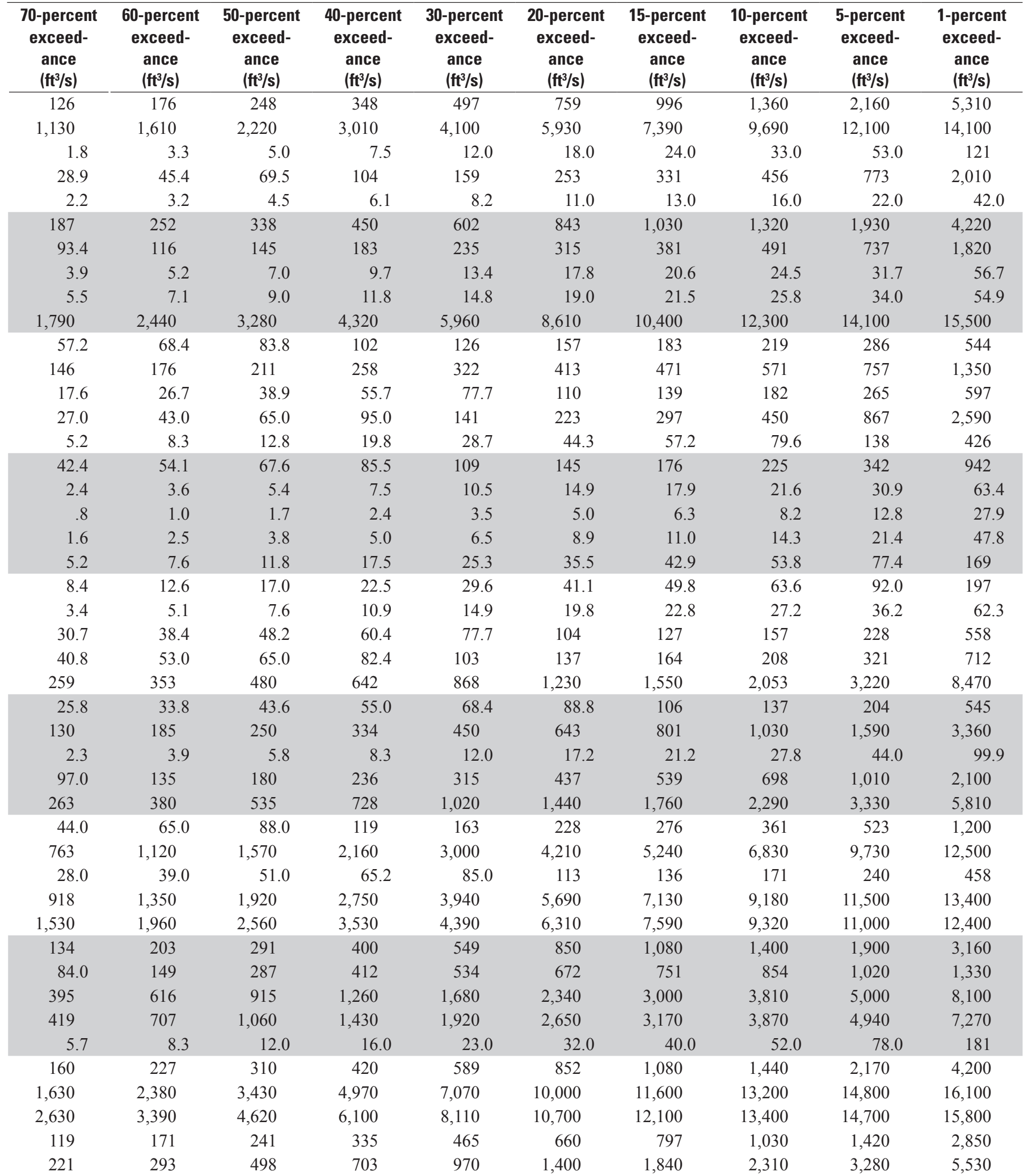


Table 5. Selected mean-flow and flow-duration statistics for streamgage locations in and near Pennsylvania.-Continued [ $\mathrm{ft}^{2} / \mathrm{s}$; cubic feet per second; --, statistic not computed; $<$, less than]

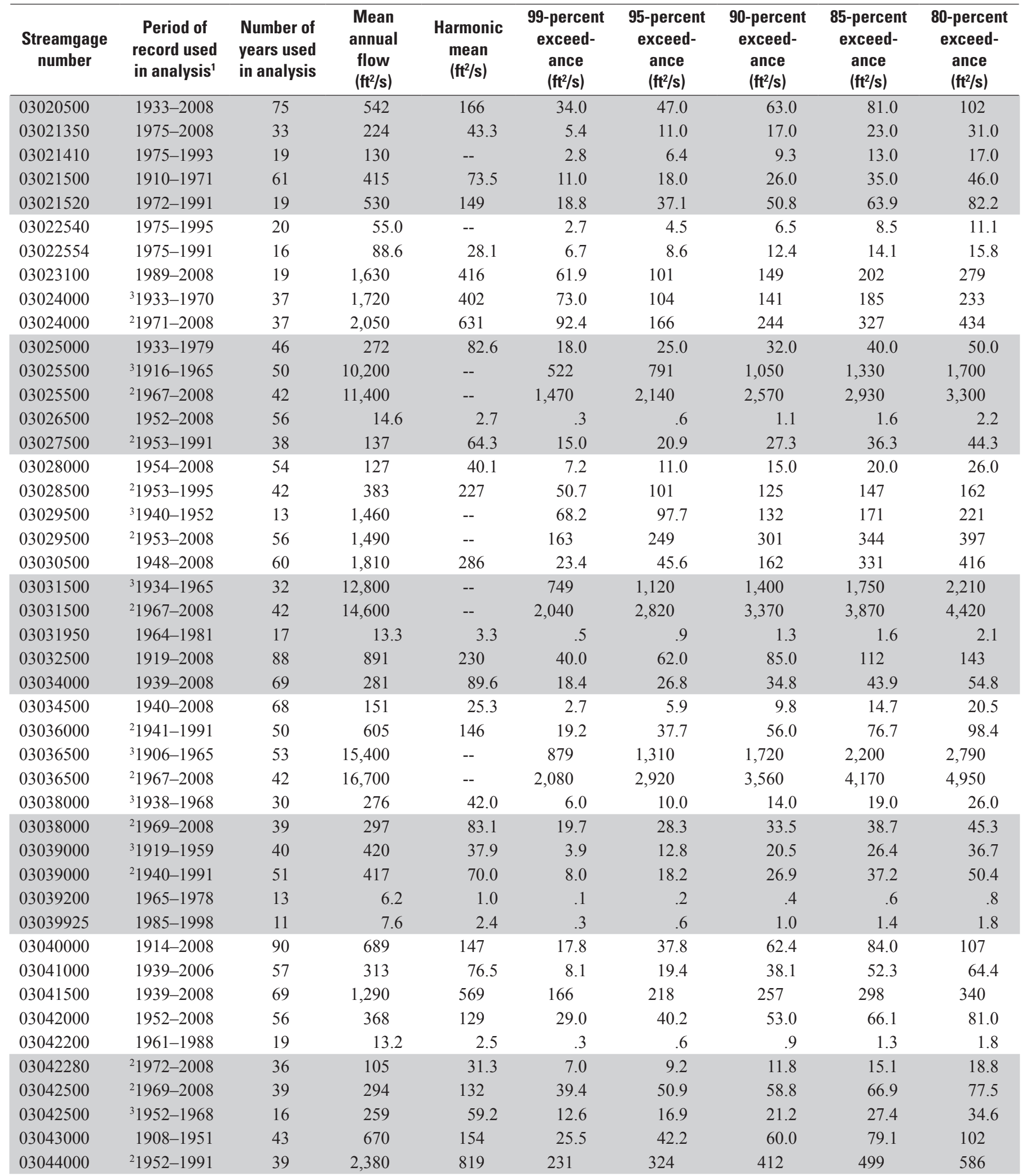


Table 5. Selected mean-flow and flow-duration statistics for streamgage locations in and near Pennsylvania.—Continued [ $\mathrm{ft}^{3} / \mathrm{s}$; cubic feet per second; --, statistic not computed; <, less than]

\begin{tabular}{|c|c|c|c|c|c|c|c|c|c|}
\hline $\begin{array}{l}\text { 70-percent } \\
\text { exceed- } \\
\text { ance } \\
\left(\mathrm{ft}^{3} / \mathrm{s}\right)\end{array}$ & $\begin{array}{c}\text { 60-percent } \\
\text { exceed- } \\
\text { ance } \\
\left(\mathrm{ft}^{3} / \mathrm{s}\right)\end{array}$ & $\begin{array}{c}\text { 50-percent } \\
\text { exceed- } \\
\text { ance } \\
\left(\mathrm{ft}^{3} / \mathrm{s}\right)\end{array}$ & $\begin{array}{c}\text { 40-percent } \\
\text { exceed- } \\
\text { ance } \\
\left(\mathrm{ft}^{3} / \mathrm{s}\right)\end{array}$ & $\begin{array}{c}\text { 30-percent } \\
\text { exceed- } \\
\text { ance } \\
\left(\mathrm{ft}^{3} / \mathrm{s}\right)\end{array}$ & $\begin{array}{c}\text { 20-percent } \\
\text { exceed- } \\
\text { ance } \\
\left(\mathrm{ft}^{3} / \mathrm{s}\right)\end{array}$ & $\begin{array}{c}\text { 15-percent } \\
\text { exceed- } \\
\text { ance } \\
\left(\mathrm{ft}^{3} / \mathrm{s}\right)\end{array}$ & $\begin{array}{c}\text { 10-percent } \\
\text { exceed- } \\
\text { ance } \\
\left(\mathrm{ft}^{3} / \mathrm{s}\right)\end{array}$ & $\begin{array}{c}\text { 5-percent } \\
\text { exceed- } \\
\text { ance } \\
\left(\mathrm{ft}^{3} / \mathrm{s}\right)\end{array}$ & $\begin{array}{c}\text { 1-percent } \\
\text { exceed- } \\
\text { ance } \\
\left(\mathrm{ft}^{3} / \mathrm{s}\right)\end{array}$ \\
\hline 52.0 & 78.0 & 104 & 140 & 192 & 292 & 375 & 530 & 875 & 1,940 \\
\hline 28.6 & 43.5 & 59.6 & 80.6 & 113 & 180 & 234 & 321 & 504 & 1,090 \\
\hline 77.0 & 125 & 188 & 270 & 390 & 585 & 752 & 1,020 & 1,580 & 3,310 \\
\hline 17.1 & 23.2 & 30.3 & 38.8 & 52.2 & 75.2 & 93.5 & 127 & 192 & 439 \\
\hline 22.8 & 37.0 & 52.9 & 66.8 & 101 & 134 & 157 & 231 & 319 & 546 \\
\hline 484 & 767 & 1,160 & 1,600 & 2,060 & 2,620 & 3,080 & 3,760 & 5,080 & 8,330 \\
\hline 386 & 600 & 898 & 1,270 & 1,840 & 2,690 & 3,360 & 4,360 & 6,180 & 11,000 \\
\hline 699 & 1025 & 1,420 & 1,940 & 2,530 & 3,280 & 3,880 & 4,690 & 6,160 & 9,940 \\
\hline 60.5 & 79.6 & 110 & 138 & 168 & 201 & 230 & 258 & 365 & 591 \\
\hline 39.0 & 56.0 & 76.0 & 100 & 133 & 185 & 224 & 290 & 418 & 816 \\
\hline 191 & 224 & 261 & 319 & 405 & 542 & 648 & 799 & 1,070 & 1,860 \\
\hline 360 & 549 & 789 & 1,140 & 1,660 & 2,340 & 2,820 & 3,450 & 4,950 & 9,750 \\
\hline 529 & 708 & 943 & 1,240 & 1,640 & 2,220 & 2,650 & 3,270 & 4,640 & 8,860 \\
\hline 615 & 867 & 1,160 & 1,570 & 2,070 & 2,790 & 3,250 & 4,130 & 5,540 & 10,200 \\
\hline 3,400 & 4,940 & 7,020 & 10,100 & 13,400 & 16,600 & 18,300 & 19,900 & 21,600 & 22,900 \\
\hline 5,930 & 7,980 & 10,500 & 12,900 & 15,400 & 17,900 & 19,100 & 20,400 & 21,600 & 22,600 \\
\hline 3.4 & 4.9 & 6.9 & 9.4 & 13.0 & 18.0 & 22.0 & 29.0 & 47.0 & 109 \\
\hline 229 & 335 & 470 & 660 & 920 & 1,330 & 1,630 & 2,130 & 3,090 & 5,990 \\
\hline 64.6 & 97.0 & 141 & 201 & 284 & 417 & 523 & 693 & 1,060 & 2,430 \\
\hline 64.6 & 105 & 168 & 242 & 385 & 644 & 787 & 1,070 & 1,760 & 3,670 \\
\hline 81.3 & 120 & 186 & 252 & 430 & 690 & 810 & 1,090 & 1,700 & 2,980 \\
\hline 1.4 & 2.4 & 3.3 & 4.4 & 6.0 & 8.5 & 11.0 & 14.0 & 23.0 & 52.0 \\
\hline 2.5 & 3.7 & 4.9 & 6.3 & 8.4 & 11.0 & 13.0 & 17.0 & 24.0 & 44.2 \\
\hline 166 & 243 & 345 & 490 & 694 & 1,020 & 1,280 & 1,690 & 2,470 & 5,140 \\
\hline 89.6 & 124 & 170 & 233 & 318 & 444 & 547 & 708 & 1,050 & 2,330 \\
\hline 438 & 568 & 746 & 1,000 & 1,350 & 1,880 & 2,300 & 2,910 & 4,090 & 8,070 \\
\hline 114 & 156 & 213 & 285 & 387 & 530 & 636 & 803 & 1,210 & 2,510 \\
\hline 3.1 & 4.6 & 6.5 & 9.0 & 12.0 & 18.0 & 23.0 & 30.0 & 46.0 & 104 \\
\hline 28.6 & 41.7 & 60.4 & 84.9 & 116 & 160 & 191 & 242 & 332 & 672 \\
\hline 104 & 138 & 180 & 236 & 309 & 418 & 500 & 616 & 907 & 1,830 \\
\hline 54.1 & 81.9 & 121 & 179 & 256 & 384 & 483 & 642 & 964 & 2,000 \\
\hline 160 & 237 & 336 & 472 & 676 & 986 & 1,230 & 1,610 & 2,400 & 5,150 \\
\hline 798 & 1,090 & 1,440 & 1,870 & 2,490 & 3,510 & 4,260 & 5,460 & 7,860 & 11,900 \\
\hline
\end{tabular}


Table 5. Selected mean-flow and flow-duration statistics for streamgage locations in and near Pennsylvania.-Continued

$\left[\mathrm{ft}^{2} / \mathrm{s}\right.$; cubic feet per second; --, statistic not computed; <, less than]

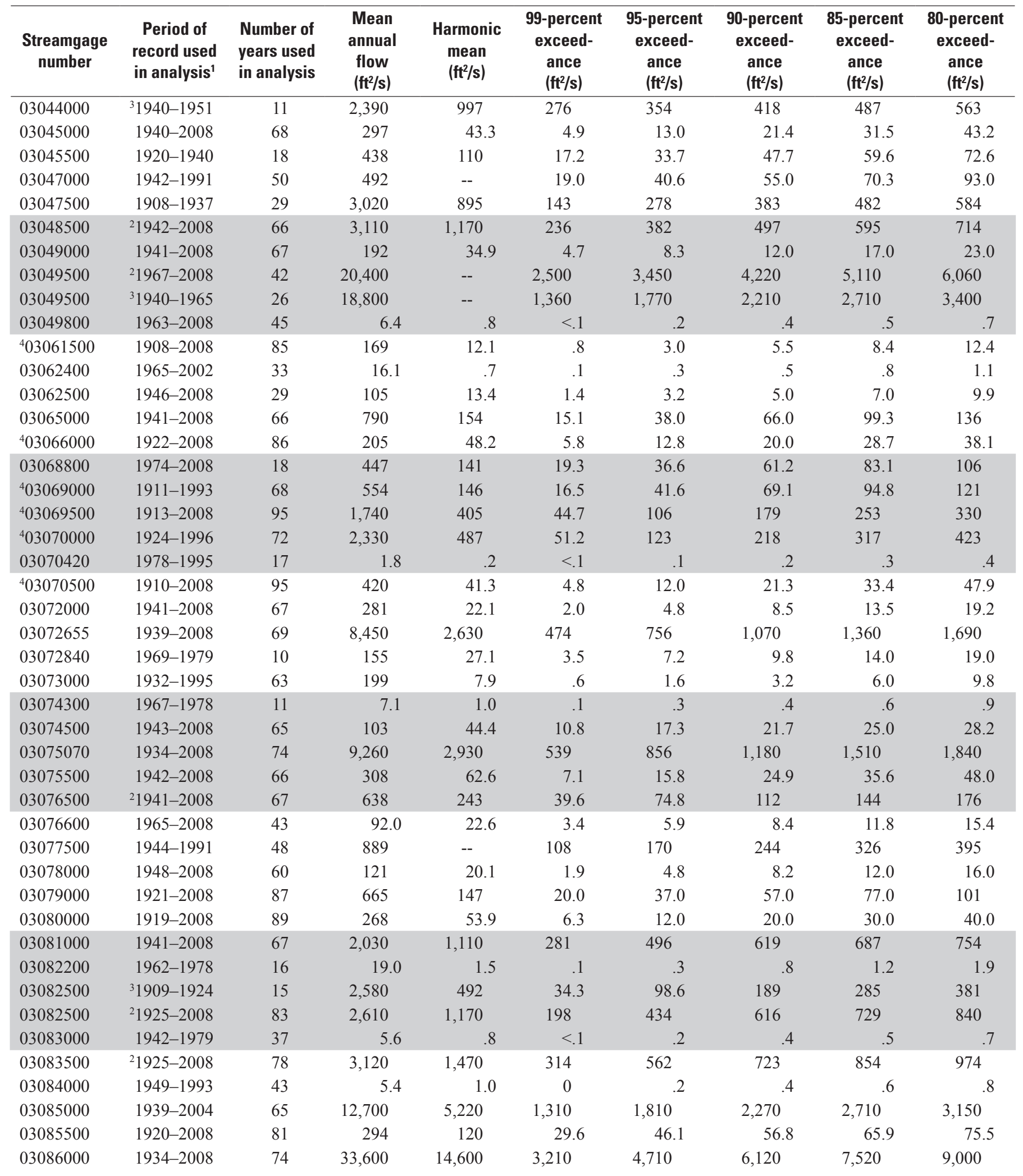


Table 5. Selected mean-flow and flow-duration statistics for streamgage locations in and near Pennsylvania.-Continued

$\left[\mathrm{ft}^{3} / \mathrm{s}\right.$; cubic feet per second; --, statistic not computed; <, less than]

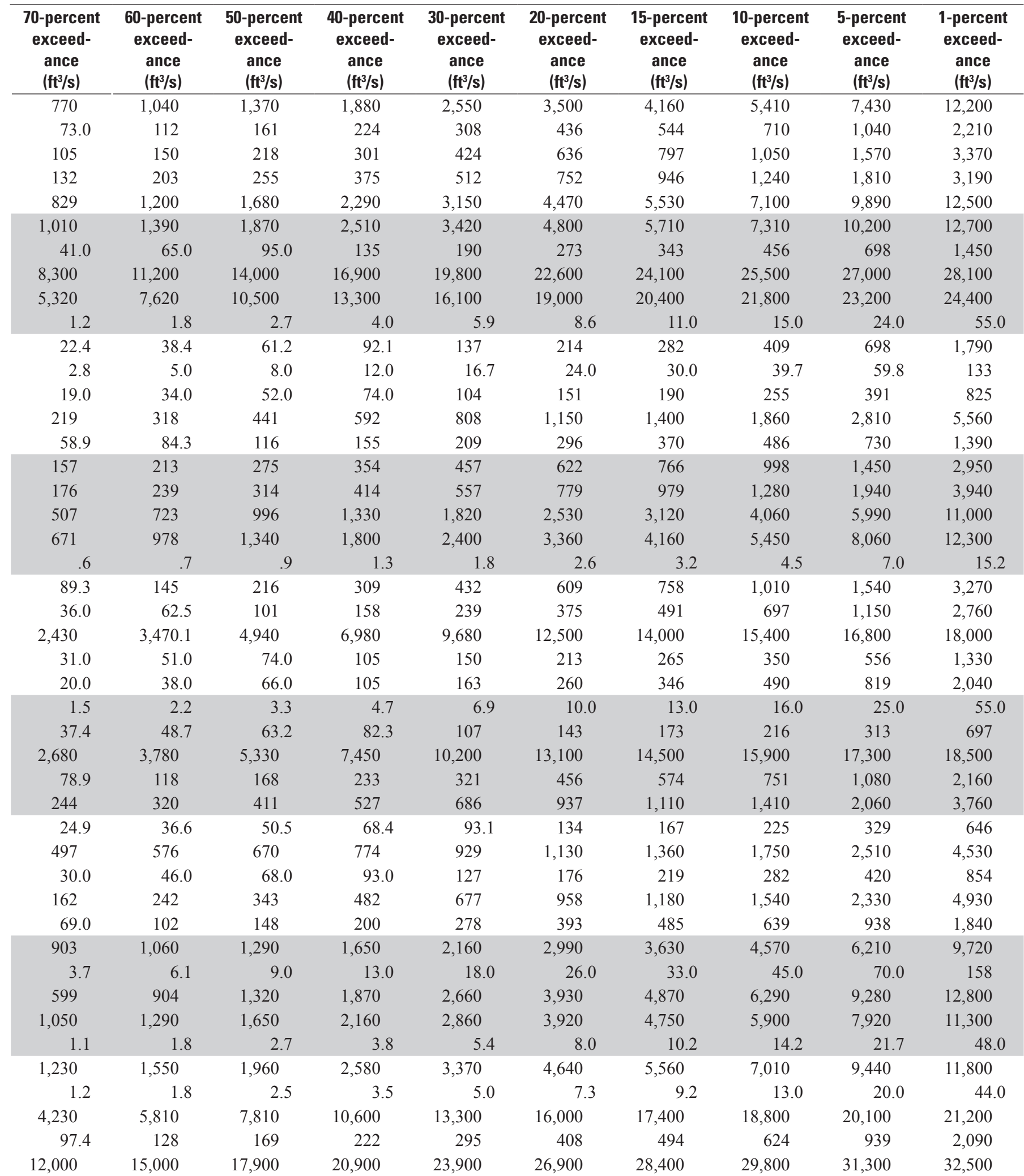


Table 5. Selected mean-flow and flow-duration statistics for streamgage locations in and near Pennsylvania.-Continued

[ $\mathrm{ft}^{2} / \mathrm{s}$; cubic feet per second; --, statistic not computed; <, less than]

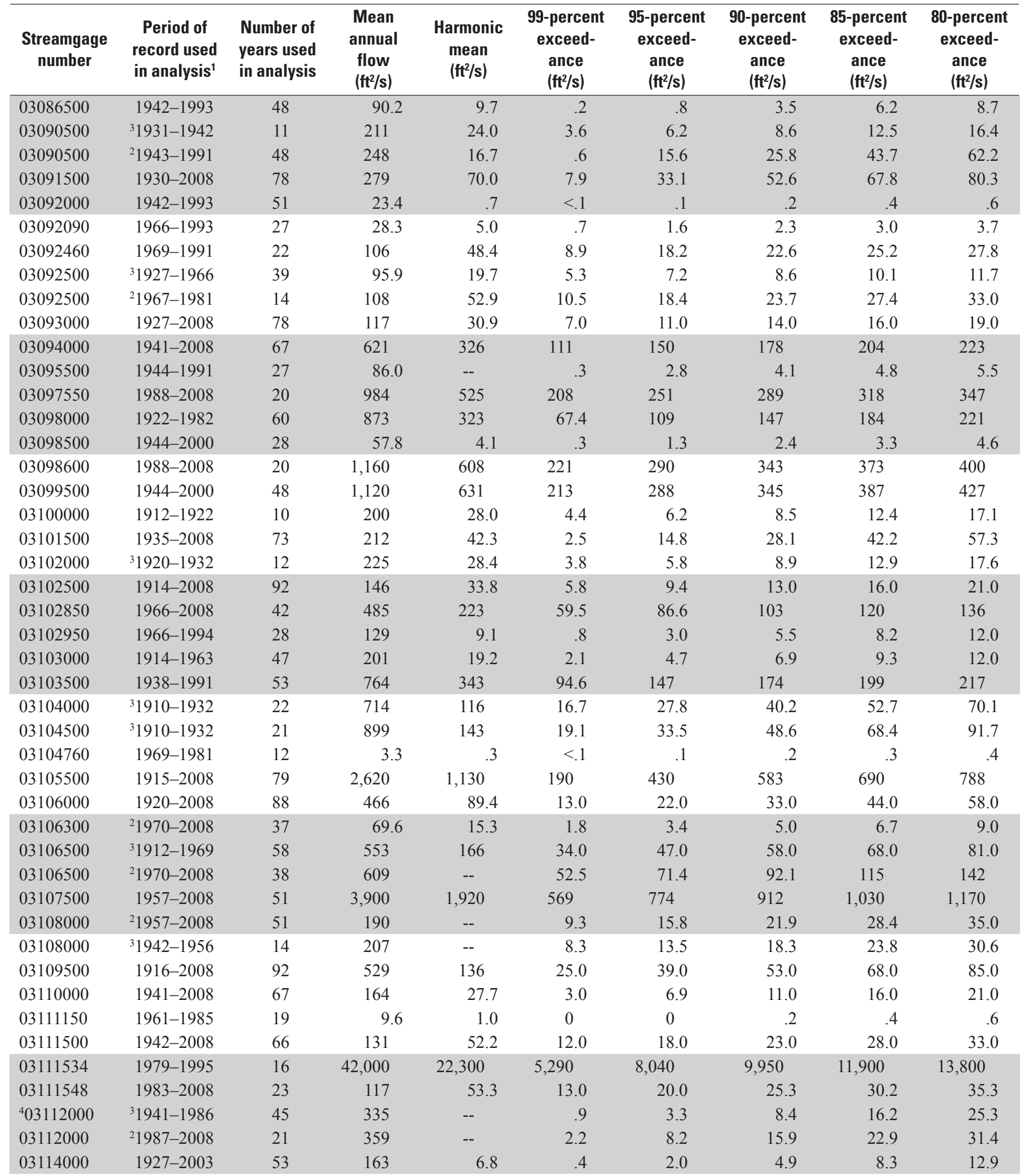


Table 5. Selected mean-flow and flow-duration statistics for streamgage locations in and near Pennsylvania.—Continued [ $\mathrm{ft} 3 / \mathrm{s}$; cubic feet per second; --, statistic not computed; $<$, less than]

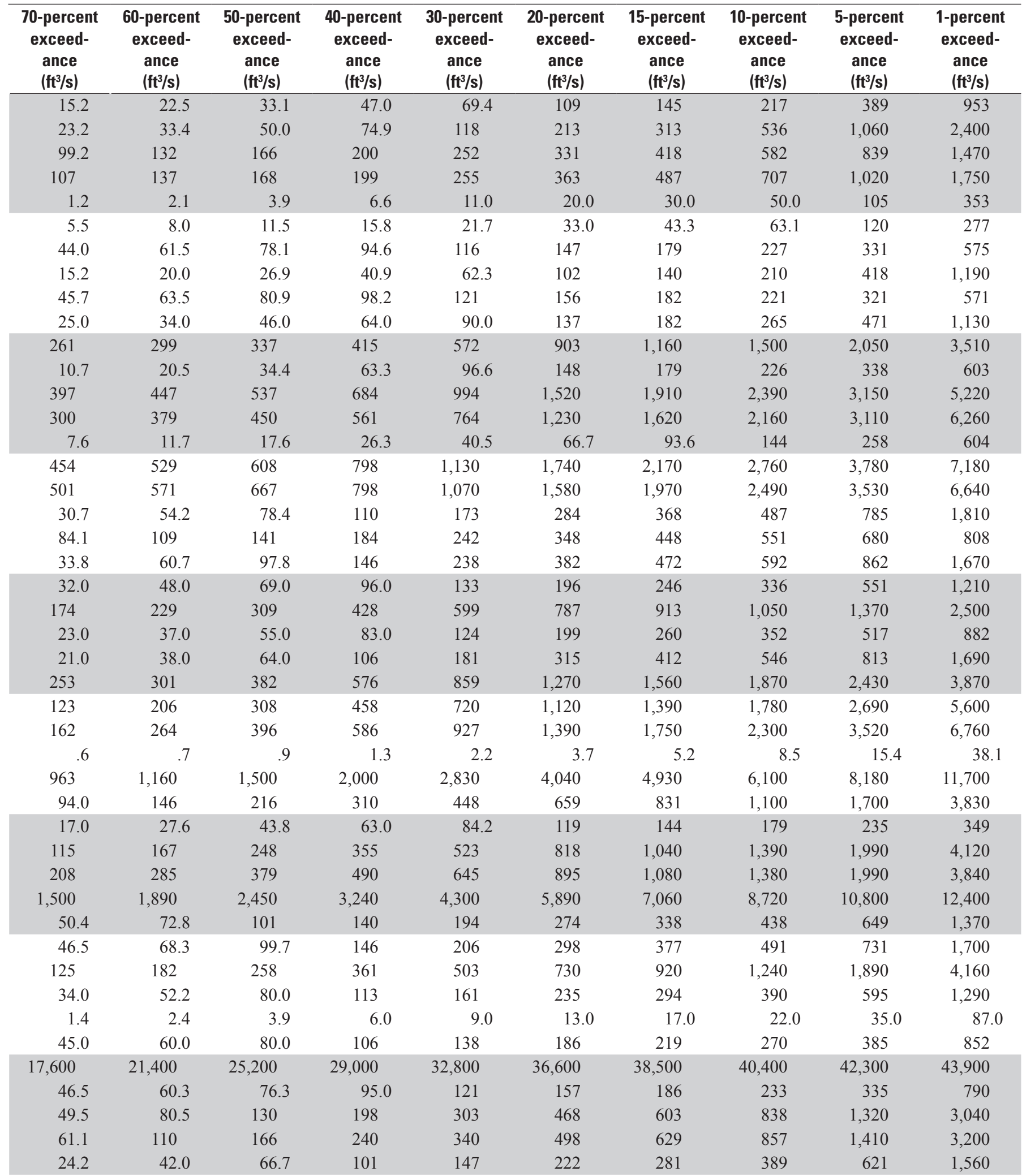


Table 5. Selected mean-flow and flow-duration statistics for streamgage locations in and near Pennsylvania.—Continued $\left[\mathrm{ft}^{2} / \mathrm{s}\right.$; cubic feet per second; --, statistic not computed; <, less than]

\begin{tabular}{|c|c|c|c|c|c|c|c|c|c|}
\hline $\begin{array}{c}\text { Streamgage } \\
\text { number }\end{array}$ & $\begin{array}{c}\text { Period of } \\
\text { record used } \\
\text { in analysis }\end{array}$ & $\begin{array}{l}\text { Number of } \\
\text { years used } \\
\text { in analysis }\end{array}$ & $\begin{array}{c}\text { Mean } \\
\text { annual } \\
\text { flow } \\
\left(\mathrm{ft}^{2} / \mathbf{s}\right)\end{array}$ & $\begin{array}{c}\text { Harmonic } \\
\text { mean } \\
\left(\mathrm{ft}^{2} / \mathrm{s}\right)\end{array}$ & $\begin{array}{l}\text { 99-percent } \\
\text { exceed- } \\
\text { ance } \\
\left(\mathrm{ft}^{2} / \mathrm{s}\right)\end{array}$ & $\begin{array}{l}\text { 95-percent } \\
\text { exceed- } \\
\text { ance } \\
\left(\mathrm{ft}^{2} / \mathrm{s}\right)\end{array}$ & $\begin{array}{l}\text { 90-percent } \\
\text { exceed- } \\
\text { ance } \\
\left(\mathrm{ft}^{2} / \mathrm{s}\right)\end{array}$ & $\begin{array}{c}\text { 85-percent } \\
\text { exceed- } \\
\text { ance } \\
\left(\mathrm{ft}^{2} / \mathrm{s}\right)\end{array}$ & $\begin{array}{c}\text { 80-percent } \\
\text { exceed- } \\
\text { ance } \\
\left(\mathrm{ft}^{2} / \mathrm{s}\right)\end{array}$ \\
\hline 04212500 & 1925-1979 & 46 & 154 & 2.0 & 0 & .1 & .5 & 1.2 & 2.5 \\
\hline 04213000 & $1923-2008$ & 71 & 277 & 28.3 & 3.1 & 6.8 & 11.0 & 15.0 & 21.0 \\
\hline 04221000 & $1956-2008$ & 38 & 398 & 108 & 19.9 & 30.1 & 40.8 & 54.0 & 69.0 \\
\hline 04223000 & 1909-2008 & 99 & 1,280 & 349 & 66.3 & 100 & 139 & 177 & 220 \\
\hline 04224775 & $1975-2008$ & 33 & 102 & 32.8 & 7.6 & 10.6 & 13.8 & 17.0 & 20.4 \\
\hline 04227000 & 1959-2008 & 45 & 306 & 98.6 & 22.9 & 32.4 & 41.6 & 49.8 & 60.8 \\
\hline
\end{tabular}

${ }^{1}$ Water year is defined as 12-month period beginning October 1 and ending September 30 . The water year is designated by the calendar year in which it ends.

${ }^{3}$ Period of record for post-regulation period.

${ }^{2}$ Period of record for pre-regulation period.

${ }^{4}$ Low-flow statistics computed from differing period of record can be found in Wiley (2006). 
Table 5. Selected mean-flow and flow-duration statistics for streamgage locations in and near Pennsylvania.—Continued $\left[\mathrm{ft}^{3} / \mathrm{s}\right.$; cubic feet per second; --, statistic not computed; <, less than]

\begin{tabular}{|c|c|c|c|c|c|c|c|c|c|}
\hline $\begin{array}{c}\text { 70-percent } \\
\text { exceed- } \\
\text { ance } \\
\left(\mathrm{ft}^{3} / \mathrm{s}\right)\end{array}$ & $\begin{array}{c}\text { 60-percent } \\
\text { exceed- } \\
\text { ance } \\
\left(\mathrm{ft}^{3} / \mathrm{s}\right)\end{array}$ & $\begin{array}{c}\text { 50-percent } \\
\text { exceed- } \\
\text { ance } \\
\left(\mathrm{ft}^{3} / \mathrm{s}\right)\end{array}$ & $\begin{array}{c}\text { 40-percent } \\
\text { exceed- } \\
\text { ance } \\
\left(\mathrm{ft}^{3} / \mathrm{s}\right)\end{array}$ & $\begin{array}{c}\text { 30-percent } \\
\text { exceed- } \\
\text { ance } \\
\left(\mathrm{ft}^{3} / \mathrm{s}\right)\end{array}$ & $\begin{array}{c}\text { 20-percent } \\
\text { exceed- } \\
\text { ance } \\
\left(\mathrm{ft}^{3} / \mathrm{s}\right)\end{array}$ & $\begin{array}{c}\text { 15-percent } \\
\text { exceed- } \\
\text { ance } \\
\left(\mathrm{ft}^{3} / \mathrm{s}\right)\end{array}$ & $\begin{array}{c}\text { 10-percent } \\
\text { exceed- } \\
\text { ance } \\
\left(\mathrm{ft}^{3} / \mathrm{s}\right)\end{array}$ & $\begin{array}{c}\text { 5-percent } \\
\text { exceed- } \\
\text { ance } \\
\left(\mathrm{ft}^{3} / \mathrm{s}\right)\end{array}$ & $\begin{array}{c}\text { 1-percent } \\
\text { exceed- } \\
\text { ance } \\
\left(\mathrm{ft}^{3} / \mathrm{s}\right)\end{array}$ \\
\hline 7.6 & 20.0 & 39.0 & 64.0 & 103 & 184 & 260 & 400 & 720 & 1,640 \\
\hline 39.0 & 66.0 & 100 & 150 & 221 & 353 & 480 & 700 & 1,190 & 2,610 \\
\hline 105 & 156 & 216 & 292 & 397 & 559 & 692 & 912 & 1,370 & 3,130 \\
\hline 325 & 457 & 626 & 860 & 1,210 & 1,770 & 2,230 & 3,010 & 4,720 & 10,200 \\
\hline 28.8 & 40.8 & 55.2 & 73.6 & 99.8 & 139 & 171 & 225 & 348 & 815 \\
\hline 84.4 & 116 & 157 & 211 & 290 & 426 & 541 & 730 & 1,160 & 2,370 \\
\hline
\end{tabular}


Table 6. Comparison of streamflow statistics for 72 streamgage locations in Pennsylvania to previously reported streamflow statistics.

[7Q10, 7-day, 10-year low flow; 30Q10, 30-day, 10-year; HM, harmonic mean; P95, 95-percent flow-duration exceedance flow; P50, 50-percent flowduration exceedance flow; P5, 5-percent flow-duration exceedance flow; $<$, less than]

\begin{tabular}{lllc}
\hline $\begin{array}{c}\text { Flow } \\
\text { statistic }\end{array}$ & $\begin{array}{c}\text { Conclusion of } \\
\text { Wilcoxon signed- } \\
\text { rank test }\end{array}$ & $\begin{array}{c}\text { Change in } \\
\text { median flow } \\
\text { statistic }\end{array}$ & $\begin{array}{c}\text { P-value from } \\
\text { Wilcoxon } \\
\text { signed-rank test }\end{array}$ \\
\hline 7 Q10 & Not significant & Decrease & 0.081 \\
30Q10 & Significant & Decrease & .009 \\
HM & Significant & Increase & .001 \\
P95 & Not significant & Decrease & .346 \\
P50 & Significant & Increase & $<.001$ \\
P5 & Significant & Increase & $<.001$ \\
\hline
\end{tabular}

${ }^{1}$ Ehlke and Reed, 1999. 
For more information about this publication, contact: U.S. Geological Survey

215 Limekiln Road

New Cumberland, PA 17070

http://pa.water.usgs.gov/ 


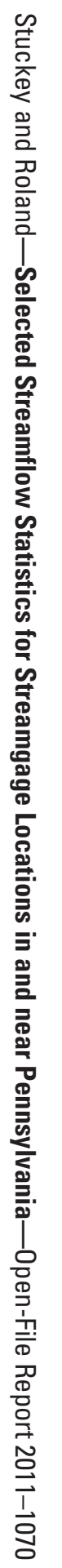

